\title{
ESTUDIO ETNOBOTÁNICO DE ZAPOTITLÁN SALINAS, PUEBLA
}

\author{
Martín Paredes-Flores, Rafael Lira Saade y Patricia D. Dávila Aranda \\ Universidad Nacional Autónoma de México, Facultad de Estudios Superiores \\ Iztacala, Unidad de Biología, Tecnología y Prototipos (UBIPRO), Laboratorio de \\ Recursos Naturales, Avenida de Los Barrios 1, Los Reyes Iztacala, Tlalnepantla, \\ 54090 Estado de México, México \\ rlira@servidor.unam.mx
}

\section{RESUMEN}

En este trabajo se documenta el papel y potencial de los recursos vegetales de Zapotitlán Salinas, Puebla, una comunidad de origen popoloca, ubicada en la porción más árida del Valle de Tehuacán-Cuicatlán. En total, se encontraron 298 especies divididas en 82 familias, y se presenta información etnobotánica de 288 especies pertenecientes a 219 géneros y 79 familias, cuyos usos se ubican en 19 categorías. De ellas, 164 prosperan en las distintas comunidades vegetales naturales de la zona y en algunas de las áreas transformadas, mientras que 124 se encuentran de manera exclusiva en los huertos. Las familias con mayor número de especies útiles fueron Poaceae (35 spp.), Cactaceae (24 spp.) y Asteraceae (23 spp.). En cuanto a las formas de vida, la mayor parte (162 spp.) son las herbáceas, seguida de los arbustos (86 spp.) y los árboles (41 spp.). El trabajo incluye información acerca de la disponibilidad espacial y temporal de las especies útiles, así como también de la importancia relativa de las de mayor interés para la población local, como son las combustibles, las medicinales, las alimenticias y las forrajeras.

Palabras clave: etnobotánica, México, Popolocas, Tehuacán.

\begin{abstract}
In this paper, we document the role and potential of plant resources in the Popoloca community of Zapotitlán Salinas, Puebla, located in the most arid zone of the TehuacánCuicatlán Valley. In total, 298 species belonging to 82 families were found. Ethnobotanical information is included for 288 species belonging to 219 genera and 79 botanical families, whose uses are placed in 19 categories. A total of 164 species are wild plants growing in different natural plant communities and in some of the transformed areas, while 124 can be found exclusively in the homegardens. The families with the highest number of useful
\end{abstract}


plants were Poaceae (35 spp.), Cactaceae (24 spp.), and Asteraceae (23 spp.). Most of the useful plants are herbs (162 spp.), followed by shrubs (86 spp.) and trees (41 spp.). The paper includes information about spatial and seasonal availability of the useful species, as well as data concerning relative importance of the most important ones, such as those used as medicine, food, firewood and fodder.

Keywords: ethnobotany, Mexico, Popolocas, Tehuacán.

\section{INTRODUCCIÓN}

Los estudios etnobotánicos en comarcas secas son relativamente escasos, sobre todo cuando se comparan con los realizados en zonas cálido-húmedas. Una de las regiones áridas de México que merecen especial atención desde esta perspectiva es el Valle de Tehuacán-Cuicatlán. Así lo demuestran, por una parte, su riqueza biológica, representada por altos niveles de diversidad y endemismo, tanto en grupos vegetales como animales (Dávila et al., 2002) y, por otra, la diversidad cultural de sus habitantes (Casas y Valiente-Banuet, 1995; Casas et al., 1997a, 1997b), así como su importancia en el contexto de la domesticación de plantas y origen de la agricultura en el Nuevo Mundo (MacNeish, 1967, 1992).

Aun cuando lo antes mencionado hace evidente la importancia etnobotánica del Valle de Tehuacán, en contraste con los avances alcanzados en el conocimiento florístico de la región (Dávila et al., 1993, 2002), son pocas las investigaciones que registran información sobre los usos de las plantas (Arias-Toledo, 2000; Casas y Valiente-Banuet, 1995; Casas et al., 1997a, 1997b, 2001; Miranda, 1948; Pardo-Núñez, 2001; Ramírez, 1996; Smith, 1965, 1967). No obstante lo anterior, los datos existentes sugieren que la magnitud de recursos vegetales aprovechados por los grupos humanos que habitan el Valle de Tehuacán-Cuicatlán es muy elevada. Así lo demuestra el reciente trabajo de Casas et al. (2001), quienes en una primera aproximación lograron identificar 808 especies vegetales útiles dentro de los límites geográficos de esta región.

El objetivo principal de la presente investigación es contribuir a incrementar el conocimiento de la flora útil de esta importante región de México, mediante la documentación del papel y del potencial de los recursos vegetales de Zapotitlán Salinas, Puebla, una comunidad popoloca ubicada en la porción más árida del Valle de Tehuacán-Cuicatlán. Los antecedentes de estudios etnobotánicos particulares para esta zona están representados por trabajos que no fueron hechos de manera exclusiva en el pueblo de Zapotitlán, sino que abarcaron a varios poblados del municipio, 
tal es el caso del de Ramírez (1996), quien registra 69 especies medicinales, del de Arias-Toledo (2000), quien documenta 58 especies útiles de la zona y, del de PardoNúñez (2001), que describe el uso de 72 plantas comestibles para todo el Valle de Tehuacán-Cuicatlán, de las cuales 47 se citan para el municipio de Zapotitlán.

Como objetivos particulares se contemplaron:

1. Documentar los criterios de percepción y clasificación de los componentes del medio natural entre los pobladores de la zona.

2. Hacer un inventario de la flora útil de Zapotitlán Salinas, Puebla, el cual incluyó los nombres comunes asignados a las especies registradas, los usos a los que se destinan, las partes de las plantas que son utilizadas, la(s) forma(s) de preparación y el tipo de manejo a que son sometidas.

3. Evaluar la disponibilidad espacial y temporal de los recursos vegetales de la zona y estimar la importancia relativa de algunos de ellos que son de mayor interés para la población local (combustibles, medicinales, alimenticios y forrajeras).

4. Evaluar la importancia de las unidades ambientales más importantes en la zona, en términos de su productividad de recursos vegetales.

\section{ZONA DE ESTUDIO}

La comunidad de Zapotitlán Salinas es la cabecera del municipio del mismo nombre (Fig. 1). Se encuentra enclavada en un valle ubicado en la porción occidental del Valle de Tehuacán-Cuicatlán, ocupando una superficie de $485 \mathrm{~km}^{2}$. Se caracteriza por la temperatura media anual de alrededor de $21^{\circ} \mathrm{C}$, con una canícula bien definida a la mitad del período de lluvias y la precipitación media anual de 400 a $450 \mathrm{~mm}$. Desde el punto de vista edáfico, en la mayor parte del área los suelos son someros, pedregosos y muestran diferentes niveles de alcalinidad y salinidad, producto de la influencia de los diferentes substratos geológicos presentes en el sitio. Las principales unidades de suelos citadas para la región son: litosoles, cambisoles cálcicos y xerosoles cálcicos derivados de evaporitas del Cretácico Inferior y Medio, complementados con regosoles y fluvisoles calcáricos formados por materiales transportados, derivados de sedimentos aluviales (López-Galindo et. al., 2003; Oliveros-Galindo, 2000). Las comunidades vegetales localizadas en la región de Zapotitlán son el mezquital o selva baja perennifolia con espinas laterales (con prevalencia de Prosopis laevigata); el matorral espinoso (con Mimosa luisana, Acacia farnesiana, Cordia curassavica y Fouquieria formosa); la tetechera (asociación con abundancia de Neobuxbaumia tetetzo), el cardonal (en el que destaca Cephalocereus 


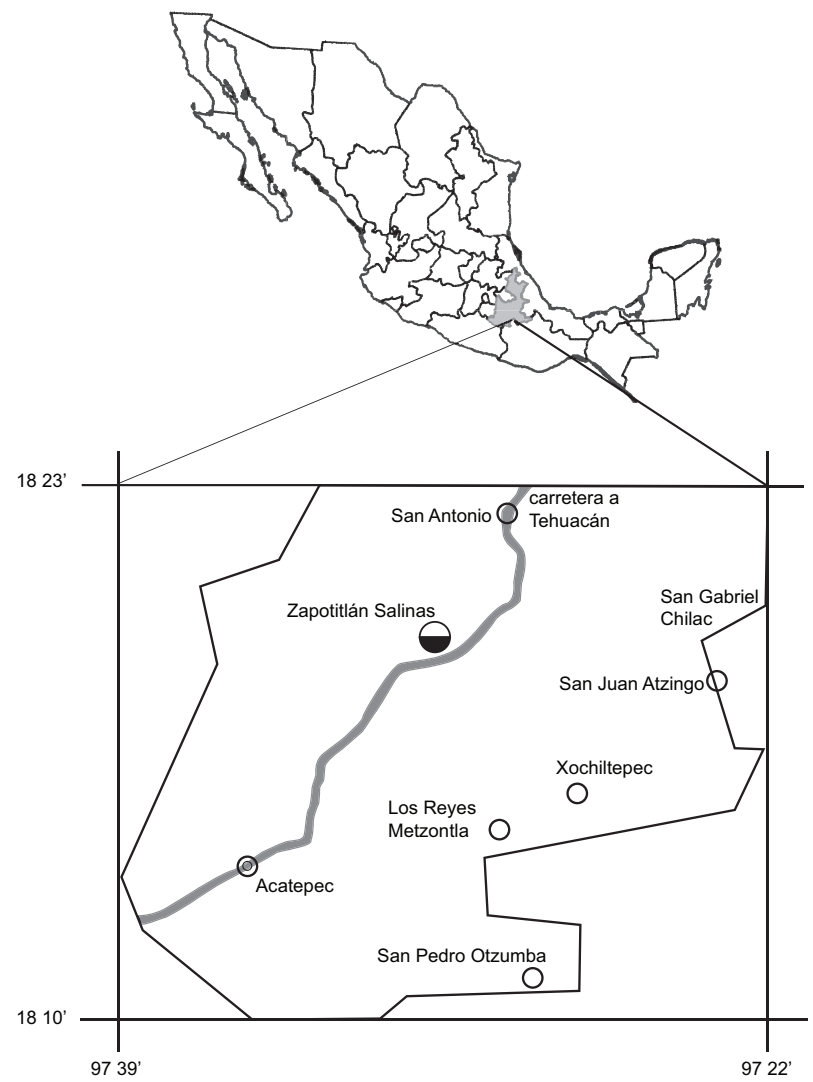

Fig. 1. Municipio de Zapotitlán de Salinas, Puebla.

columna-trajani); el izotal (matorral con Yucca periculosa); el chaparral o mexical (con Brahea nitida y Dasylirion serratifolium); la tetechera-candelillar (con Neobuxbaumia tetetzo y Euphorbia antisyphilitica), la selva baja caducifolia (con Myrtillocactus geometrizans, Bursera schlechtendalii y Bursera aptera) (Oliveros-Galindo, 2000; Osorio-Beristain et al., 1996; Valiente-Banuet et al., 2000).

La población de Zapotitlán es de origen popoloca, aunque en la actualidad muy pocos de sus más de 8000 habitantes (Anónimo, 1996) conocen su lengua original o si la conocen no gustan mucho de emplearla para comunicarse, lo cual ocurre también con muchos otros aspectos de su cultura (Vázquez, 1982). Los pobladores de Zapotitlán tienen ocupaciones diversas, las cuales incluyen la agricultura, la recolección de leña y de algunas plantas medicinales, la artesanía de ónix, la extrac- 
ción de sal, la albañilería y, en los últimos años, la prestación de mano de obra en la industria textil, la cual cuenta con cinco fábricas en el pueblo. Como en muchas otras partes de México, en los últimos años se ha incrementado la emigración de los hombres de Zapotitlán hacia los Estados Unidos.

\section{MÉTODOS}

El trabajo de campo se llevó a cabo entre febrero de 1998 y marzo de 2001 con salidas de 7 a 15 días por mes. Las actividades desarrolladas contemplaron varios aspectos metodológicos generales propuestos por Martin (1995). Así, para el inventario etnobotánico se hicieron colectas y entrevistas abiertas en las diferentes unidades ambientales naturales (tanto tipos de vegetación, como las localmente reconocidas) y transformadas (huertos, campos de cultivo, potreros) y se tuvo participación en diversas actividades relacionadas con el empleo y práctica de manejo de las plantas útiles de la zona. La mayor parte de las colectas se hicieron en compañía de alguno o varios informantes. Para cada una de las especies registradas como utilizables, se obtuvieron datos acerca del nombre común, forma de vida, usos, época de producción de las partes aprovechadas y tipo de manejo. Un total de 150 personas proporcionó información etnobotánica. Simultáneamente a este trabajo general, se realizó un estudio de huertos de Zapotitlán (Lee et al., en prep.), el cual será publicado posteriormente, pero cuyos datos más relevantes se consignan aquí. Durante el trabajo de campo se recolectaron 351 ejemplares de las especies registradas con usos, de los cuales un juego completo se depositó en el herbario IZTA y sus duplicados se encuentran en proceso de distribución a los herbarios ENCB y MEXU.

La determinación de la abundancia relativa y disponibilidad espacial de las plantas útiles sólo se hizo para las especies arbóreas y arbustivas (en este ejercicio también se incluyen los taxa que no tienen usos pero que forman parte de la vegetación). La información se obtuvo mediante muestreos en sitios representativos de los diferentes tipos de vegetación, de los cuales la población hace uso de los recursos vegetales. Para ello se emplearon transectos de $50 \mathrm{~m}$ de largo y $10 \mathrm{~m}$ de ancho $(5 \mathrm{~m}$ a cada lado de la línea), los cuales se subdividieron cada $10 \mathrm{~m}$, con lo que se obtuvieron 5 cuadros de $100 \mathrm{~m}^{2}$. En cada una de estas superficies se registraron todas las especies de árboles y arbustos y definieron los datos de abundancia (número de individuos) y frecuencia. La información etnobotánica referente a las épocas de producción de las partes utilizadas de las plantas comestibles se empleó para analizar la disponibilidad temporal de esas especies. 
La estimación de la importancia relativa de los productos utilizados se hizo mediante la aplicación del método etnográfico estructurado denominado enlistado libre (Alexiades, 1996; Bernard, 1994; Martin, 1995). Esta encuesta se aplicó a 25 informantes que estuvieron dispuestos a participar en la entrevista, de los cuales seis (24\%) se encuentran en el intervalo de edades de 8-20 años, otros seis (24\%) entre los 21-40 años y 13 (52\%) entre 41-89 años. La ocupación de los informantes fue: 14 amas de casa (45\%), 6 tenderos (3\%), 5 estudiantes (16\%), 8 campesinos (26\%) y 3 obreros (10\%); cabe mencionar que algunos de los entrevistados poseen más de una ocupación o actividad económica. En cuanto al sexo, 14 fueron mujeres (56\%) y 11 hombres (44\%) y, por último, respecto al lugar de nacimiento, 23 de los informantes (92\%) son originarios de Zapotitlán y sólo dos (8\%) son de otros lugares como Veracruz, o zonas cercanas a Zapotitlán. La entrevista consistió en solicitarles que elaboraran una lista de las 10-15 plantas que consideraran como de mayor importancia en los siguientes rubros: alimento, forraje, medicina y leña. En el caso de las medicinales, la encuesta se enfocó a determinar las enfermedades consideradas como las más importantes de la zona y el número de especies empleadas para su curación.

\section{RESULTADOS Y DISCUSIÓN}

Clasificación tradicional del medio natural

Los pobladores de Zapotitlán reconocen diferentes unidades ambientales en su entorno natural, a las cuales catalogan por características tales como la pendiente, el suelo, la pedregosidad, el uso al que se destinan y la altitud en la que se encuentran ubicadas. La figura 2 esquematiza dichas unidades y sus características se describen a continuación.

Cerros. Están en las partes más altas del área de estudio (1800-2400 m s.n.m.), sus suelos son de color negro, y se dice que, de no presentar tantas piedras, serían los mejores para sembrar. Las comunidades vegetales en estos sitios corresponden a chaparrales, selvas bajas e izotales, de donde se extraen 31 plantas útiles, entre las que se encuentran las medicinales (Heterotheca inuloides, Margaranthus solanaceus), las combustibles (Dasylirion serratifolium, Quercus sp.), las alimenticias (Porophyllum tagetoides) y las de uso artesanal (Brahea dulcis). En las porciones planas de los cerros se localizan sitios con influencia humana que incluyen cultivos de maguey (Agave atrovirens) y corrales de ganado caprino de libre pastoreo; los 
Paredes-Flores et al.: Estudio etnobotánico de Zapotitlán Salinas, Puebla

\begin{tabular}{|c|c|c|c|}
\hline \multirow[b]{2}{*}{$\begin{array}{c}2400 \\
\mathrm{~m} \text { s.n.m. } \\
1950- \\
\\
1500 \\
\end{array}$} & & & MONTE \\
\hline & 화 & - (18) & 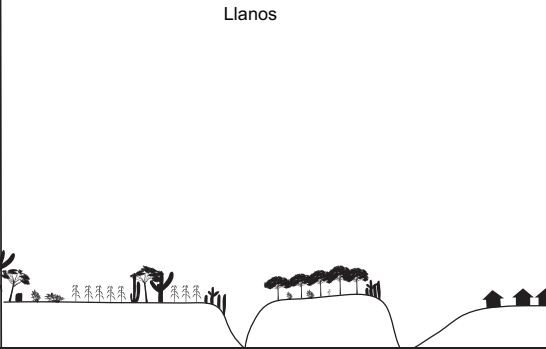 \\
\hline & $\begin{array}{l}\text { Cultivo de Agave } \\
\text { para la producción } \\
\text { de pulque. } \\
\text { Extracción de } \\
\text { plantas medicina- } \\
\text { les, comestibles. } \\
\text { Extracción de } \\
\text { palmas para } \\
\text { fabricación de } \\
\text { artesanías } \\
\text { (petates y } \\
\text { sopladores). } \\
\text { Pastoreo de } \\
\text { chivos. } \\
\text { Tipos de } \\
\text { vegetación: } \\
\text { chaparral, selva } \\
\text { baja caducifolia e } \\
\text { izotal. } \\
\text { Núm. de especies } \\
\text { útiles: } 31 .\end{array}$ & $\begin{array}{l}\text { Extracción de leña. } \\
\text { Extracción de plantas } \\
\text { medicianles y frutos } \\
\text { comestibles tales } \\
\text { como garambullos } \\
\text { y xoconostles. } \\
\text { Extracción de ónix. } \\
\text { Extracción de sal. } \\
\text { Pastoreo de chivos, } \\
\text { vacas, caballos y } \\
\text { burros. } \\
\text { Extracción de agaves } \\
\text { para preparación de } \\
\text { mezcal, y de sus } \\
\text { inflorescencias para } \\
\text { su venta. } \\
\text { Tipos de vegetación: } \\
\text { matorral espinoso, } \\
\text { mezquital, tetechera, } \\
\text { cardonal, tetechera- } \\
\text { candelillar. } \\
\text { Núm. de especies } \\
\text { útiles: } 185 .\end{array}$ & $\begin{array}{l}\text { Extracción de leña y de algunas } \\
\text { plantas comestibles de temporada, tales } \\
\text { como las verdolagas y quelites. } \\
\text { Extracción de frutos comestibles. } \\
\text { Cultivos de maíz, frijol y calabazas. } \\
\text { Huertas de limón, pitahaya y guayabas. } \\
\text { Huertos. } \\
\text { Tipos de vegetación: mezquital, matorral } \\
\text { espinoso. } \\
\text { Núm. de especies útiles: } 205 .\end{array}$ \\
\hline
\end{tabular}

Fig. 2. Representación esquemática y rasgos relevantes de las zonas del medio natural reconocidas por los habitantes de Zapotitlán Salinas, Puebla. 
sitios en donde se ubican estos últimos se van rotando cada determinado tiempo, con el objeto de fertilizar el suelo para el propio cultivo del maguey.

Monte. Bajo esta categoría se reconocen dos unidades: lomas y llanos.

Lomas. Estas geoformas se localizan a menor altitud que los cerros (1800$1650 \mathrm{~m}$ s.n.m.). Son muy variables en cuanto al tipo de suelo y a la inclinación del terreno, que puede ser muy pronunciada. Presentan abundancia de piedras, lo cual impide que sean usadas en su totalidad para cultivos, siendo las excepciones el maíz y el frijol. Las comunidades vegetales en estos sitios incluyen el matorral espinoso, el mezquital, la tetechera, el cardonal y la tetechera-candelillar, de donde se aprovechan 185 especies de plantas útiles, entre las que se incluyen medicinales como el ítamo real (Turnera diffusa) y el orégano (Lippia graveolens), alimenticias como la chupandía (Cyrtocarpa procera) y cinco negritos (Lantana camara) y para leña como el cumito (Mimosa luisana) y el garambullo (Myrtillocactus geometrizans). Las zonas con influencia humana incluyen potreros destinados a mantener la crianza de ganado vacuno, equino y asnar, así como también sitios planos destinados al cultivo de maíz y frijol principalmente.

Llanos. Estos ambientes se presentan aún a menor altitud $(1700$ a $1450 \mathrm{~m}$ s.n.m.) que las lomas, sus pendientes son más suaves y los suelos son de color gris. Se ubican en los bordes del cauce del Río Salado y se subdividen en terrones (terrazas aluviales) y hoyas, que son las formaciones que resultan de la erosión de los terrones. En los llanos dominan las superficies alteradas, en donde una buena parte se destina a la milpa, en la cual se cultivan principalmente maíz, frijol y pitahaya. En esta áreas se localizan el mezquital y matorral espinoso, de donde se extraen 205 especies útiles, entre las que están plantas comestibles como el garambullo (Myrtillocactus geometrizans) y medicinales como la malinche (Pachycereus marginatus) y el chimalacate (Viguiera dentata).

Barrancas. Estas son las partes más bajas de la zona y corresponden propiamente a los cauces de los riachuelos estacionales. En tales sitios pueden encontrarse algunas de las plantas utilizadas para el forrajeo de los chivos y medicinales como el popote (Gymnosperma glutinosum) y el cazahuate (Ipomoea pauciflora).

Inventario etnobotánico

Como se muestra en el Apéndice 1, se registraron usos de un total de 288 especies pertenecientes a 219 géneros y 79 familias botánicas, de las cuales 164 prosperan en las distintas comunidades vegetales naturales de la zona y en algunas de las áreas transformadas, mientras que las restantes (124) se encuentran de manera 
exclusiva en los huertos. Las familias con mayor número de especies fueron Poaceae (35 spp., 12.11\%), Cactaceae (24 spp, 8.3\%) y Asteraceae (23 spp., 7.96\%). En cuanto a las formas de vida, la mayor parte (162 spp.) corresponde a las herbáceas, seguida de los arbustos (86 spp.) y los árboles (41 spp.).

Los usos a los que se destinan las especies se pueden ubicar en 19 categorías, entre las que destacan las medicinales (98 spp., 22.48\%), las ornamentales (94 spp., $21.56 \%$ ), las forrajeras (90 spp., 20.64\%), las comestibles (82 spp., 18.81\%) y las empleadas como leña o combustible (25 spp., 5.73\%). Aunque es difícil hacer una comparación directa con los estudios previos realizados en la zona de estudio pues, como ya se ha señalado, esas investigaciones abarcaron a otros poblados del municipio de Zapotitlán, vale la pena señalar que dentro de las 164 especies silvestres útiles enlistadas en este trabajo, se incluyen 46 de las 58 mencionadas en la tesis de Arias-Toledo (2000); asimismo, en el caso de las 98 plantas medicinales y de las 82 comestibles registradas aquí para el poblado de Zapotitlán, se encuentran 55 de las 69 inventariadas por Ramírez (1996) y 37 de las consignadas en la contribución de Pardo-Núñez (2001).

Las partes más utilizadas son las hojas (127 spp.), seguidas de los frutos (75 spp.), las plantas completas (70 spp.) y las flores (47 spp.). Un total de 106 especies se emplean en forma múltiple e integral, es decir que se utilizan para más de un propósito y para muchas de ellas más de una de sus partes se destinan a uno o más usos (véase Apéndice). Ejemplos de ello son el coahuino (Schinus molle), también llamado jovino o pirul, el cual es aprovechado para leña, sombra y como medicina, además de que se destina al forraje y la construcción. Otros ejemplos son el garambullo (Myrtillocactus geometrizans), la pitaya (Stenocereus pruinosus) y el xoconostle (Stenocereus stellatus), tres cactáceas columnares que se utilizan como alimento, forraje, leña y cerca viva. De acuerdo con lo consignado por otros autores, el uso múltiple e integral que se hace de las especies vegetales en Zapotitlán, parece ser una práctica común, no sólo de manera particular en el Valle de Tehuacán, sino también en general en los pueblos de Mesoamérica (Alcorn, 1984; Casas et. al., 1997a, 1997b, 2001; Colunga-García Marín, 1984; Williams, 1985, entre otros).

En cuanto a la nomenclatura tradicional registrada, los datos obtenidos indican que las 288 especies se reconocen con nombres en español, mientras que sólo 20 tienen asignado algún nombre en popoloca u otra lengua. Dentro de estas últimas destacan algunas plantas medicinales, que poseen nombres en ambos idiomas como la espinosilla o katsjo morado (Loeselia coerulea), el gigante o kanda xanttingani (Nicotiana glauca) y la lágrima o kandachansa (Sedum dendroideum). 
Importancia relativa de las especies útiles

Plantas alimenticias. De entre las 82 especies comestibles registradas, las más apreciadas son el maíz (Zea mays), el garambullo (Myrtillocactus geometrizans), el frijol (Phaseolus vulgaris) y la pitahaya (Hylocereus undatus) (Cuadro 1). La importancia del maíz y el frijol no es sorprendente, pues se trata de los principales elementos en la dieta de los mexicanos. En contraste, el garambullo y la pitahaya son alimentos de importancia local, de los cuales, además, se llegan a obtener beneficios económicos por la venta de sus productos. Otras especies alimenticias de significancia son las verdolagas (Portulaca oleracea), los palmitos (Yucca periculosa) y la chondata (Acacia acatlensis).

Cuadro 1. Importancia relativa de las plantas usadas como comestibles en Zapotitlán Salinas, Puebla (muestra de 25 entrevistados).

\begin{tabular}{|l|l|c|c|}
\hline Especies comestibles & Nombres comunes & $\begin{array}{c}\text { Núm. de } \\
\text { menciones }\end{array}$ & $\begin{array}{c}\text { Porcentaje } \\
(\%)\end{array}$ \\
\hline Zea mays & Maíz & 14 & 7.1 \\
\hline Myrtillocactus geometrizans & Garambullo & 14 & 7.1 \\
\hline Phaseolus vulgaris & Frijol & 14 & 7.1 \\
\hline Hylocereus undatus & Pitahaya & 10 & 5.1 \\
\hline Neobuxbaumia tetetzo & Tetecho & 8 & 4.1 \\
\hline Portulaca oleracea & Verdolaga & 7 & 3.6 \\
\hline Yucca periculosa & Palmitos, Izote & 7 & 3.6 \\
\hline Acacia acatlensis & Chondata & 7 & 3.6 \\
\hline $\begin{array}{l}\text { Cucurbita moschata } \\
\text { Cucurbita pepo }\end{array}$ & $\begin{array}{l}\text { Calabaza tamalayota, } \\
\text { Calabaza de castilla }\end{array}$ & 7 & 3.6 \\
\hline Agave peacockii & Cacaya (Maguey del ixtle) & 7 & 3.6 \\
\hline $\begin{array}{l}\text { Opuntia ficus-indica } \\
\text { Opuntia streptacantha }\end{array}$ & $\begin{array}{l}\text { Nopal de huerta } \\
\text { Nopal de tuna roja }\end{array}$ & 6 & 3.0 \\
\hline Amaranthus hybridus & Quelite & 6 & 3.0 \\
\hline Stenocereus pruinosus & Pitaya & 6 & 3.0 \\
\hline Leucaena esculenta & Huaje rojo & 6 & 3.0 \\
\hline Leucaena leucocephala & Huaje blanco & 6 & 3.0 \\
\hline Otras & & 72 & 36.5 \\
\hline
\end{tabular}


Plantas forrajeras. En esta categoría de uso se incluyen todos los vegetales, cultivados o silvestres, que son consumidos por los animales y especialmente por el ganado caprino. Las plantas comúnmente conocidas con el nombre genérico de pastos (varias especies de la familia Poaceae), además del rastrojo derivado de la cosecha del maíz, resultaron ser las de mayor importancia en este rubro. Otras especies también significativas en la alimentación del ganado caprino son el mezquite (Prosopis laevigata), la alfalfa (Medicago sativa) y la lechuguilla (Hechtia podantha) (Cuadro 2). De todas ellas se aprovechan las hojas y otras partes vegetativas, mientras que del mezquite se utilizan también los frutos, los cuales incluso se colectan y almacenan para la comida de los animales domésticos en épocas de sequía.

Cuadro 2. Importancia relativa de las plantas usadas como forrajeras en Zapotitlán Salinas, Puebla (muestra de 25 entrevistados).

\begin{tabular}{|l|l|c|c|}
\hline Especies forrajeras & Nombres populares & $\begin{array}{c}\text { Número de } \\
\text { menciones }\end{array}$ & $\begin{array}{c}\text { Porcentaje } \\
(\%)\end{array}$ \\
\hline Zea mays & $\begin{array}{l}\text { Maíz (sólo los residuos de } \\
\text { la cosecha) }\end{array}$ & 16 & 15 \\
\hline Medicago sativa & Alfalfa & 11 & 10 \\
\hline Amaranthus hybridus & Quelite & 9 & 8 \\
\hline Poaceae (varias especies) & Pasto & 8 & 7 \\
\hline Prosopis laevigata & Mezquite & 8 & 7 \\
\hline Hechtia podantha & Lechuguilla & 8 & 7 \\
\hline Parkinsonia preacox & Manteco, Palo verde & 5 & 5 \\
\hline Tithonia tubiformis & Acahuale & 3 & 3 \\
\hline Echinocactus platyacanthus & Biznaga & 3 & 3 \\
\hline $\begin{array}{l}\text { Opuntia decumbens } \\
\text { Opuntia ficus-indica }\end{array}$ & $\begin{array}{l}\text { Nopal de coyote } \\
\text { Nopal de huerta }\end{array}$ & 3 & 3 \\
\hline Acacia constricta & Guajillo & 3 & 3 \\
\hline Lippia graveolens & Orégano & 2 & 2 \\
\hline Sonchus oleraceaus & Achicoria & 2 & 2 \\
\hline Viguiera dentata & Chimalacate & 23 \\
\hline Otras & & 2 & 2 \\
\hline
\end{tabular}


Plantas combustibles. En el caso de las especies empleadas como combustible, las más importantes resultaron ser el mezquite, el cumito (Mimosa luisana) y el manteco (Parkinsonia praecox), además de algunas cactáceas columnares como el tetecho (Neobuxbaumia tetetzo), el baboso (Pachycereus hollianus) y el garambullo (Cuadro 3). De acuerdo con la información recabada, el valor atribuido a todas ellas como leña se debe a su duración, a la cantidad de calor que producen y a los beneficios económicos que se derivan de su venta o intercambio, tanto local como en los mercados regionales. En contraste, la madera de varias especies como el pirul (Schinus molle), no obstante que también se utiliza como leña, se considera de mala calidad, ya que su combustión produce una gran cantidad de humo. Vale la pena señalar, sin embargo, que algunas personas tienen cierto aprecio por la leña de esta última especie para preparar barbacoa, pues aseguran que le da un mejor sabor a la carne. La leña representa para la comunidad una fuente importante de energía doméstica y un ahorro económico sustancial, por lo que podría considerarse como un recurso fundamental e imprescindible dentro de la misma. Lo anterior pudo corroborarse con las encuestas practicadas, ya que $36 \%$ de los entrevistados indicaron que usaban exclusivamente leña como combustible, mientras que $44 \%$ usan gas y leña (aunque prefieren la leña para algunas prácticas como la cocción del maíz para la masa) y sólo $20 \%$ usan únicamente gas.

Cuadro 3. Importancia relativa de las plantas usadas como combustibles en Zapotitlán Salinas, Puebla (muestra de 25 entrevistados).

\begin{tabular}{|l|l|c|c|}
\hline Especies combustibles & Nombres comunes & $\begin{array}{c}\text { Núm. de } \\
\text { menciones }\end{array}$ & $\begin{array}{c}\text { Porcentaje } \\
(\%)\end{array}$ \\
\hline Prosopis laevigata & Mezquite & 22 & 14 \\
\hline Mimosa luisana & Cumito & 16 & 10 \\
\hline Parkinsonia praecox & Manteco & 15 & 10 \\
\hline Schinus molle & Pirul & 14 & 9 \\
\hline Neobuxbaumia tetezo & Tetecho & 13 & 8 \\
\hline Acacia constricta & Guajillo & 11 & 7 \\
\hline Myrtillocactus geometrizans & Garambullo & 11 & 7 \\
\hline Pachycereus hollianus & Baboso & 5 & 3 \\
\hline Lippia graveolens & Orégano & 5 & 3 \\
\hline Celtis pallida & Biscolote, Hoja de Parra & 4 & 3 \\
\hline Pachycereus marginatus & Órgano, Malinche & 4 & 3 \\
\hline Lysiloma divaricata & Palo blanco & 3 & 2 \\
\hline Ceiba parvifolia & Pochote & 3 & 2 \\
\hline Otras & & 31 & 19 \\
\hline
\end{tabular}


Enfermedades y plantas medicinales. La encuesta referente a las enfermedades de mayor incidencia entre la población de Zapotitlán reveló que los padecimientos considerados como los más importantes son el dolor de estómago, la gripa, la tos y la diarrea (Cuadro 4). Es interesante mencionar que algunos de estos datos coinciden con los proporcionados por el médico de la Unidad Médico Familiar Rural del Instituto Mexicano del Seguro Social (Cuadro 5) y se refleja en el gran número de plantas medicinales usadas para la curación de estos padecimientos y/o síntomas (Cuadro 6). En el caso de las dolencias gastrointestinales destacan el orégano (Lippia graveolens) y el ítamo real (Turnera diffusa), mientras que para las otras enfermedades son muy apreciadas la lengua de conejo (Sedum allantoides), el popote (Gymnosperma glutinosum) y el barredor (Cordia curassavica). Por otra parte, se encontró que existen síntomas que la población reconoce como enfermedades diferentes a las antes mencionadas y para cuya curación tienen plantas específicas. Ejemplo de ello es el caso del dolor de cabeza, para el cual se emplean las hojas frescas del gigante (Nicotiana glauca). Adicionalmente, hay una serie de padecimientos que pudieran considerarse de filiación cultural, los cuales no entran dentro del cuadro básico de enfermedades que trata la medicina oficial y entre los que se puede mencionar el "aire", para cuyo tratamiento se usa la ruda (Ruta chalepensis).

Cuadro 4. Importancia relativa de las enfermedades que se presentan en Zapotitlán, de acuerdo con la información obtenida en las entrevistas (muestra de 25 entrevistados).

\begin{tabular}{|l|c|c|}
\hline Enfermedades & Menciones & $(\%)$ \\
\hline Otras & 26 & 23 \\
\hline Dolor de estómago & 14 & 13 \\
\hline Gripa & 11 & 10 \\
\hline Tos & 10 & 9 \\
\hline Diarrea, disentería & 9 & 8 \\
\hline Diabetes & 7 & 6 \\
\hline Calentura & 6 & 6 \\
\hline Paperas-anginas & 5 & 5 \\
\hline Aire & 5 & 5 \\
\hline Dientes & 4 & 4 \\
\hline Quemada del sol & 4 & 4 \\
\hline Golpes & 4 & 4 \\
\hline Presión & 3 & 3 \\
\hline
\end{tabular}


Cuadro 5. Enfermedades de mayor incidencia en Zapotitlán, de acuerdo con la información proporcionada por la Unidad Médico Familiar Rural del Instituto Mexicano del Seguro Social.

\begin{tabular}{|l|}
\hline Faringitis \\
\hline Amigdalitis \\
\hline Gastroenteritis con deshidratación \\
\hline Diabetes \\
\hline Hipertensión arterial \\
\hline Parasitosis intestinal en menores de 5 años \\
\hline Picaduras por insectos \\
\hline Micosis en niños y micosis de uñas en la población general \\
\hline Varicela (abril-agosto) \\
\hline Intoxicación alimenticia \\
\hline
\end{tabular}

Cuadro 6. Número de plantas medicinales usadas en Zapotitlán para los diferentes aparatos, sistemas y padecimientos.

\begin{tabular}{|l|c|}
\hline Aparatos, sistemas y enfermedades & Especies \\
\hline Digestivo & 25 \\
\hline Músculo-esquelético & 18 \\
\hline Genito-urinario & 10 \\
\hline Piel & 8 \\
\hline Respiratorio & 7 \\
\hline Padecimientos de definición popular & 7 \\
\hline Calentura & 4 \\
\hline Circulatorio & 4 \\
\hline Metabólico & 4 \\
\hline Dolor de cabeza & 3 \\
\hline Nervioso & 2 \\
\hline Auditivo & 2 \\
\hline Oftálmico & 1 \\
\hline Total de especies & 95 \\
\hline
\end{tabular}

Manejo

De acuerdo con diversos autores (Alcorn, 1983; Bye, 1979; Casas et al., 1996; Colunga-García Marín, 1984; Davis y Bye, 1982; Williams, 1985, entre otros), es po- 
sible reconocer una amplia gama de interacciones hombre-planta, las cuales pueden ubicarse en dos categorías principales de manejo: in situ y ex situ. El primero implica actividades que se llevan a cabo en los mismos espacios ocupados por las poblaciones de plantas espontáneas y comprenden la simple recolección, la tolerancia, el fomento y la protección de las especies útiles. El segundo, por su parte, incluye interacciones que se llevan a cabo en hábitats creados y controlados por el hombre. Aunque estas formas de manejo se usan comúnmente con plantas domesticadas, también se aplican a las silvestres y arvenses. Las dos modalidades principales de manipulación ex situ son el trasplante y la siembra. Las especies vegetales registradas con usos en Zapotitlán se ubican en prácticamente todas las categorías antes mencionadas. Así, aunque la mayor parte de ellas (151 spp.) son plantas recolectadas de la vegetación natural de los alrededores de los poblados, existen también otras que son toleradas (17 spp.), fomentadas ( $9 \mathrm{spp}$.), protegidas ( $8 \mathrm{spp}$.), transplantadas (6 spp.) y cultivadas (130 spp.). Algunas especies, además, están sujetas a más de una forma de manejo y las siguientes son ejemplos de ello.

Echeveria gibbiflora y Sedum allantoides. Conocidas como "siempreviva orejona" y "lengua de conejo", respectivamente. Las dos se emplean como ornamentales y como medicinales. Aunque se trata de plantas silvestres, también es posible encontrarlas en algunos huertos. En ambos casos su manejo se inicia con el transplante de los individuos completos.

Agave marmorata. Conocida localmente como pitzomel, pichu y quiote, se utiliza como alimento, medicina, materia prima para la elaboración de bebida alcohólica y con fines industriales en la fabricación de nidos para las aves. Esta especie se fomenta "capando" el maguey, lo que implica que algún animal consuma la inflorescencia, en sus primeras etapas de desarrollo, con lo que se promueve la reproducción vegetativa y en consecuencia una mayor formación de nuevas plantas.

Capsicum annuum. Conocido como chili monte, es usado como comestible (especia). Además de recolectarse para su uso, recientemente se cultiva dentro de los huertos, mediante la siembra de semillas o el trasplante de individuos jóvenes o pobremente desarrollados que se encuentran creciendo como arvenses.

Disponibilidad temporal y espacial de las especies útiles

Como ya se ha señalado, el estudio sobre la disponibilidad temporal de las especies útiles se realizó con particular énfasis en las empleadas como alimento. Los datos recopilados revelaron que el mayor porcentaje de plantas y/o de sus partes comestibles, es aprovechable durante los meses de abril a agosto. 
En cuanto al estudio de disponibilidad espacial de la flora útil en su conjunto, se encontró que el mayor número de especies (191) crecen dentro de los huertos y entre ellas están las 124 que son exclusivas de estos sitios, además de otras 67 que pueden también localizarse en los distintos tipos de vegetación o en las diversas áreas transformadas de la zona. El segundo lugar lo ocupa el mezquital (62 spp.), mientras que el matorral espinoso y las áreas antropogénicas, como los campos de cultivo, incluyen 43 especies útiles cada una (Cuadro 7).

En lo que toca a la disponibilidad espacial, pero sólo de las especies arbóreas y arbustivas útiles, como ya se señaló se encontró que las unidades ambientales de mayor productividad resultaron ser las lomas y los llanos. Esto no es sorprendente, ya que dentro de ellas se localizan casi la totalidad de las comunidades vegetales de las cuales hace uso la población local (mezquitales, tetecheras, matorral espinoso, cardonal, tetechera-candelillar e izotal). Un total de 21 especies de árboles y arbustos se registraron con algún uso dentro de estas unidades ambientales, además de otras 45 inventariadas exclusivamente en los huertos.

Los resultados de los muestreos de los árboles y arbustos útiles presentes en los diferentes tipos de vegetación, por su parte, revelaron que las categorías de uso mejor representadas en todos esos sitios son: ornamentales, comestibles, medicinales, combustibles y forrajeras (Apéndice 2).

Por otra parte, los datos registrados indican que no parece existir una relación muy consistente entre alguno de los parámetros cuantificados en los muestreos de los distintos tipos de vegetación y el número de usos de las especies presentes en ellos. Así, en el mezquital la especie más abundante es Agave karwinskii, la cual únicamente se emplea como cerca viva. Le siguen Pachycereus hollianus, Prosopis laevigata, Myrtillocactus geometrizans, Castella erecta y Celtis pallida. Las tres primeras se aprovechan para varios propósitos, mientras que las últimas dos sólo se utilizan como medicinales. En el caso de la tetechera, las dos especies más abundantes (Neobuxbaumia tetetzo y Pachycereus hollianus) tienen cuatro usos, mientras que la siguiente más común es Castella erecta, la cual, como ya se señaló, únicamente se emplea como medicinal. Una situación similar se encontró para el cardonal, la tetechera-candelillar y el chaparral, en los cuales las plantas más comunes generalmente tienen registrados más de dos usos. En contraste, en el matorral espinoso y en el izotal las especies más abundantes no se inventariaron como útiles.

\section{COMENTARIOS FINALES}

No obstante la gran cantidad de cambios socio-culturales que han ocurrido en los últimos años en la comunidad popoloca que habita Zapotitlán Salinas (pérdida 
Paredes-Flores et al.: Estudio etnobotánico de Zapotitlán Salinas, Puebla

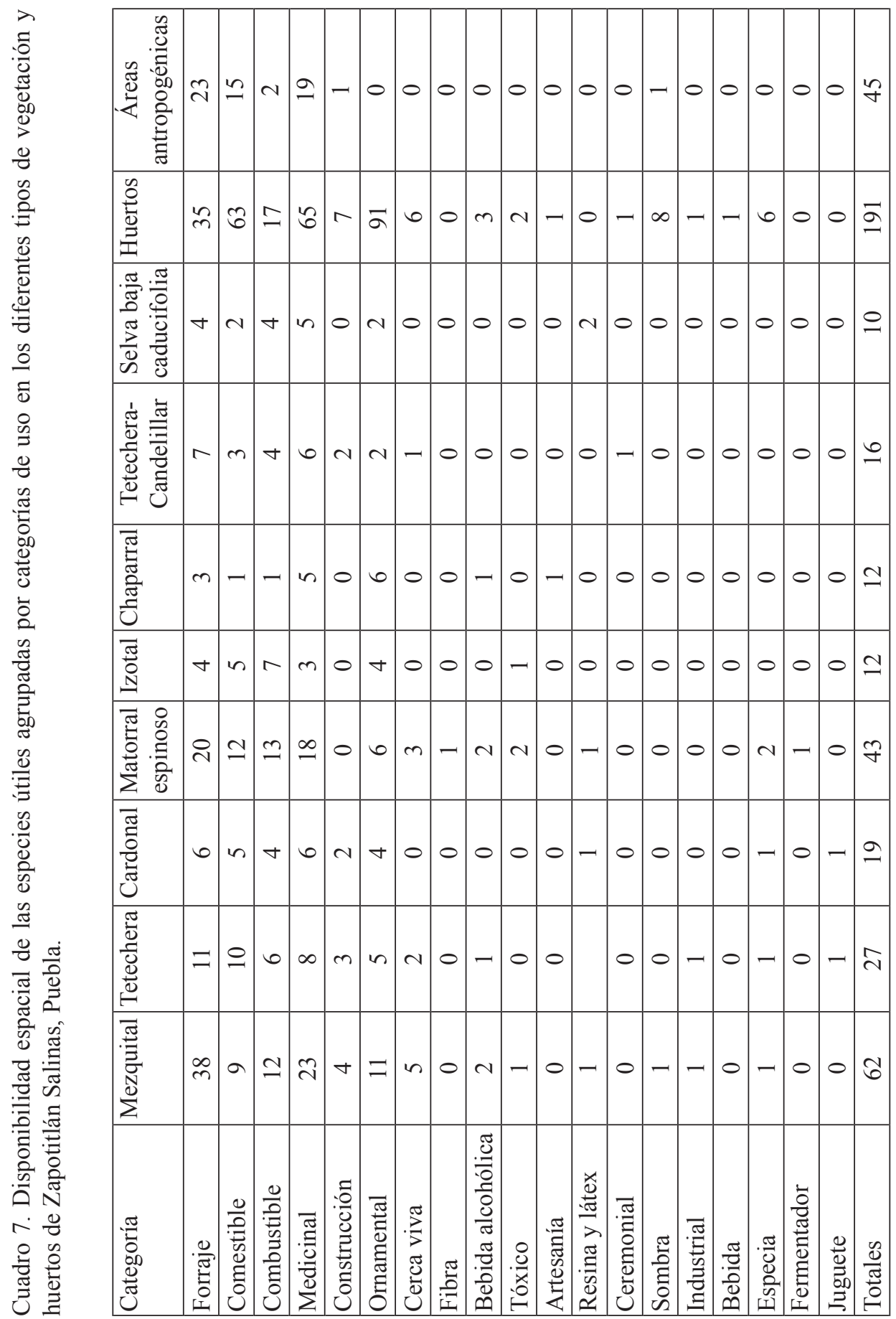


del lenguaje, migración, cambios de ocupación, etc.), los datos obtenidos en este trabajo revelan que sus habitantes siguen dependiendo del medio natural y poseen un gran entendimiento de las propiedades de la flora local de la zona. Lo anterior se refleja en el amplio conocimiento que tienen de su entorno natural y en el relativamente elevado número de especies silvestres utilizadas y la diversidad de usos a los que se destinan. Es de destacarse, por ejemplo, el hecho de que las categorías de empleo más importantes estén representadas en proporciones casi iguales. Este hecho, aunado a la gran cantidad de plantas que se utilizan en forma múltiple e integral, sugiere que ha existido y aún persiste una estrecha relación de los pobladores con la flora local.

Por otra parte, si bien es cierto que la mayor parte de las especies útiles son recolectadas, las interacciones de la gente de Zapotitlán con las plantas no se limitan a este tipo de apropiación de dichos recursos, sino que también involucran una variedad de prácticas de manejo, tanto in situ en las diferentes áreas naturales, como ex situ en donde los huertos representan una parte fundamental. En este contexto algunas especies destacan, pues están sometidas a más de una forma de tratamiento y un estudio detallado de todas ellas podría revelar procesos incipientes de domesticación.

\section{AGRADECIMIENTOS}

Para la realización de este trabajo, se contó con el apoyo del Consejo Nacional de Ciencia y Tecnología y de la Comisión Nacional Para el Conocimiento y Uso de la Biodiversidad, a través de los proyectos Los Recursos Vegetales del Valle de Tehuacán-Cuicatlán desde una Perspectiva Etnobotánica (Proyecto CONACyT G35450V) y Flora Útil de Dos Comunidades Indígenas del Valle de Tehuacán-Cuicatlán: Coxcatlán y Zapotitlán Salinas (Proyecto CONABIO T015). Los autores agradecen de manera especial a la población y a las autoridades de Zapotitlán por su apoyo y colaboración.

\section{LITERATURA CITADA}

Alcorn, J. B. 1983. Huastec non crop resource management: Implications for prehistoric rainforest management. Hum. Ecol. 9(4): 395-417.

Alcorn, J. B. 1984. Huastec Mayan ethnobotany. University of Texas Press. Austin. 982 pp.

Alexiades, M. N. 1996. Selected guidelines for ethnobotanical research: a field manual. The New York Botanical Garden. Nueva York. 306 pp. 
Anónimo. 1996. Cuaderno de información estadística del sector salud y seguridad social: Puebla. Instituto Nacional de Estadística, Geografía e Informática. Aguascalientes. $153 \mathrm{pp}$.

Arias-Toledo, A. 2000. Las plantas de Zapotitlán Salinas, Puebla: un folleto de divulgación y conservación. Tesis de licenciatura en biología. Facultad de Ciencias, Universidad Nacional Autónoma de México. México, D.F. 126 pp.

Bernard, H. R. 1994. Research methods in anthropology: qualitative and quantitative approaches. 2a. ed. Sage Publication. Thousand Oaks. Londres, Nueva Delhi. 585 pp.

Bye, R. A. 1979. Incipient domestication of mustards in northwest Mexico. Kiva 44: 237256.

Casas, A. y A. Valiente-Banuet. 1995. Etnias, recursos genéticos y desarrollo sustentable en zonas áridas de México. In: Anaya, M. y F. Díaz-Calero (eds.). IV curso sobre desertificación y desarrollo sustentable en América Latina y el Caribe. Red de Información Ambiental para América Latina y el Caribe (PNUMA) / Red de Cooperación Técnica en Zonas Áridas y Semiáridas de América Latina y el Caribe (FAO) / Colegio de Postgraduados (CP). Montecillos, México. 432 pp.

Casas, A., M. C. Vázquez, J. L. Viveros y J. Caballero. 1996. Plant management among the Nahua and the Mixtec of the Balsas river basin: an ethnobotanical approach to the study of plant domestication. Hum. Ecol. 24: 455-478.

Casas, A., B. Pickersgill, J. Caballero y A. Valiente-Banuet. 1997a. Ethnobotany and domestication in xoconochtli Stenocereus stellatus (Cactaceae) in the Tehuacan Valley and la Mixteca Baja, Mexico. Econ. Bot. 51(3): 279-292.

Casas, A., J. Caballero, C. Mapes y S. Zárate. 1997b. Manejo de la vegetación, domesticación de plantas y origen de la agricultura en Mesoamérica. Bol. Soc. Bot. Méx. 61: 31-47.

Casas, A., A. Valiente-Banuet, J. L. Viveros, J. Caballero, L. Cortés, P. Dávila, R. Lira e I. Rodríguez-Arévalo. 2001. Plant resources of the Tehuacan-Cuicatlan Valley, México. Econ. Bot. 55: 129-166.

Colunga-García Marín, P. 1984. Variación morfológica, manejo agrícola y grados de domesticación de Opuntia spp. en el Bajío Guanajuatense. Tesis de maestría en ciencias. Colegio de Postgraduados. Montecillos, México. 204 pp.

Dávila, P., J. L. Villaseñor, R. Medina, A. Ramírez, A. Salinas, J. Sánchez-Ken y P. Tenorio. 1993. Flora del Valle de Tehuacán-Cuicatlán. Listados florísticos de México X. Instituto de Biología, Universidad Nacional Autónoma de México, México, D.F. 195 pp.

Dávila, P., M. C. Arizmendi, A. Valiente-Banuet, J. L. Villaseñor, A. Casas y R. Lira. 2002. Biological diversity in the Tehuacan-Cuicatlan Valley, Mexico. Biodivers. Conserv. 11: 421-442.

Davis, T. y R. A. Bye. 1982. Ethnobotany and progressive domestication of Jaltomata (Solanaceae) in Mexico. Econ. Bot. 36: 225-241.

López-Galindo, F., D. Muñoz-Iniestra, M. Hernández-Moreno, A. Soler-Aburto, M. C. Castillo-López e I. Hernández-Arzate. 2003. Análisis integral de la toposecuencia y su influencia en la distribución de la vegetación y la degradación del suelo en la Subcuenca de Zapotitlán Salinas, Puebla. Bol. Soc. Geol. Mex. 56(1): 19-41. 
MacNeish, R. S. 1967. A summary of the subsistence. In: Byers, D. S. (ed.). The prehistory of the Tehuacan Valley. Vol. 1. Environment and subsistence. University of Texas Press. Austin. pp. 290-331.

MacNeish, R. S. 1992. The origins of agriculture and settled life. University of Oklahoma Press. Norman. 433 pp.

Martin, G. J. 1995. Ethnobotany. People and plants conservation manuals 1. World Wildlife Fund for Nature International (WWF), United Nations Educational, Scientific and Cultural Organisation (UNESCO), Royal Botanic Gradens, Kew. Chapman \& Hall. Londres. 268 pp.

Miranda, F. 1948. Datos sobre la vegetación en la Cuenca Alta del Papaloapan. An. Inst. Biol. Universidad Nacional Autónoma de México 19: 333-364.

Oliveros-Galindo, O. 2000. Descripción estructural de las comunidades vegetales en las terrazas aluviales del Río Salado, en el Valle de Zapotitlán de las Salinas, Puebla. Tesis de licenciatura en biología. Facultad de Estudios Superiores-Iztacala, Universidad Nacional Autónoma de México. Tlalnepantla, México. 87 pp.

Osorio-Beristain, O., A. Valiente-Banuet, P. Dávila y R. Medina. 1996. Tipos de vegetación y diversidad B en el Valle de Zapotitlán de las Salinas, Puebla, México. Bol. Soc. Bot. Méx. 59: 35-58.

Pardo-Núñez, J. 2001. Diagnóstico de las plantas silvestres, arvenses y ruderales que son empleadas como alimento por habitantes de cuatro localidades del Valle de TehuacánCuicatlán. Tesis de licenciatura en biología. Facultad de Estudios Superiores-Iztacala, Universidad Nacional Autónoma de México. Tlalnepantla, México. 109 pp.

Ramírez, H. A. 1996. Contribución al conocimiento de la flora medicinal de Zapotitlán de las Salinas, Puebla. Tesis de licenciatura en biología. Facultad de Ciencias, Universidad Nacional Autónoma de México. México, D.F. 94 pp.

Smith, C. E. 1965. Flora, Tehuacán Valley. Fieldiana Bot. 31: 101-143.

Smith, C. E. 1967. Plant remains. In: Byers, D. S. (ed.). The prehistory of the Tehuacán Valley. Vol. 1. Environment and subsistence. University of Texas Press. Austin. pp. 220-225.

Valiente-Banuet, A., A. Casas, A. Alcántara, P. Dávila, N. Flores-Hernández, J. L. Villaseñor y J. Ortega. 2001. La vegetación del Valle de Tehuacán-Cuicatlán. Bol. Soc. Bot. Méx. 67: 25-74.

Vázquez, M. H. 1982. Los popolocas. Instituto Nacional Indigenista. México, D.F. 10 pp.

Williams, D. E. 1985. Tres arvenses solanáceas comestibles y su proceso de domesticación en el estado de Tlaxcala, México. Tesis de maestría en ciencias. Colegio de Postgraduados. Chapingo, México. 173 pp.

Recibido en mayo de 2003. Aceptado en octubre de 2006. 
Paredes-Flores et al.: Estudio etnobotánico de Zapotitlán Salinas, Puebla $\|$ în

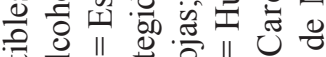

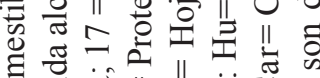

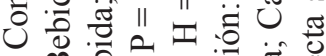
$\|$ की 人 a iิ

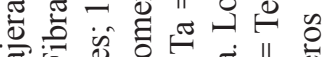
필

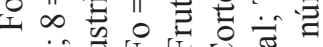

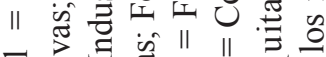
- .

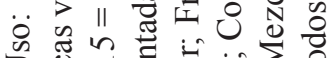

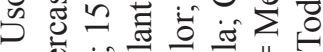

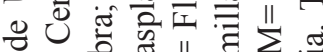
॥ है

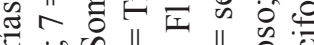

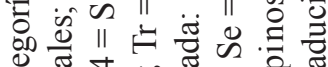

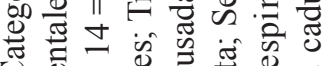

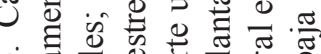

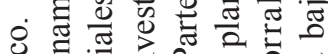
.

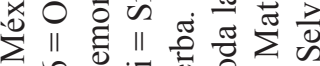

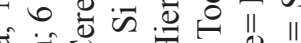
กี

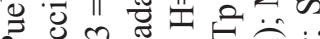

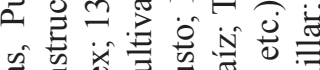

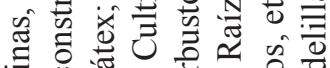
寻穴

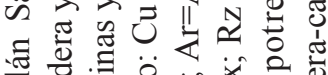

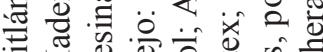

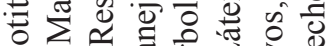

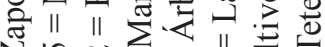

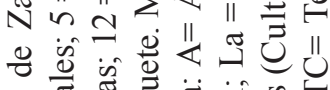

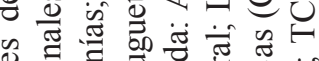

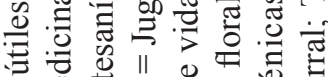

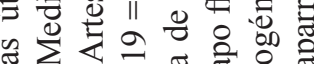

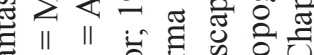

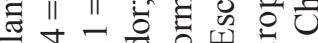

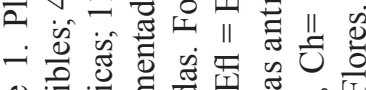
ठ :

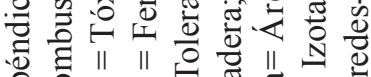

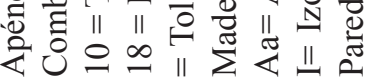

\begin{tabular}{|c|c|c|c|c|c|c|c|c|c|c|c|c|}
\hline 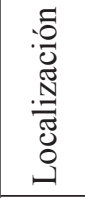 & & $\begin{array}{l}\sum^{0} \\
\sum^{0} \\
\sum^{E}\end{array}$ & 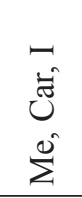 & & 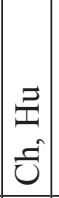 & $\left|\begin{array}{l}0 \\
\tilde{n} \\
\dot{\Sigma} \\
\tilde{\omega}\end{array}\right|$ & $\begin{array}{l}\vec{\Xi} \\
\dot{E} \\
\dot{\Sigma}\end{array}$ & $\sum^{0}$ & $\stackrel{0}{\Sigma}$ & 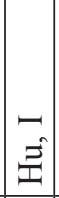 & $\stackrel{0}{\Sigma}$ & $\stackrel{\Xi}{\Xi}$ \\
\hline $\begin{array}{l}\frac{.0}{\stackrel{\Xi}{E}} \\
\sum\end{array}$ & & $\vec{U}$ & $\ddot{\sim}$ & & ت & $\dot{\sim n}$ & $\begin{array}{l}\dot{\sim} \\
0 \\
0 \\
\dot{I}\end{array}$ & $\bar{\sim}$ & $\begin{array}{l}\bar{n} \\
0 \\
0 \\
\tilde{I}\end{array}$ & $\begin{array}{l}\dot{F} \\
\dot{\omega}\end{array}$ & $\bar{\sim}$ & $\bar{\sim}$ \\
\hline 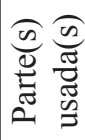 & & $\stackrel{\pi}{\stackrel{\pi}{a}}$ & e & & $\begin{array}{l}\vec{I} \\
\overrightarrow{\tilde{E}}\end{array}$ & $\begin{array}{l}\text { 厌 } \\
\text { تే }\end{array}$ & $\mid \begin{array}{l}\vec{I} \\
\vec{E} \\
\vec{E} \\
\tilde{I}\end{array}$ & $\begin{array}{l}\text { 死 } \\
\underline{I}\end{array}$ & $\begin{array}{l}\vec{I} \\
\underline{I}\end{array}$ & $\stackrel{2}{E}$ & 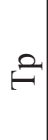 & II \\
\hline $\begin{array}{l}\mathscr{n} \\
\stackrel{0}{\infty} \\
\mathscr{D}\end{array}$ & & $\begin{array}{l}0 \\
\forall\end{array}$ & 6 & & $\begin{array}{l}\sigma \\
\sigma^{*}\end{array}$ & $r$ & $\begin{array}{l}n \\
a \\
\text { n }\end{array}$ & $\begin{array}{l}\infty \\
\text { i }\end{array}$ & $\begin{array}{l}a \\
\dot{\gamma} \\
\hat{i}\end{array}$ & $\begin{array}{l}0 \\
\text { i }\end{array}$ & $\hat{N}$ & $\sim$ \\
\hline $\begin{array}{l}0 \\
0 \\
\dot{0} \\
\dot{0}\end{array}$ & & 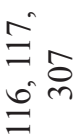 & $\frac{N}{m}$ & & in & $g$ & $\bar{n}$ & in & $n$ & $n$ & $\stackrel{+}{n}$ & $\frac{\vartheta}{\gamma}$ \\
\hline 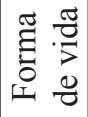 & & $\dot{z}$ & 文 & & $I$ & $I$ & $\Psi$ & $I$ & $I$ & $\Psi$ & $I$ & 完 \\
\hline 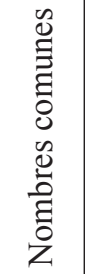 & & 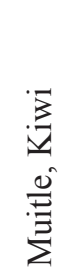 & 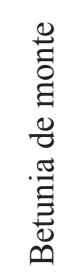 & & 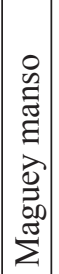 & 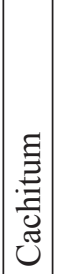 & 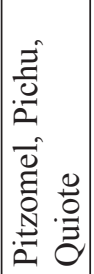 & 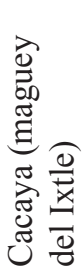 & 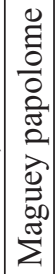 & 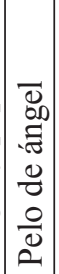 & 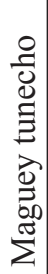 & 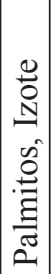 \\
\hline 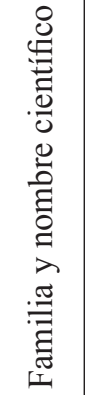 & 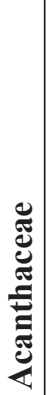 & 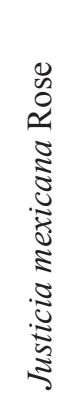 & 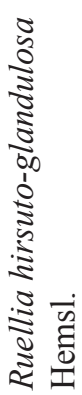 & 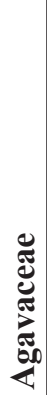 & 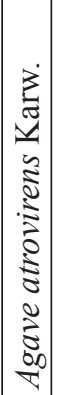 & 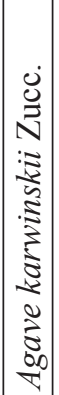 & 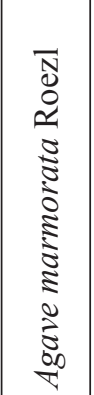 & 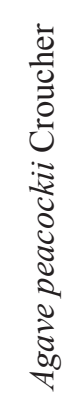 & 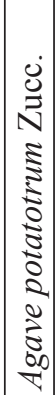 & 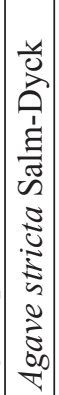 & $\begin{array}{l}\dot{2} \\
\dot{2} \\
0 \\
\vec{z} \\
0 \\
\nabla\end{array}$ & 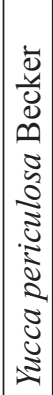 \\
\hline
\end{tabular}




\begin{tabular}{|c|c|c|c|c|c|c|c|c|c|c|c|c|c|c|c|c|}
\hline 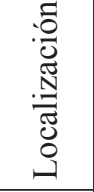 & & 壱 & & 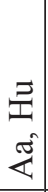 & 至 & 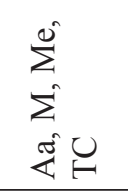 & & त) & 辛 & 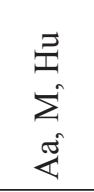 & $\underset{\Xi}{\vec{I}}$ & $\sum_{\Sigma}^{0}$ & & $\underset{\Xi}{\Xi}$ & & 吾 \\
\hline 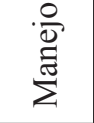 & & $\vec{U}$ & & $\ominus$ & $\vec{U}$ & $\bar{\sim}$ & & $\bar{\sim}$ & $\vec{U}$ & $\ominus$ & $\vec{U}$ & $\ddot{\sim}$ & & $\Xi$ & & تُ \\
\hline 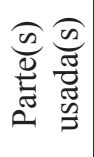 & & $I$ & & $I$ & a & 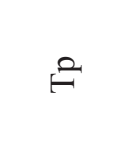 & & 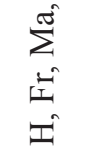 & 它 & $\frac{N}{\sum}$ & 它 & $\sum^{\pi}$ & & $\begin{array}{l}2 \\
-1 \\
-1\end{array}$ & & $\stackrel{\tilde{E}}{\underline{I}}$ \\
\hline $\begin{array}{l}n \\
0 \\
\mathscr{D}\end{array}$ & & 6 & & $\stackrel{\sim}{\sim}$ & 0 & $\stackrel{+}{\sim}$ & & $\hat{\sim} \dot{\sim}$ & $\sim$ & $\begin{array}{l}\dot{\nabla} \pm \\
\text { ñ } \\
\text { n }\end{array}$ & $\mathrm{N}$ & $\stackrel{ }{ }$ & & $\begin{array}{l} \pm \\
\text { त }\end{array}$ & & in \\
\hline $\begin{array}{ll}0 & \frac{\pi}{0} \\
\dot{0} & \frac{0}{0} \\
Z & 0\end{array}$ & & $\stackrel{\infty}{\stackrel{\infty}{N}}$ & & $\begin{array}{l}\approx \\
\\
\check{a}\end{array}$ & in & 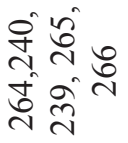 & & $\stackrel{\infty}{n}$ & in & 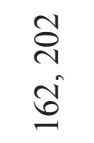 & ஜ & $\vec{ర}$ & & ले & & ชె \\
\hline 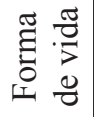 & & $I$ & & $I$ & $I$ & $I$ & & $\ll$ & $\varangle$ & $\ll$ & $\varangle$ & $\ll$ & & $\varangle$ & & $I$ \\
\hline 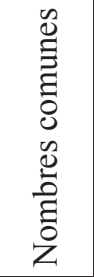 & & 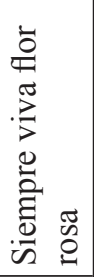 & & :气 & \begin{tabular}{|c|}
$\frac{\pi}{3}$ \\
$\tilde{\pi}$ \\
0 \\
$\Sigma$ \\
\end{tabular} & 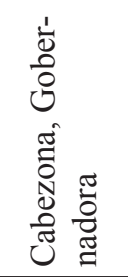 & & 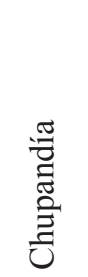 & $\begin{array}{l}\stackrel{0}{00} \\
\stackrel{\Xi}{E}\end{array}$ & 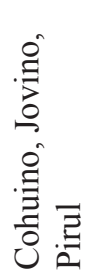 & 离 & $\frac{0}{\frac{E}{0}}$ & & 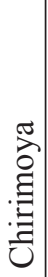 & & $\begin{array}{l}\stackrel{0}{\Xi} \\
\stackrel{\Xi}{\Xi} \\
\stackrel{\Xi}{U}\end{array}$ \\
\hline 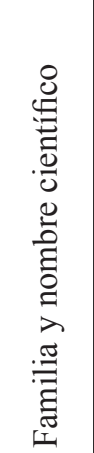 & 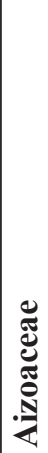 & 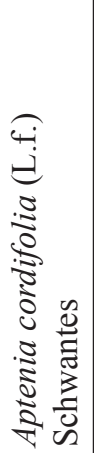 & 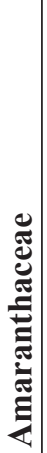 & 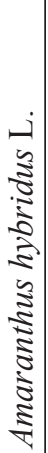 & 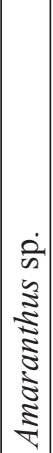 & 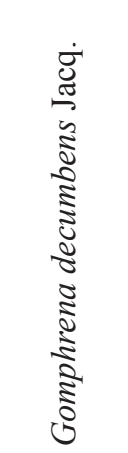 & 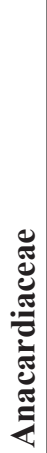 & 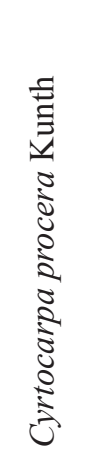 & 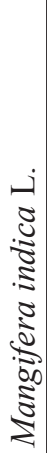 & 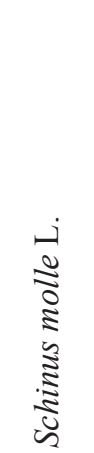 & 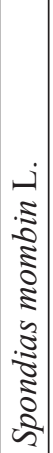 & 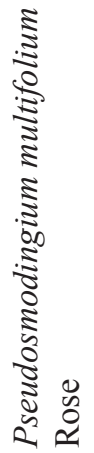 & 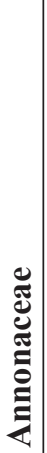 & 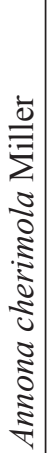 & $\begin{array}{l} \\
\\
\frac{\pi}{\pi} \\
\frac{\pi}{2} \\
\frac{\pi}{2}\end{array}$ & $\begin{array}{c}\dot{1} \\
\vdots \\
\vdots \\
\vdots \\
0 \\
0 \\
\vdots \\
\vdots \\
\vdots \\
\vdots \\
\vdots \\
\vdots \\
\vdots \\
0 \\
0 \\
0\end{array}$ \\
\hline
\end{tabular}


Paredes-Flores et al.: Estudio etnobotánico de Zapotitlán Salinas, Puebla

\begin{tabular}{|c|c|c|c|c|c|c|c|c|c|c|c|c|c|c|c|c|c|c|c|c|}
\hline 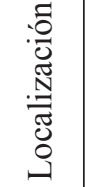 & ت્ચ & $\Xi$ & & 至 & $\mid \begin{array}{l}\vec{\Xi} \\
\tilde{\theta} \\
\hat{\theta} \\
\hat{n} \\
\tilde{D}\end{array}$ & $\frac{\vec{I}}{\Sigma}$ & & 寻 & 声 & $\underline{\Xi}$ & $\vec{\Xi}$ & 至 & 吾 & $\Xi$ & & $\begin{array}{l}\vec{\Xi} \\
\tilde{\Xi}\end{array}$ & $\begin{array}{l}\vec{\Xi} \\
\text { E⿱一兀 }\end{array}$ & $\Xi$ & $\Xi$ & 寻 \\
\hline $\begin{array}{l}\frac{0}{\mathbb{E}} \\
\stackrel{\Xi}{\Sigma}\end{array}$ & تُ & $\tilde{U}$ & & $\vec{U}$ & $\vec{\omega}$ & $\bar{\sim}$ & & $\Xi$ & $\vec{U}$ & تే & $\vec{U}$ & $\vec{\Xi}$ & $\vec{U}$ & $\vec{U}$ & & $\bar{\sim}$ & $\bar{\sim}$ & $\vec{\Xi}$ & تే & تَ \\
\hline 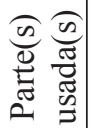 & $\stackrel{\pi}{=}$ & $I$ & & $\begin{array}{l}\vec{I} \\
\vec{I}\end{array}$ & $\overrightarrow{\mid \vec{I}}$ & 童 & & $\begin{array}{l}\vec{I} \\
\underline{I}\end{array}$ & $\Psi$ & $I$ & $I$ & $\Psi$ & $I$ & $\begin{array}{l}\vec{I} \\
\vec{I}\end{array}$ & & $I$ & $\begin{array}{l}\hat{E} \\
\underline{I}\end{array}$ & $\mid \begin{array}{l}0 \\
\hat{-1} \\
:\end{array}$ & $I$ & $I$ \\
\hline $\begin{array}{l}\mathscr{0} \\
0 \\
\emptyset\end{array}$ & $\nabla$ & n & & $\begin{array}{l}0 \\
0 \\
0\end{array}$ & 0 & $\begin{array}{l}0 \\
\text { i }\end{array}$ & & 0 & 6 & 6 & 0 & 6 & 6 & 6 & & $\begin{array}{l}\exists \\
\sigma^{\circ} \\
\dot{\nabla}\end{array}$ & 6 & $\begin{array}{l}0 \\
\text { in }\end{array}$ & 6 & 6 \\
\hline \begin{tabular}{ll}
0 & \multirow{J}{0}{} \\
$\dot{0}$ & $\frac{0}{0}$ \\
$Z$ & 0
\end{tabular} & O & $\overline{6}$ & & กิ & $\tilde{6}$ & $\begin{array}{l}\infty \\
\infty \\
\infty \\
\infty \\
\infty \\
\infty\end{array}$ & & $\infty$ & $\hat{6}$ & ติ & $尺$ & t & 6 & ๑ & & $\stackrel{\overbrace{}}{\underset{\gamma}{\sim}}$ & $\underset{\sim}{\stackrel{\sim}{f}}$ & $\stackrel{\sim}{\underset{f}{\sim}}$ & $\stackrel{2}{\Im}$ & 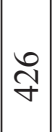 \\
\hline 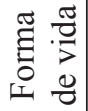 & $I$ & $I$ & & $\dot{z}$ & 文 & 交 & & $I$ & $I$ & $I$ & $\Psi$ & $\Psi$ & $I$ & $I$ & & $\varangle$ & $\ll$ & $\varangle$ & 交 & 去 \\
\hline 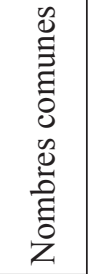 & $\frac{\circ}{\circ}$ & : & & $\frac{\pi}{\frac{\pi}{2}}$ & 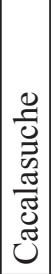 & 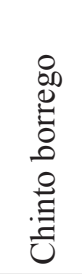 & & 呈 & $\begin{array}{l}\frac{\pi}{0} \\
\frac{\pi}{\pi} \\
\frac{\pi}{0} \\
\sum\end{array}$ & $\begin{array}{l}0 \\
. \\
0 \\
0 \\
0 \\
0 \\
0 \\
0 \\
0 \\
0 \\
0 \\
0 \\
0\end{array}$ & 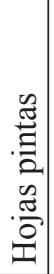 & 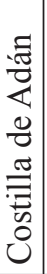 & \begin{tabular}{|c} 
\\
0 \\
0 \\
0 \\
$\frac{0}{0}$ \\
$\frac{0}{0}$ \\
0 \\
\end{tabular} & 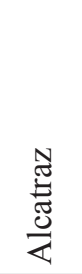 & & \begin{tabular}{|l|}
$\frac{\pi}{\Xi}$ \\
$\frac{\tilde{\sigma}}{\tilde{\sigma}}$ \\
\end{tabular} & בే & 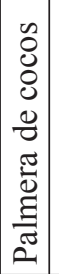 & 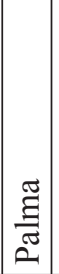 & $\frac{\widetilde{\sigma}}{\Xi}$ \\
\hline 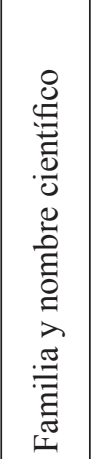 & 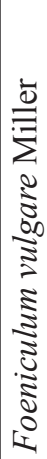 & 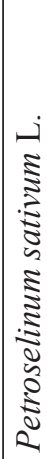 & 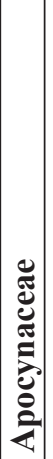 & 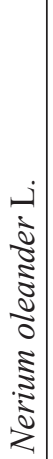 & 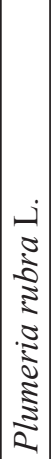 & 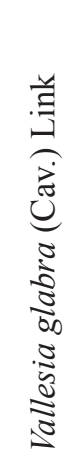 & 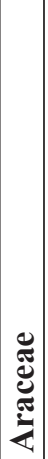 & 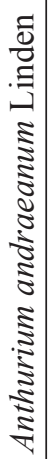 & 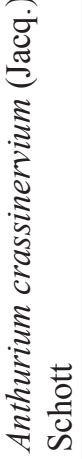 & 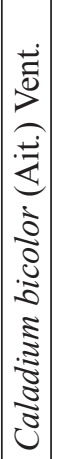 & 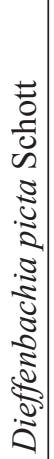 & 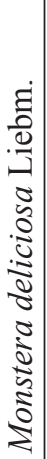 & 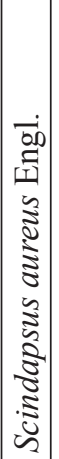 & 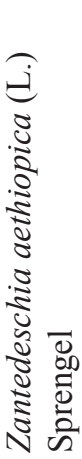 & 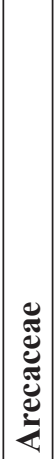 & 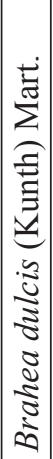 & 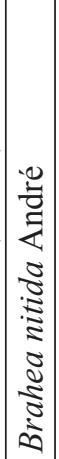 & $\mid \begin{array}{c}\mid \\
0 \\
0 \\
0 \\
0 \\
0 \\
0 \\
3 \\
0 \\
0 \\
0 \\
0 \\
0 \\
0\end{array}$ & $\mid$\begin{tabular}{c|}
0 \\
0 \\
0 \\
0 \\
0 \\
$\vdots$ \\
0 \\
0 \\
0 \\
0 \\
0 \\
0 \\
0 \\
0 \\
0 \\
0 \\
0 \\
0 \\
0
\end{tabular} & 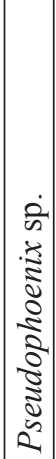 \\
\hline
\end{tabular}




\begin{tabular}{|c|c|c|c|c|c|c|c|c|c|c|c|c|c|c|c|c|}
\hline 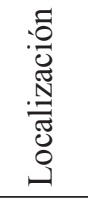 & & 章 & & 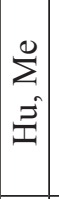 & & $\Xi$ & 录 & $\sum^{0}$ & 至 & $\Xi$ & $\Xi$ & $\begin{array}{l}\overrightarrow{1} \\
\text { లే }\end{array}$ & 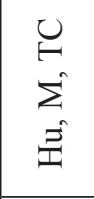 & 己 & $\begin{array}{l}\sum^{0} \\
\Xi \\
E\end{array}$ & $\begin{array}{l}\sum \\
\underline{\Xi}\end{array}$ \\
\hline 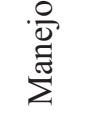 & & تُ & & $\vec{U}$ & & $\vec{U}$ & $\vec{U}$ & $\ddot{\sim}$ & $\vec{U}$ & $\vec{U}$ & $\vec{U}$ & $\stackrel{A}{i n}$ & $\bar{\sim}$ & $\bar{\sim}$ & $\bar{\sim}$ & $\bar{\omega}$ \\
\hline 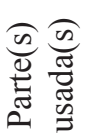 & & $\begin{array}{l}\text { 厌 } \\
\stackrel{\tilde{E}}{\oplus}\end{array}$ & & $\begin{array}{l}\tilde{\Xi} \\
\vec{\Xi} \\
\tilde{I}\end{array}$ & & $\begin{array}{l}\tilde{\sigma} \\
\tilde{I} \\
\tilde{I}\end{array}$ & $\stackrel{\widetilde{\pi}}{\stackrel{\tilde{I}}{\tilde{I}}}$ & $\stackrel{2}{F}$ & 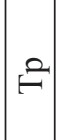 & 厌 & 厌 & $\stackrel{\vec{I}}{\vec{I}}$ & 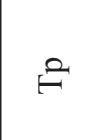 & 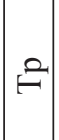 & $\begin{array}{l}\text { I } \\
\vec{I} \\
\tilde{I}\end{array}$ & $\frac{\vec{q}}{\vec{c}}$ \\
\hline $\begin{array}{l}\mathscr{n} \\
0 \\
\emptyset\end{array}$ & & 6 & & $\begin{array}{l}0 \\
\nabla^{2}\end{array}$ & & $\nabla$ & $\nabla$ & $\nabla$ & $\nabla$ & 0 & 6 & $\nabla$ & $\nabla$ & $\stackrel{+}{\sim}$ & $\stackrel{\nabla}{\stackrel{\sigma}{-}}$ & $\stackrel{\nabla}{\sim}$ \\
\hline $\begin{array}{ll}0 & \text { 总 } \\
\dot{0} & \frac{0}{0} \\
Z & 0\end{array}$ & & $\nabla$ & & 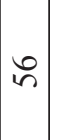 & & $\infty$ & $\stackrel{\Re}{\Xi}$ & 2 & 导 & 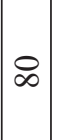 & ○ & $\cong$ & ๙ั & $\stackrel{m}{\sim}$ & 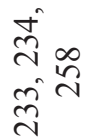 & 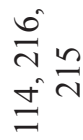 \\
\hline 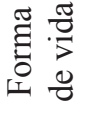 & & $I$ & & $I$ & & $I$ & $I$ & Z & $I$ & $I$ & 定 & $I$ & 完 & $I$ & 完 & 文 \\
\hline 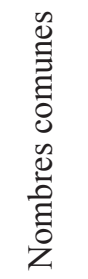 & & 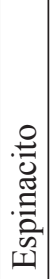 & & \begin{tabular}{l|}
$\frac{\pi}{3}$ \\
$\frac{\vec{\pi}}{\tilde{D}}$ \\
$\tilde{n}$
\end{tabular} & & 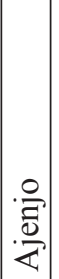 & 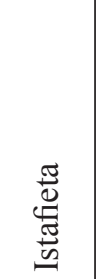 & 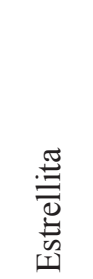 & $\mid \begin{array}{l}\frac{\pi}{0} \\
\frac{0}{0} \\
\frac{0}{0} \\
\frac{0}{0} \\
\Sigma\end{array}$ & 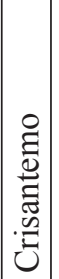 & 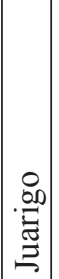 & $\frac{\stackrel{0}{:}}{\stackrel{0}{0}}$ & 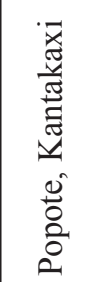 & . & 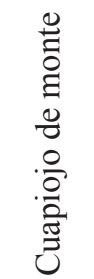 & $\frac{\frac{0}{0}}{\frac{0}{0}}$ \\
\hline 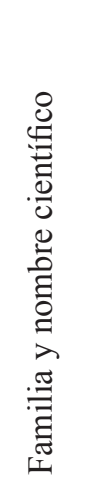 & 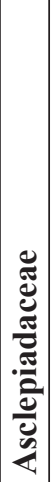 & 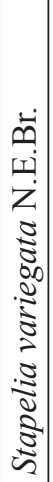 & 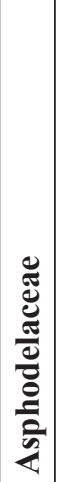 & 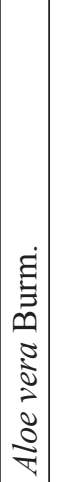 & . & 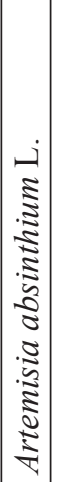 & 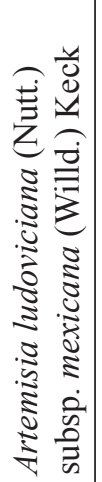 & 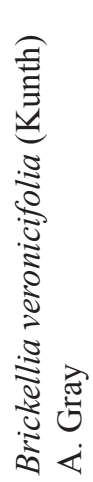 & 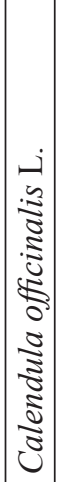 & 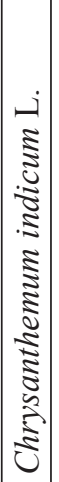 & $\mid \begin{array}{c} \\
\dot{2} \\
\frac{2}{2} \\
\frac{2}{2} \\
0 \\
0\end{array}$ & 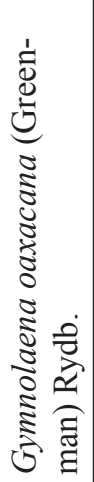 & 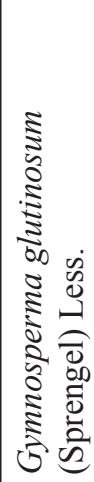 & 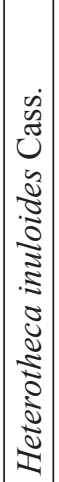 & 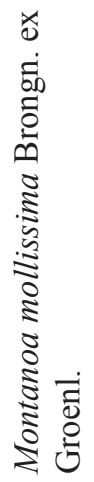 & 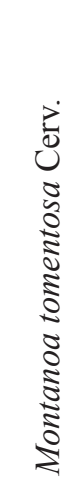 \\
\hline
\end{tabular}


Paredes-Flores et al.: Estudio etnobotánico de Zapotitlán Salinas, Puebla

\begin{tabular}{|c|c|c|c|c|c|c|c|c|c|c|c|c|c|c|c|c|c|}
\hline 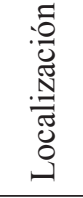 & $\begin{array}{l}\vec{\Xi} \\
\Sigma \\
\Sigma\end{array}$ & U & 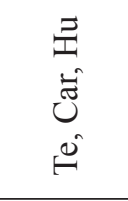 & $\begin{array}{l}\sum \\
\tilde{e} \\
\hat{E} \\
\hat{E}\end{array}$ & $\begin{array}{l}\stackrel{0}{4} \\
\text { 全 }\end{array}$ & 吾 & 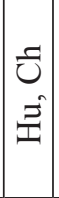 & 寻 & $\underset{\Xi}{\Xi}$ & $\stackrel{\pi}{\psi}$ & $\begin{array}{l}\Sigma \\
\underline{\Xi} \\
\underline{\Xi}\end{array}$ & $\begin{array}{l}\Sigma \\
\tilde{E} \\
\underline{\Xi}\end{array}$ & & $\underset{\Xi}{\Xi}$ & & $\underline{\Xi}$ & $\Xi$ \\
\hline$\frac{.}{\stackrel{\Xi}{\Xi}}$ & $\bar{\sim}$ & $\begin{array}{l}0 \\
i \\
i n\end{array}$ & $\bar{n}$ & $\bar{\sim}$ & $\bar{\omega}$ & $\vec{U}$ & $\begin{array}{l}0 \\
\dot{I} \\
\dot{\omega n}\end{array}$ & $\vec{J}$ & $\vec{\sim}$ & $\bar{\sim}$ & $\bar{\omega}$ & $\begin{array}{l}\vec{\oplus} \\
\vec{\omega}\end{array}$ & & $\vec{J}$ & & تُ & 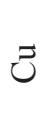 \\
\hline 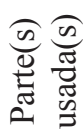 & 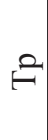 & $I$ & 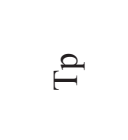 & $\stackrel{N}{\tilde{N}}$ & 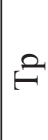 & 厌 & 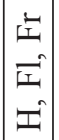 & $\begin{array}{l}\overrightarrow{\mid c} \\
\vec{I} \\
\vec{I}\end{array}$ & 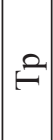 & $I$ & $I$ & $I$ & & $\begin{array}{l}\vec{I} \\
\underline{I}\end{array}$ & & $\begin{array}{l}\vec{I} \\
I^{\prime}\end{array}$ & $\underline{I}$ \\
\hline $\begin{array}{l}\tilde{n} \\
\stackrel{\infty}{\infty}\end{array}$ & $\begin{array}{l}\nabla \\
-\end{array}$ & $\stackrel{\sim}{\sim}$ & $\stackrel{+}{\sim}$ & $\stackrel{+}{\sim}$ & $\stackrel{\nabla}{\sim}$ & $\frac{m}{0}$ & 0 & $\nabla$ & $\nabla$ & $\begin{array}{l}\nabla \\
\stackrel{-}{*}\end{array}$ & - & $\begin{array}{l}0 \\
+\end{array}$ & & 0 & & 0 & 0 \\
\hline $\begin{array}{l}\frac{\pi}{0} \\
\dot{0} \\
\dot{0}\end{array}$ & $\cong$ & $\mathbb{N}$ & 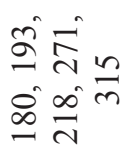 & ৪্ & $\underset{\sim}{\sim}$ & $\bar{\sim}$ & $\stackrel{n}{\sim}$ & $\underset{⿱}{\mathbb{N}}$ & $n$ & 12 & $\begin{array}{l}\stackrel{\tilde{r}}{+} \\
\vec{\sim} \\
\stackrel{\sim}{\sim} \\
\widetilde{v}\end{array}$ & $\approx$ & & $\stackrel{n}{=}$ & & $\vec{\infty}$ & $\infty$ \\
\hline 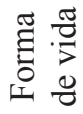 & $I$ & $I$ & $\Psi$ & 文 & $I$ & $I$ & $I$ & 完 & $I$ & 文 & $I$ & 完 & & $I$ & & $I$ & $I$ \\
\hline 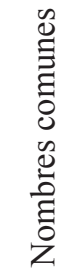 & 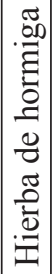 & $\begin{array}{l}0 \\
0 \\
0 \\
0 \\
0 \\
00 \\
0 \\
0 \\
0\end{array}$ & 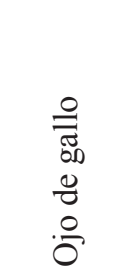 & 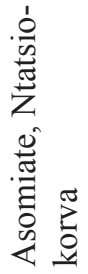 & 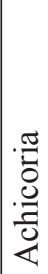 & 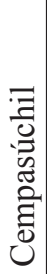 & 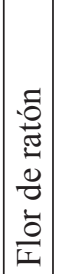 & 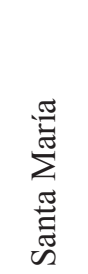 & \begin{tabular}{|c|}
$\stackrel{0}{0}$ \\
$\frac{0}{0}$ \\
0 \\
0 \\
0 \\
$\stackrel{0}{0}$ \\
$\stackrel{0}{0}$ \\
.0
\end{tabular} & 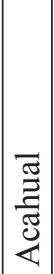 & 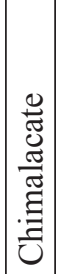 & 을 & & 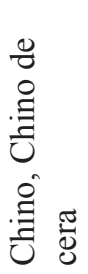 & & 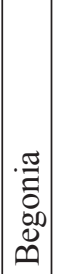 & 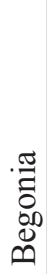 \\
\hline 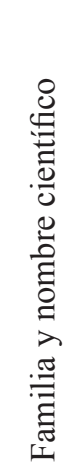 & 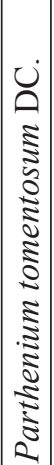 & 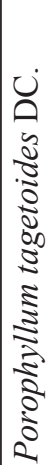 & 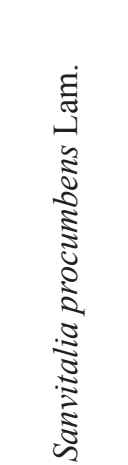 & 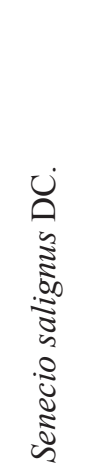 & 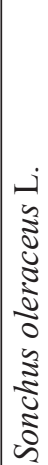 & 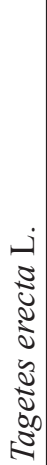 & $\begin{array}{c}0 \\
\dot{2} \\
2 \\
0 \\
0 \\
0 \\
0 \\
0\end{array}$ & 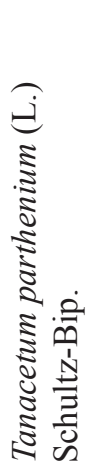 & 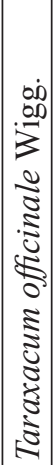 & 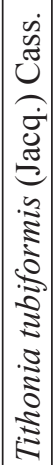 & 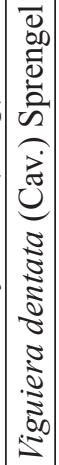 & 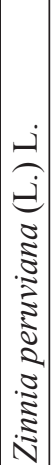 & 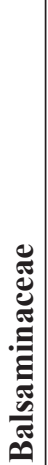 & 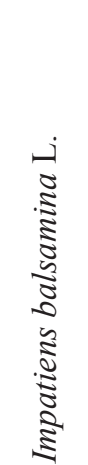 & 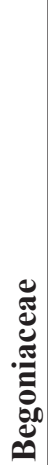 & 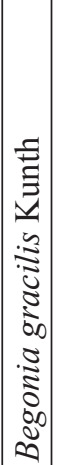 & 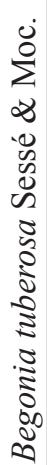 \\
\hline
\end{tabular}




\begin{tabular}{|c|c|c|c|c|c|c|c|c|c|c|c|c|c|c|c|c|c|}
\hline 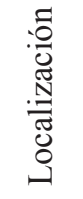 & & 吾 & 至 & 寻 & $\begin{array}{l}\sum \\
\tilde{0} \\
\tilde{E} \\
\tilde{E}\end{array}$ & & $\begin{array}{l}\overrightarrow{1} \\
\tilde{n} \\
\tilde{n}\end{array}$ & & $\sum^{0}$ & $\begin{array}{l}\Xi \\
\Sigma\end{array}$ & & 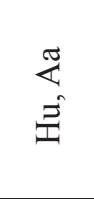 & 咅 & & $\stackrel{0}{\Sigma}$ & $\sum_{i}^{0}$ & $\frac{0}{2}$ \\
\hline$\frac{. \circ}{\stackrel{\Xi}{\Xi}}$ & & $\vec{U}$ & $\vec{U}$ & $\vec{U}$ & $\overrightarrow{i n}$ & & $\bar{\sim}$ & & $\ddot{\sim}$ & $\bar{\sim}$ & & $\bar{\Delta}$ & $\vec{U}$ & & $\bar{\sim}$ & $\ddot{\omega}$ & $\ddot{\sim}$ \\
\hline 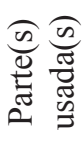 & & 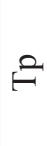 & 宅 & $\stackrel{2}{f}$ & $\vec{I}$ & & $\bar{I}$ & & $\begin{array}{l}\tilde{N} \\
\tilde{E} \\
\tilde{E}\end{array}$ & F & & $\hat{F}$ & $\stackrel{2}{E}$ & & $I$ & $\stackrel{\varrho}{\ominus}$ & $\stackrel{?}{\oplus}$ \\
\hline $\begin{array}{l}n \\
0 \\
\infty \\
\emptyset\end{array}$ & & $\begin{array}{l}\Xi \\
\sigma \\
m\end{array}$ & $\begin{array}{l}0 \\
2 \\
-1\end{array}$ & 0 & $\begin{array}{l}0 \\
-\end{array}$ & & $\begin{array}{l}n \\
i \\
-\end{array}$ & & - & $\nabla$ & & $\nabla$ & $\begin{array}{l}6 \\
7\end{array}$ & & - & $\stackrel{\nabla}{-}$ & - \\
\hline $\begin{array}{l}0 \\
\ddot{0} \\
\dot{0} \\
\dot{0}\end{array}$ & & $\infty$ & $\begin{array}{l}\hat{6} \\
\sim\end{array}$ & $\stackrel{\overbrace{}}{\beth}$ & Ð & & ‡ & & 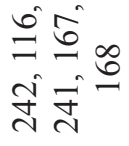 & 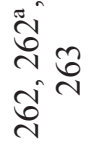 & & ๙ิ & $\stackrel{n}{n}$ & & $\mathscr{\infty}$ & $\begin{array}{l}\overline{0} \\
0 \\
0 \\
0 \\
0\end{array}$ & $\infty$ \\
\hline 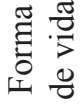 & & $\varangle$ & $\varangle$ & 交 & 安 & & $\varangle$ & & 宗 & 安 & & $I$ & $I$ & & $I$ & $\Psi$ & $I$ \\
\hline 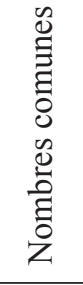 & & 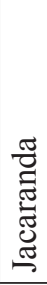 & : & 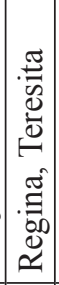 & 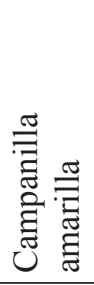 & & \begin{tabular}{|l|}
0 \\
0 \\
0 \\
0 \\
0 \\
0 \\
\end{tabular} & & 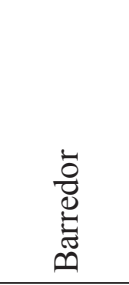 & 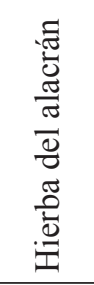 & & 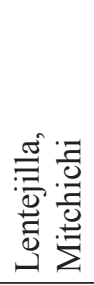 & 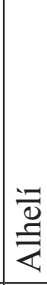 & & 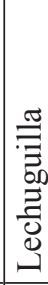 & 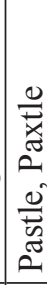 & 尊 \\
\hline 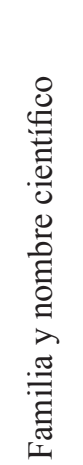 & 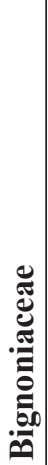 & 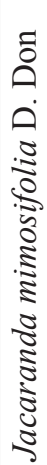 & 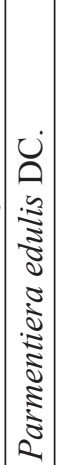 & 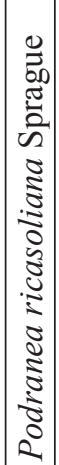 & 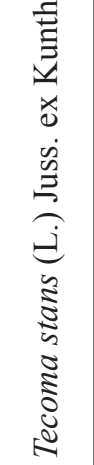 & 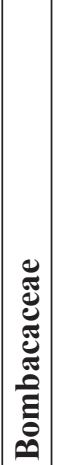 & $\mid$\begin{tabular}{c}
0 \\
0 \\
0 \\
2 \\
0 \\
$: 0$ \\
0 \\
$: 0$ \\
0 \\
0 \\
$\vdots$ \\
0 \\
0 \\
\hdashline 0 \\
0
\end{tabular} & 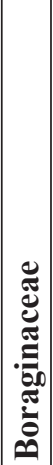 & 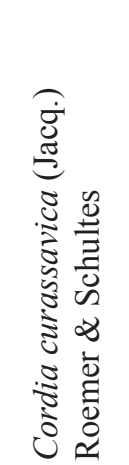 & 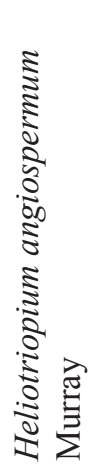 & 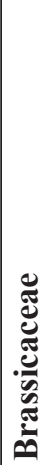 & 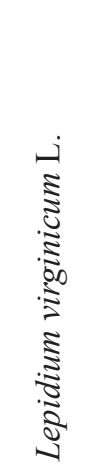 & 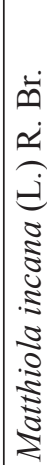 & مَّ & 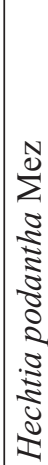 & 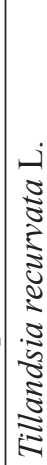 & 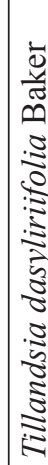 \\
\hline
\end{tabular}


Paredes-Flores et al.: Estudio etnobotánico de Zapotitlán Salinas, Puebla

\begin{tabular}{|c|c|c|c|c|c|c|c|c|c|c|c|c|c|}
\hline 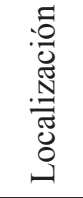 & & $\begin{array}{l}0 \\
\dot{\infty} \\
\dot{0}\end{array}$ & 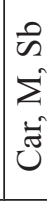 & & שี & 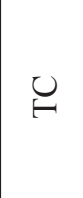 & 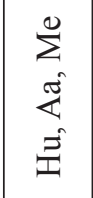 & $\begin{array}{l}\vec{I} \\
\sum^{\oplus} \\
\dot{e}^{n}\end{array}$ & $\begin{array}{l}\sum_{0}^{0} \\
\sum_{0}^{0}\end{array}$ & 吾 & 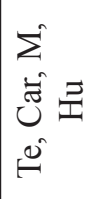 & 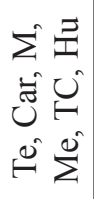 & 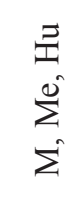 \\
\hline $\begin{array}{l}\frac{\circ}{\mathbb{E}} \\
\stackrel{\Xi}{\Sigma}\end{array}$ & & $\bar{\sim}$ & $\bar{\omega}$ & & $\bar{\omega}$ & $\bar{\sim}$ & $\bar{\sim}$ & $\bar{\omega}$ & $\bar{\sim}$ & $\vec{J}$ & $\vec{\omega}$ & $\hat{i n}$ & $\overrightarrow{\tilde{B}}$ \\
\hline 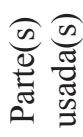 & & $\stackrel{\widetilde{\sigma}}{\oplus}$ & $\stackrel{\widetilde{\sigma}}{=}$ & & $\stackrel{\pi}{\stackrel{\pi}{=}}$ & $\stackrel{\overrightarrow{\mid c}}{\overrightarrow{I I}}$ & 空 & $\stackrel{\pi}{\simeq}$ & 新 & 空 & 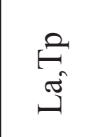 & $\stackrel{Q}{\theta}$ & $\sum_{i=1}^{\pi}$ \\
\hline $\begin{array}{l}\mathscr{n} \\
\varnothing \\
\wp\end{array}$ & & $\frac{\sim}{\sigma}$ & $\frac{\sim}{\sigma}$ & & $\sim$ & - & n & $\begin{array}{l}0 \\
\sim \\
\sim\end{array}$ & - & $\begin{array}{l}+ \\
\text { i }\end{array}$ & $\begin{array}{l}0 \\
\forall\end{array}$ & 6 & $\begin{array}{l}\stackrel{r}{a} \\
\stackrel{n}{n}\end{array}$ \\
\hline $\begin{array}{l}0 \\
\text { O } \\
\dot{0} \\
\dot{0} \\
\end{array}$ & & $\infty$ & 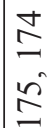 & & $\stackrel{n}{n}$ & $\infty$ & 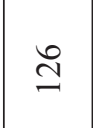 & ஓ & ஓ & $\bar{a}$ & ๙ૂ & a & ন \\
\hline 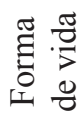 & & $\varangle$ & 运 & & $\dot{z}$ & $I$ & 交 & $I$ & $I$ & $I$ & $I$ & $I$ & $\varangle$ \\
\hline 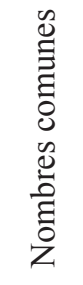 & & 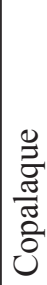 & 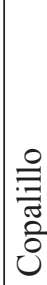 & & 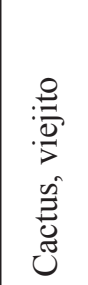 & 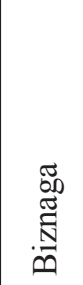 & $\stackrel{0}{\stackrel{0}{0}}$ & 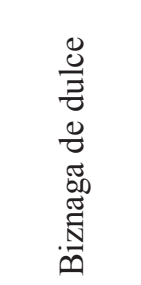 & 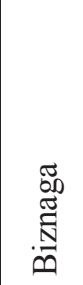 & 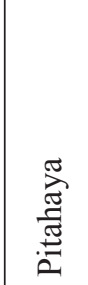 & 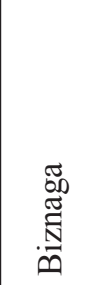 & 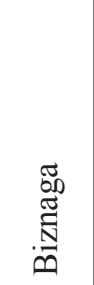 & 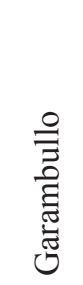 \\
\hline 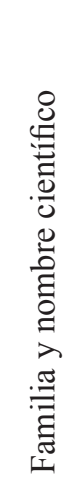 & 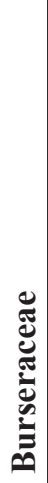 & 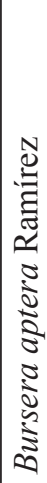 & 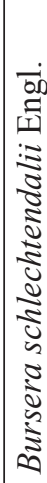 & 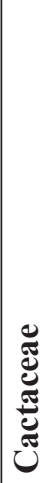 & 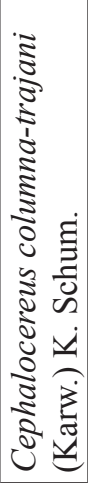 & 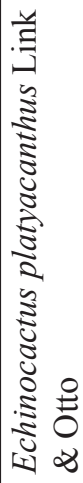 & 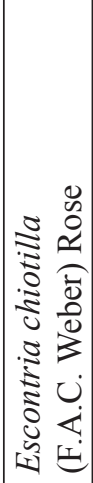 & 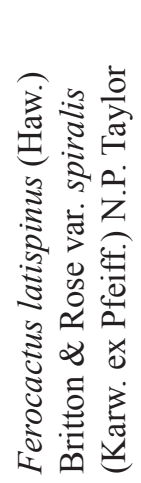 & 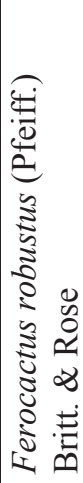 & 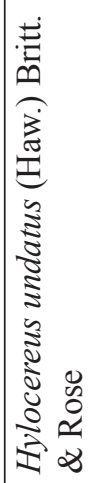 & 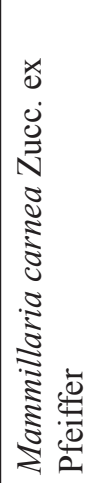 & 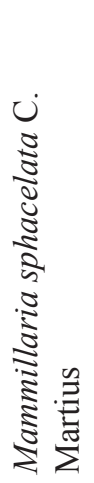 & 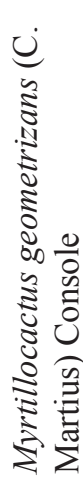 \\
\hline
\end{tabular}




\begin{tabular}{|c|c|c|c|c|c|c|c|c|c|c|c|c|c|}
\hline 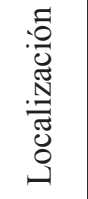 & 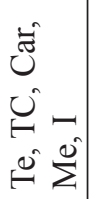 & $\underset{\Xi}{\Xi}$ & $\sum^{0}$ & 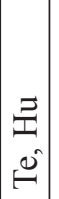 & 胥 & $\begin{array}{l}\sum_{0}^{\infty}-\tilde{U} \\
\sum_{0}^{0}-\end{array}$ & $\underset{\Xi}{\Xi}$ & 章 & $\sum^{0}$ & $\frac{\vec{I}}{\sum_{0}^{0}}$ & $\stackrel{\vec{I}}{\Sigma}$ & 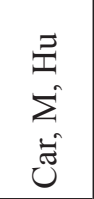 & $\sum^{0}$ \\
\hline$\frac{\stackrel{\circ}{\mathbb{E}}}{\sum}$ & 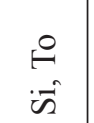 & $a$ & $\vec{n}$ & $\bar{\sim}$ & $\vec{U}$ & $\bar{\sim}$ & $\vec{\Xi}$ & $\vec{J}$ & $\dot{\sim}$ & 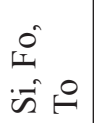 & $\begin{array}{l}0 \\
\dot{i n}\end{array}$ & $\bar{\omega}$ & $\bar{n}$ \\
\hline 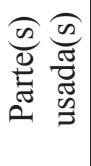 & 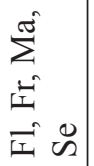 & $\frac{\overrightarrow{\mid c}}{\overrightarrow{\mid c}}$ & 它 & 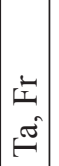 & 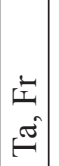 & $\stackrel{\pi}{\oplus}$ & \begin{tabular}{l} 
至 \\
\multirow{E}{*}{}
\end{tabular} & $\begin{array}{l}\text { 它 } \\
\text { है }\end{array}$ & $\stackrel{\widetilde{\pi}}{\oplus}$ & 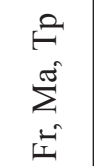 & $\begin{array}{l}\text { 空 } \\
\text { Eే }\end{array}$ & 榁 & $\begin{array}{l}\stackrel{\pi}{\rightleftarrows} \\
\stackrel{-1}{1}\end{array}$ \\
\hline $\begin{array}{l}n \\
0 \\
0 \\
\emptyset\end{array}$ & $\begin{array}{l}n \\
\tilde{r}\end{array}$ & - & $\begin{array}{l}+ \\
- \\
-\end{array}$ & $\sim$ & $\stackrel{\sim}{\sim}$ & $N$ & $N$ & $N$ & $\infty$ & $\begin{array}{l}n \\
i \\
i\end{array}$ & $\begin{array}{l}r \\
\dot{5} \\
\stackrel{-}{n}\end{array}$ & $\begin{array}{l}0 \\
\text { i }\end{array}$ & $\hat{n}$ \\
\hline \begin{tabular}{ll}
0 & \multirow{0}{0}{} \\
$\dot{0}$ & $\frac{0}{0}$ \\
$Z$ & 8
\end{tabular} & n & ஃ & $\hat{a}$ & $\begin{array}{l}1 \\
\infty \\
\infty \\
\infty \\
\infty\end{array}$ & $\stackrel{\beth}{\beth}$ & $\widehat{\beth}$ & $\underset{\Delta}{\Delta}$ & $\stackrel{\infty}{\sigma}$ & ๙ & 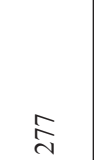 & $\stackrel{\widehat{\lambda}}{ }$ & $\frac{\sim}{m}$ & $\cong$ \\
\hline 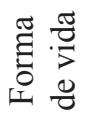 & $\ll$ & 交 & 文 & 运 & 完 & 交 & 文 & 完 & & 交 & Z & 完 & 文 \\
\hline 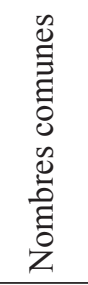 & 递 & 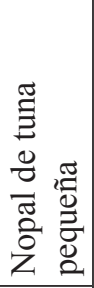 & $\mid \begin{array}{l}0 \\
0 \\
0 \\
0 \\
0 \\
0 \\
0 \\
\frac{0}{\pi} \\
0 \\
0 \\
z\end{array}$ & 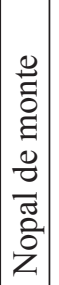 & 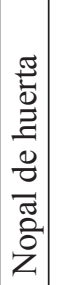 & 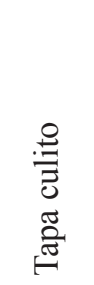 & 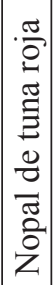 & 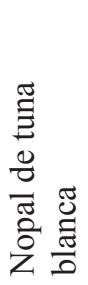 & $\begin{array}{l}0 \\
0 \\
0 \\
0 \\
\frac{0}{0} \\
0 \\
0 \\
0\end{array}$ & 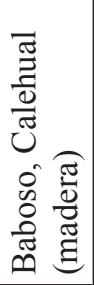 & $\begin{array}{l}0 \\
\frac{E}{0} \\
. \Xi \\
\sum^{\pi} \\
0 \\
0 \\
.00 \\
0 \\
0\end{array}$ & 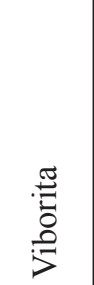 & 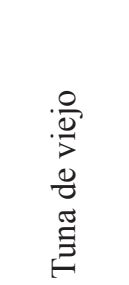 \\
\hline 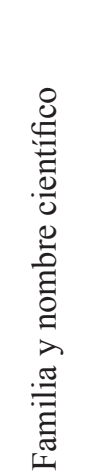 & 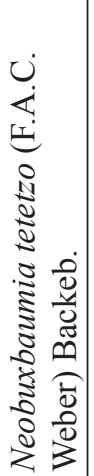 & 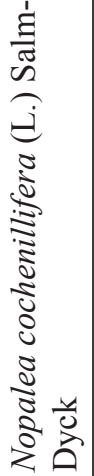 & 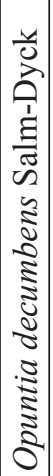 & 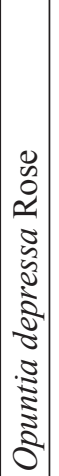 & 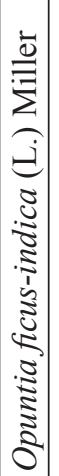 & 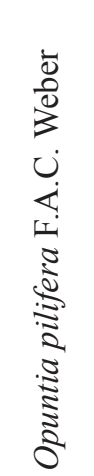 & 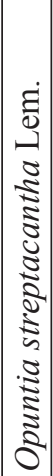 & 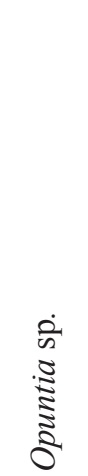 & 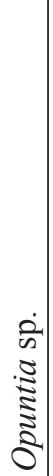 & 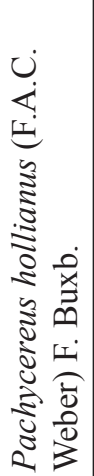 & 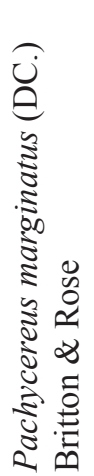 & 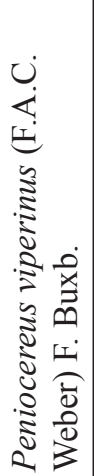 & 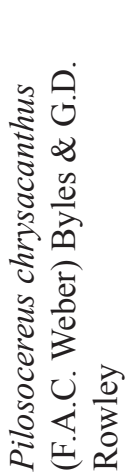 \\
\hline
\end{tabular}


Paredes-Flores et al.: Estudio etnobotánico de Zapotitlán Salinas, Puebla

\begin{tabular}{|c|c|c|c|c|c|c|c|c|c|c|c|c|c|c|c|}
\hline 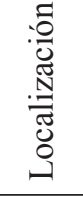 & 吾 & 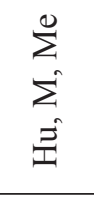 & & 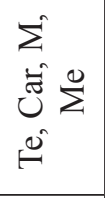 & $\underset{\Xi}{\Xi}$ & $\sum^{0}$ & 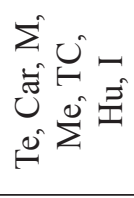 & $\sum^{0}$ & 忢 & & 吾 & & $\vec{\Xi}$ & & $\stackrel{\Xi}{\Xi}$ \\
\hline $\begin{array}{l}\stackrel{\circ}{\mathbb{E}} \\
\stackrel{\Xi}{\Sigma}\end{array}$ & $\begin{array}{l}0 \\
-1 \\
n\end{array}$ & $\begin{array}{l}\hat{\theta} \\
\dot{n} \\
\dot{n}\end{array}$ & & $\bar{\sim}$ & $\bar{\sim}$ & $\bar{\sim}$ & $\bar{\sim}$ & $\bar{\sim}$ & $\vec{~}$ & & $\vec{J}$ & & $\vec{U}$ & & $\vec{J}$ \\
\hline 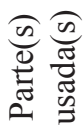 & $\sum_{i=1}^{\pi}$ & 空 & & $\stackrel{\pi}{\oplus}$ & $\begin{array}{l}\vec{I} \\
\vec{I}\end{array}$ & $I$ & $\stackrel{\pi}{\tilde{I}}$ & $\sum^{\pi}$ & 它 & & $\begin{array}{l}\vec{I} \\
I\end{array}$ & & $I$ & & 它 \\
\hline $\begin{array}{l}\mathscr{0} \\
0 \\
\infty \\
\infty\end{array}$ & $\begin{array}{l}n 0 \\
n-n\end{array}$ & $\begin{array}{l}\hat{r} \\
i\end{array}$ & & $\stackrel{n}{\rightarrow}$ & 6 & $\nabla$ & $\begin{array}{l}+ \\
\stackrel{2}{n}\end{array}$ & $\stackrel{m}{-}$ & $\begin{array}{l}\nabla \\
\text { i }\end{array}$ & & 6 & & $\nabla$ & & $\sim$ \\
\hline $\begin{array}{l}0 \\
0 \\
\dot{0} \\
\dot{0} \\
\end{array}$ & 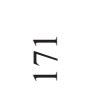 & $\stackrel{N}{I}$ & & $\hat{\overbrace{}}$ & $\begin{array}{l}\infty \\
\sim \\
\sim\end{array}$ & 하 & $\stackrel{\circ}{\circ}$ & 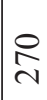 & $\stackrel{\infty}{\sim}$ & & 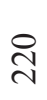 & & $\begin{array}{l}\infty \\
m \\
m\end{array}$ & & $\stackrel{n}{m}$ \\
\hline 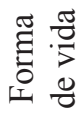 & 之 & 文 & & 文 & $\dot{z}$ & 完 & $\varangle$ & 文 & $\varangle$ & & $I$ & & $\varangle$ & & $I$ \\
\hline 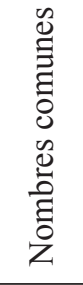 & 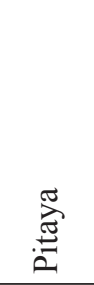 & $\begin{array}{l}0 \\
\stackrel{0}{0} \\
0 \\
0 \\
0 \\
0 \\
0\end{array}$ & & 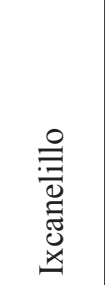 & $\begin{array}{l}0 \\
0 \\
0.00 \\
07 \\
0\end{array}$ & 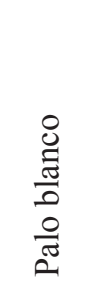 & 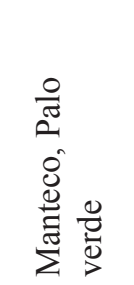 & 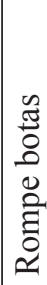 & 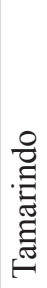 & & 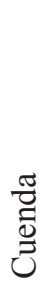 & & 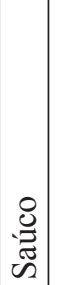 & & 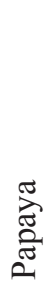 \\
\hline 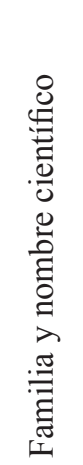 & 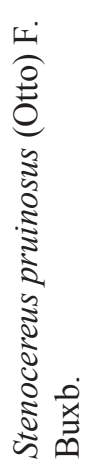 & 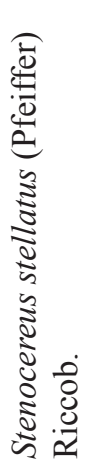 & 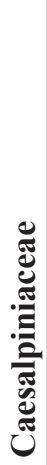 & 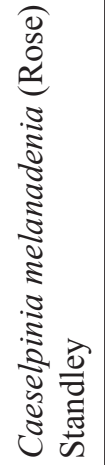 & 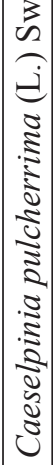 & 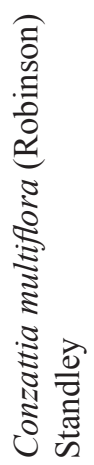 & 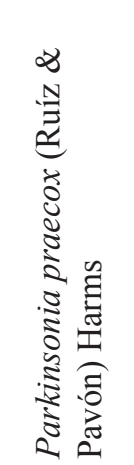 & $\begin{array}{l}\text { के } \\
\text { के } \\
\text { ह } \\
\text { के }\end{array}$ & 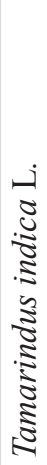 & 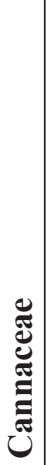 & 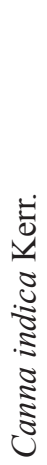 & 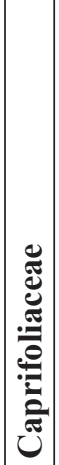 & 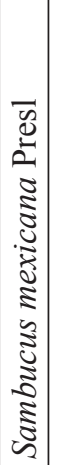 & ن. & 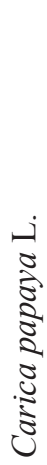 \\
\hline
\end{tabular}




\begin{tabular}{|c|c|c|c|c|c|c|c|c|c|c|c|c|c|c|c|c|c|c|}
\hline 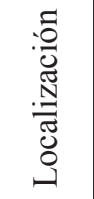 & & $\stackrel{\Xi}{\Xi}$ & & $\Xi$ & & $\Xi$ & 吾 & & $\Xi$ & 焉 & & 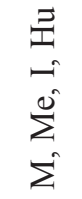 & & $\begin{array}{l}\tilde{U} \\
\hat{\Xi}\end{array}$ & $\underline{\Xi}$ & 吾 & $\begin{array}{l}\Sigma \\
\xi \\
\underline{\Xi}\end{array}$ & 夛 \\
\hline 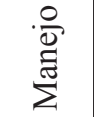 & & $\vec{U}$ & & $\vec{U}$ & & 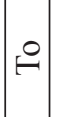 & $\begin{array}{l}0 \\
0 \\
0\end{array}$ & & $\vec{U}$ & $\vec{~}$ & & $\bar{\sim}$ & & $\begin{array}{l}\dot{\omega} \\
\dot{\omega}\end{array}$ & $\vec{U}$ & $\vec{J}$ & $\vec{U}$ & $\vec{~}$ \\
\hline 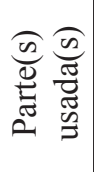 & & 可 & & $\Leftrightarrow$ & & 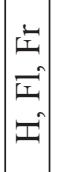 & $\begin{array}{l}\stackrel{\pi}{I} \\
\tilde{I}\end{array}$ & & $I$ & $I$ & & $\begin{array}{l}\stackrel{0}{E} \\
\sum_{\tilde{\Xi}}^{\tilde{\Xi}}\end{array}$ & & $\underline{E}$ & 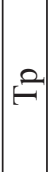 & 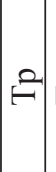 & a & $I$ \\
\hline $\begin{array}{l}\mathscr{0} \\
\mathscr{D} \\
\emptyset\end{array}$ & & 6 & & $\frac{\nabla}{0^{\circ}}$ & & $\begin{array}{l}+ \\
-\end{array}$ & $\begin{array}{l}\Xi \\
\stackrel{7}{\sigma}\end{array}$ & & $\nabla$ & $\nabla$ & & $\begin{array}{l}\sigma^{\circ} \\
\dot{f}^{\circ} \\
\overbrace{}^{2}\end{array}$ & & $\begin{array}{l}0 \\
+\end{array}$ & 0 & 0 & 0 & 0 \\
\hline $\begin{array}{ll}0 & \frac{\pi}{0} \\
\dot{0} & \frac{0}{0} \\
\dot{0}\end{array}$ & & $\stackrel{\hat{N}}{\sim}$ & & 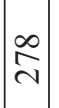 & & $\stackrel{\sim}{\simeq}$ & $\stackrel{ \pm}{n}$ & & $\stackrel{\infty}{=}$ & $\stackrel{0}{n}$ & & $\frac{\curvearrowright}{\lambda}$ & & $\vec{\infty}$ & $\left|\begin{array}{c}\mathcal{N} \\
\text { ஸे }\end{array}\right|$ & $\left|\begin{array}{c}0 \\
\infty \\
\sim\end{array}\right|$ & $\begin{array}{l}+ \\
\infty \\
\text { N }\end{array}$ & $\stackrel{n}{\infty}$ \\
\hline 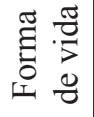 & & $I$ & & $\varangle$ & & $I$ & $I$ & & $I$ & $I$ & & 完 & & $I$ & $I$ & $I$ & $I$ & $I$ \\
\hline 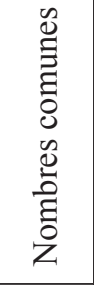 & & $\begin{array}{l}\overline{0} \\
\stackrel{0}{0} \\
\end{array}$ & & 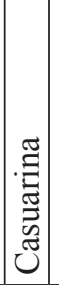 & & 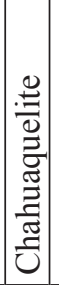 & 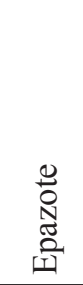 & & 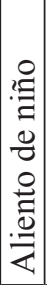 & $\stackrel{0}{\overline{0}}$ & & 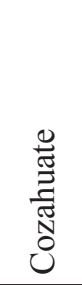 & & 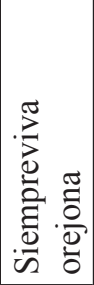 & 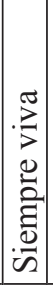 & 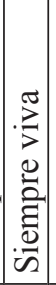 & 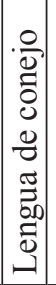 & 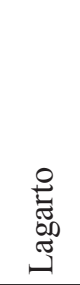 \\
\hline 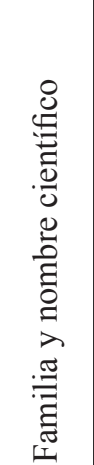 & 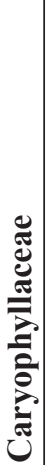 & 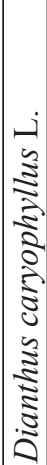 & 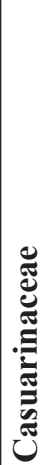 & 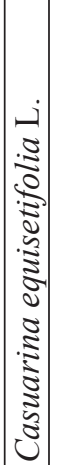 & 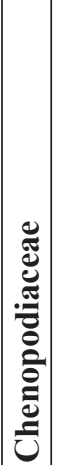 & $\mid$\begin{tabular}{|c|} 
\\
\\
1 \\
0 \\
0 \\
$\vdots$ \\
$\vdots$ \\
$\vdots$ \\
$\vdots$ \\
$\vdots$ \\
$\vdots$ \\
-3 \\
0 \\
$\vdots$ \\
$\vdots$ \\
0 \\
0 \\
0 \\
0
\end{tabular} & 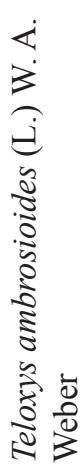 & 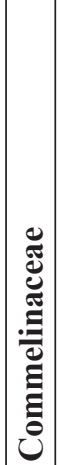 & 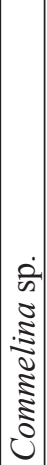 & 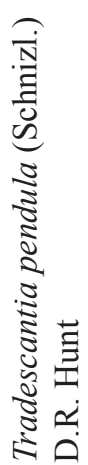 & 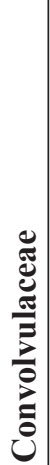 & 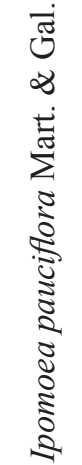 & 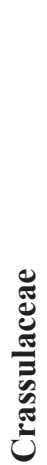 & $\begin{array}{c}0 \\
0 \\
0 \\
0 \\
0 \\
0 \\
0 \\
0 \\
00 \\
0 \\
0 \\
0 \\
0 \\
0 \\
0 \\
0 \\
0\end{array}$ & 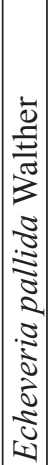 & 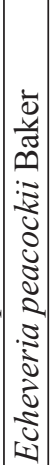 & 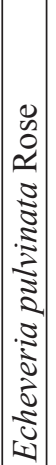 & 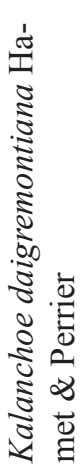 \\
\hline
\end{tabular}


Paredes-Flores et al.: Estudio etnobotánico de Zapotitlán Salinas, Puebla

\begin{tabular}{|c|c|c|c|c|c|c|c|c|c|c|c|c|c|c|c|c|c|}
\hline 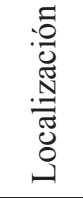 & 吾 & $\begin{array}{l}\vec{\Xi} \\
\dot{\Sigma}\end{array}$ & 吾 & $\stackrel{\Xi}{ \pm}$ & $\underset{\Xi}{\Xi}$ & 吾 & & $\frac{\pi}{4}$ & $\stackrel{\pi}{<}$ & $\begin{array}{l}\stackrel{\Xi}{I} \\
\underset{\mathbb{*}}{*}\end{array}$ & 忢 & $\stackrel{\pi}{4}$ & $\stackrel{\pi}{<}$ & 吾 & & $\underset{\Xi}{\Xi}$ & 寻 \\
\hline $\begin{array}{l}\stackrel{\circ}{\mathbb{D}} \\
\sum_{\Sigma}^{\mathbb{E}}\end{array}$ & $\vec{z}$ & $\begin{array}{l}\vec{H} \\
\vec{\omega}\end{array}$ & $\vec{U}$ & $\vec{J}$ & $\vec{J}$ & $\vec{~}$ & & $\begin{array}{l}\stackrel{0}{\ominus} \\
\dot{\omega}\end{array}$ & ت & $\vec{J}$ & $\vec{U}$ & تُ & $\vec{J}$ & $\vec{J}$ & & $\vec{U}$ & $\bar{J}$ \\
\hline 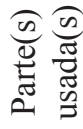 & $I$ & $\Psi$ & $I$ & E & $\stackrel{2}{n}$ & $\stackrel{2}{E}$ & & $\ddot{\sim}$ & 鳬 & 式 & 它 & 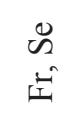 & $\begin{array}{l}\vec{I} \\
\vec{H}\end{array}$ & 式 & & $\stackrel{0}{E}$ & e \\
\hline $\begin{array}{l}\text { on } \\
0 \\
0\end{array}$ & 6 & $\begin{array}{c}0 \\
\nabla^{n}\end{array}$ & $\begin{array}{l}0 \\
+\end{array}$ & 6 & 6 & 6 & & $\sim$ & $N$ & $\sim$ & $\stackrel{\sim}{\sim}$ & $\stackrel{\sim}{\sim}$ & $\stackrel{\sim}{-}$ & $\sim$ & & 0 & 6 \\
\hline $\begin{array}{l}0 \\
0 \\
\dot{0} \\
\dot{0} \\
\end{array}$ & $\begin{array}{l}\infty \\
\text { d }\end{array}$ & $\underset{\sim}{\infty}$ & 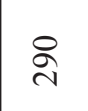 & $\begin{array}{l}\infty \\
\stackrel{\infty}{\sim}\end{array}$ & $\underset{\sim}{\stackrel{\infty}{\sim}}$ & $\overline{\widehat{\lambda}}$ & & $\hat{\curvearrowright}$ & ঐे & $\stackrel{2}{\curvearrowright}$ & $\bar{m}$ & $\stackrel{\infty}{\underset{N}{N}}$ & సิ & 守 & & તે & $\bar{\beth}$ \\
\hline 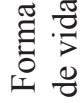 & $I$ & $\boldsymbol{I}$ & 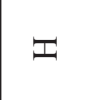 & $\Psi$ & $I$ & $I$ & & $\boldsymbol{I}$ & $I$ & $I$ & $I$ & $I$ & $I$ & I & & 完 & 宗 \\
\hline 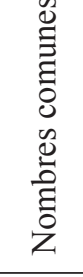 & 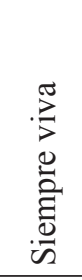 & 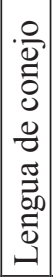 & 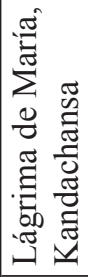 & 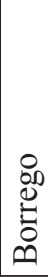 & 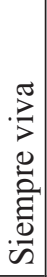 & 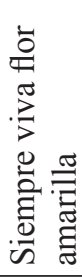 & & $\begin{array}{l}\stackrel{0}{\Xi} \\
\overline{0} \\
\frac{0}{0} \\
\stackrel{0}{\Sigma}\end{array}$ & 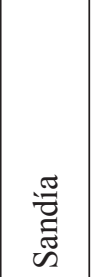 & $\frac{\Xi 0}{\sum^{0}}$ & 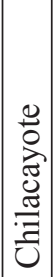 & 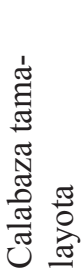 & 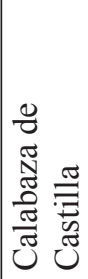 & 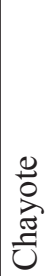 & & 这 & $\stackrel{\circ}{.}$ \\
\hline 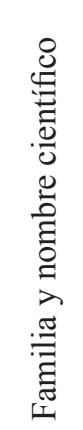 & 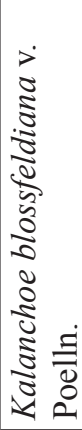 & 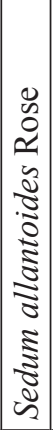 & 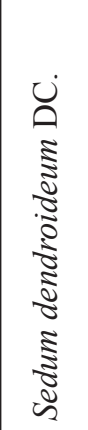 & 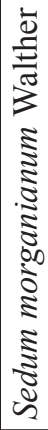 & 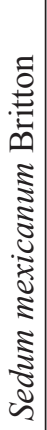 & 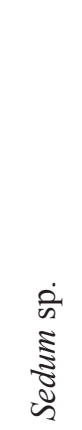 & 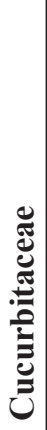 & 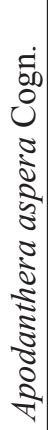 & 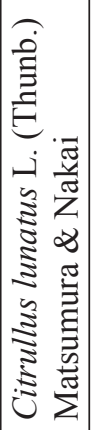 & 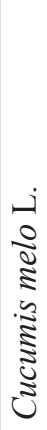 & 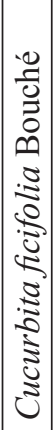 & 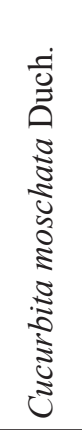 & 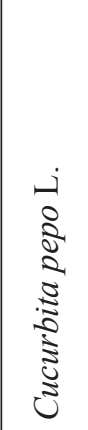 & 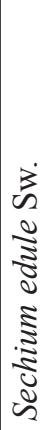 & 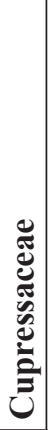 & 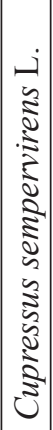 & : \\
\hline
\end{tabular}




\begin{tabular}{|c|c|c|c|c|c|c|c|c|c|c|c|c|c|c|c|c|}
\hline 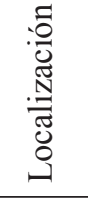 & & 茎 & & $\begin{array}{l}\frac{\pi}{4} \\
e^{\infty}\end{array}$ & $\underset{\Xi}{\vec{I}}$ & $\begin{array}{l}\overrightarrow{\mid} \\
\dot{\Sigma}\end{array}$ & 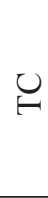 & $\Xi$ & 焉 & $\underset{I}{\Xi}$ & 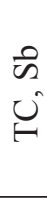 & 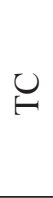 & 孚 & & $\begin{array}{l}\tilde{U} \\
\tilde{E}\end{array}$ & 妾 \\
\hline$\frac{\circ}{\stackrel{\circ}{\Xi}}$ & & ت & & $\bar{\sim}$ & $\ddot{\sim}$ & $\bar{\sim}$ & $\bar{D}$ & $\vec{U}$ & $\vec{U}$ & $\bar{\omega}$ & $\bar{\Delta}$ & $\bar{\sim}$ & $\bar{\omega}$ & & $\bar{s}$ & $\vec{U}$ \\
\hline 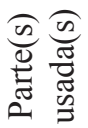 & & 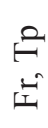 & & $\Leftrightarrow$ & $\begin{array}{l}\dot{I} \\
\vec{I}\end{array}$ & $\stackrel{\tilde{I}}{\tilde{I}}$ & $\stackrel{\widetilde{\sigma}}{\Xi}$ & $\stackrel{\pi}{=}$ & $\begin{array}{l}0 \\
I \\
I\end{array}$ & 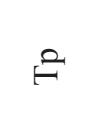 & $\stackrel{\widetilde{\sigma}}{\Xi}$ & $\stackrel{\widetilde{J}}{\Xi}$ & $\ddot{n}$ & & 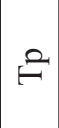 & $\stackrel{\pi}{=}$ \\
\hline $\begin{array}{l}n \\
0 \\
0 \\
n\end{array}$ & & $N$ & & $\nabla$ & $\begin{array}{l}+ \\
\text { i }\end{array}$ & $\nabla$ & $\cong$ & 0 & 6 & 6 & $\stackrel{\nabla}{\sim}$ & $\nabla$ & $\nabla$ & & 6 & - \\
\hline 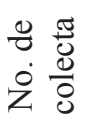 & & $\underset{\forall}{\stackrel{丶}{\forall}}$ & & $\begin{array}{l}\infty \\
\stackrel{2}{\Sigma} \\
\Sigma\end{array}$ & ๗ิ & $\tilde{6}$ & $\stackrel{2}{\partial}$ & ๖̊ & $\hat{n}$ & $\widetilde{\sigma}$ & $\stackrel{8}{0}$ & $\stackrel{\infty}{2}$ & $\tilde{N}$ & & $\stackrel{n}{\sim}$ & $\underset{\sim}{\stackrel{J}{\sim}}$ \\
\hline 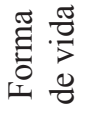 & & $\varangle$ & & $I$ & $I$ & $\Psi$ & 文 & Z & 完 & 安 & 安 & 安 & $I$ & & $\varangle$ & $I$ \\
\hline 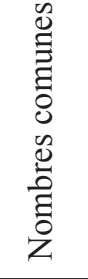 & & 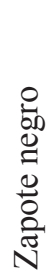 & & 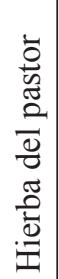 & 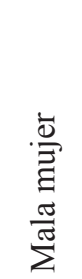 & 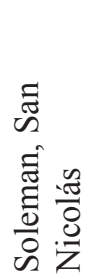 & 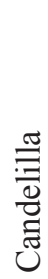 & 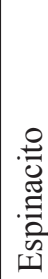 & 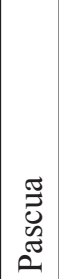 & 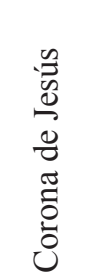 & 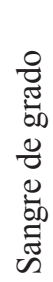 & 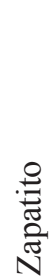 & $\begin{array}{l}\stackrel{0}{0} \\
\stackrel{0}{0}\end{array}$ & & $\frac{\vdots}{0}$ & 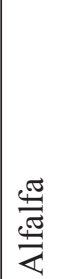 \\
\hline 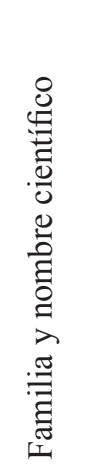 & 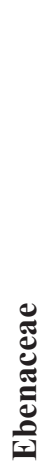 & 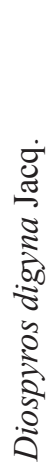 & 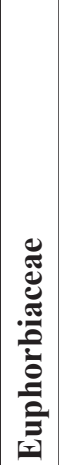 & 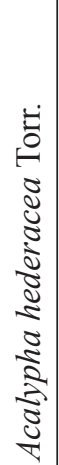 & 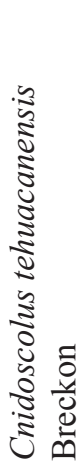 & 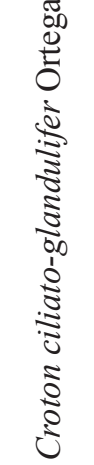 & 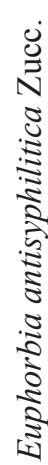 & $\begin{array}{l}0 \\
0 \\
0 \\
0 \\
0 \\
0 \\
0 \\
0 \\
0 \\
0 \\
0 \\
0 \\
0 \\
0 \\
0\end{array}$ & 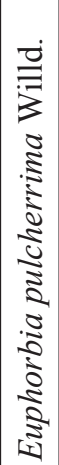 & 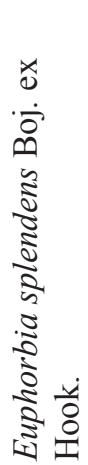 & $\begin{array}{l}0 \\
\infty \\
0 \\
0 \\
0 \\
0 \\
0 \\
0 \\
0 \\
0 \\
0 \\
0 \\
0 \\
0 \\
0 \\
0\end{array}$ & 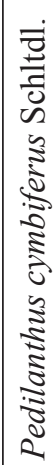 & 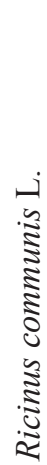 & 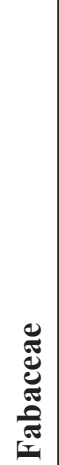 & 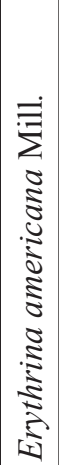 & 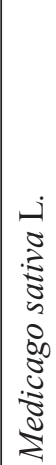 \\
\hline
\end{tabular}


Paredes-Flores et al.: Estudio etnobotánico de Zapotitlán Salinas, Puebla

\begin{tabular}{|c|c|c|c|c|c|c|c|c|c|c|c|c|c|c|c|c|c|c|c|}
\hline 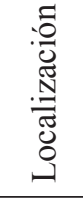 & $\begin{array}{l}\text { 至 } \\
\text { 导 }\end{array}$ & 寻 & & 己ี & & $\stackrel{\Xi}{\Xi}$ & & 荌 & & $\Xi$ & & $\stackrel{\Xi}{\Xi}$ & $\underset{\Xi}{\Xi}$ & 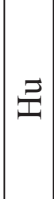 & 至 & $\Xi$ & $\stackrel{0}{\Sigma}$ & & 胥 \\
\hline $\begin{array}{l}\stackrel{\circ}{\mathbb{E}} \\
\stackrel{\Xi}{\Sigma}\end{array}$ & $\vec{U}$ & $\tilde{J}$ & & $\bar{\omega}$ & & $\tilde{J}$ & & $\bar{\sim}$ & & $\tilde{J}$ & & $\vec{U}$ & $\overrightarrow{\tilde{U}}$ & $\vec{U}$ & $\vec{U}$ & $\vec{U}$ & $\dot{\sim}$ & & تَ \\
\hline 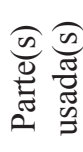 & 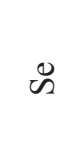 & 它 & & $\stackrel{\pi}{\sigma}$ & & $\begin{array}{l}I \\
\hat{2} \\
\hat{F}\end{array}$ & & $I$ & & 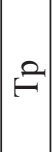 & & $I$ & $\begin{array}{l}\stackrel{\pi}{ت} \\
\tilde{I}\end{array}$ & $I$ & 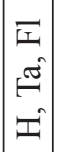 & 厌 & $\begin{array}{l}\tilde{\sigma} \\
\mathcal{I}^{2}\end{array}$ & & $\begin{array}{l}\sum^{\pi} \\
\vec{I} \\
\vec{I}\end{array}$ \\
\hline $\begin{array}{l}\infty \\
0 \\
\mathscr{D}\end{array}$ & $\stackrel{\sim}{\sim}$ & $\mathrm{N}$ & & $m$ & & $\begin{array}{l}\nabla \\
0\end{array}$ & & $\nabla$ & & 0 & & 6 & $\nabla$ & $\frac{7}{8}$ & $\begin{array}{l}0 \\
t\end{array} \mid$ & $\begin{array}{l}0 \\
f^{2}\end{array}$ & $\nabla$ & & $\begin{array}{l}\text { ב } \\
\text { व }\end{array}$ \\
\hline $\begin{array}{l}0 \\
\text { D } \\
\dot{0} \\
\dot{0}\end{array}$ & $\begin{array}{l}\stackrel{0}{+} \\
\text { in } \\
\stackrel{n}{d}\end{array}$ & 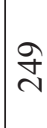 & & $\vec{m}$ & & 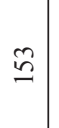 & & $\stackrel{\infty}{\curvearrowright}$ & & $\stackrel{\circ}{2}$ & & 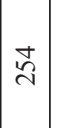 & $\stackrel{ \pm}{\beth}$ & $\vec{n}$ & $\stackrel{\infty}{n}$ & กี & ڤิ & & 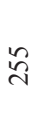 \\
\hline 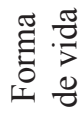 & $I$ & $I$ & & 宗 & & $\Psi$ & & $I$ & & $I$ & & $I$ & $I$ & $I$ & $I$ & $I$ & $I$ & & $\varangle$ \\
\hline 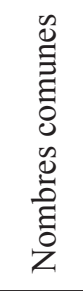 & 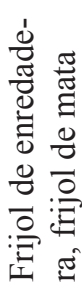 & 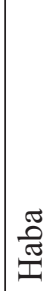 & & 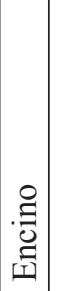 & & 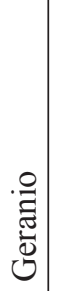 & & 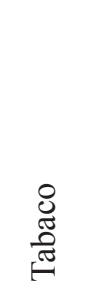 & & $\stackrel{\circ}{\stackrel{ }{\Xi}}$ & & 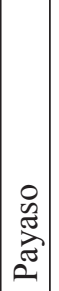 & 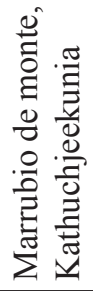 & 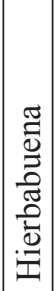 & 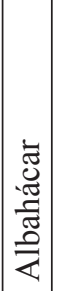 & : & 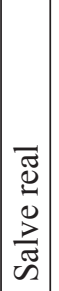 & & 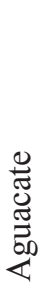 \\
\hline 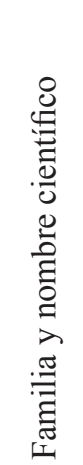 & 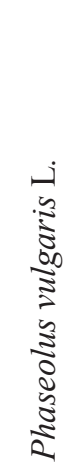 & 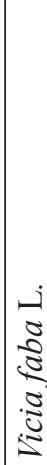 & 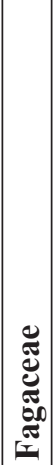 & 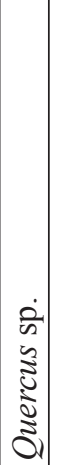 & 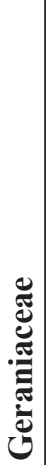 & 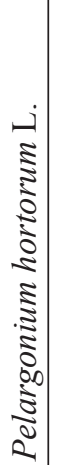 & |. & 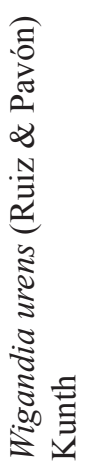 & 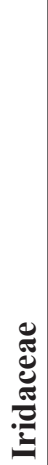 & 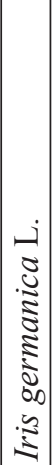 & 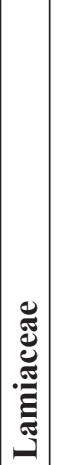 & 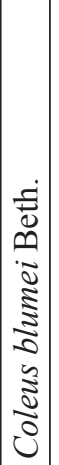 & 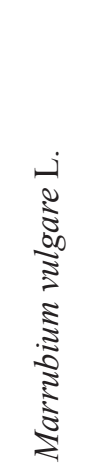 & 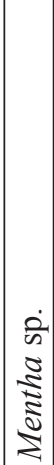 & 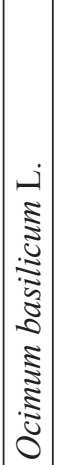 & $\begin{array}{l} \\
\dot{2} \\
\frac{2}{2} \\
\frac{2}{2} \\
\dot{0}\end{array}$ & $\begin{array}{c} \\
0 \\
2 \\
0 \\
: \\
\frac{2}{2} \\
5 \\
5\end{array}$ & 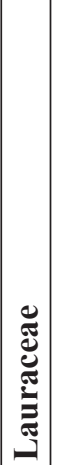 & 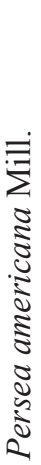 \\
\hline
\end{tabular}




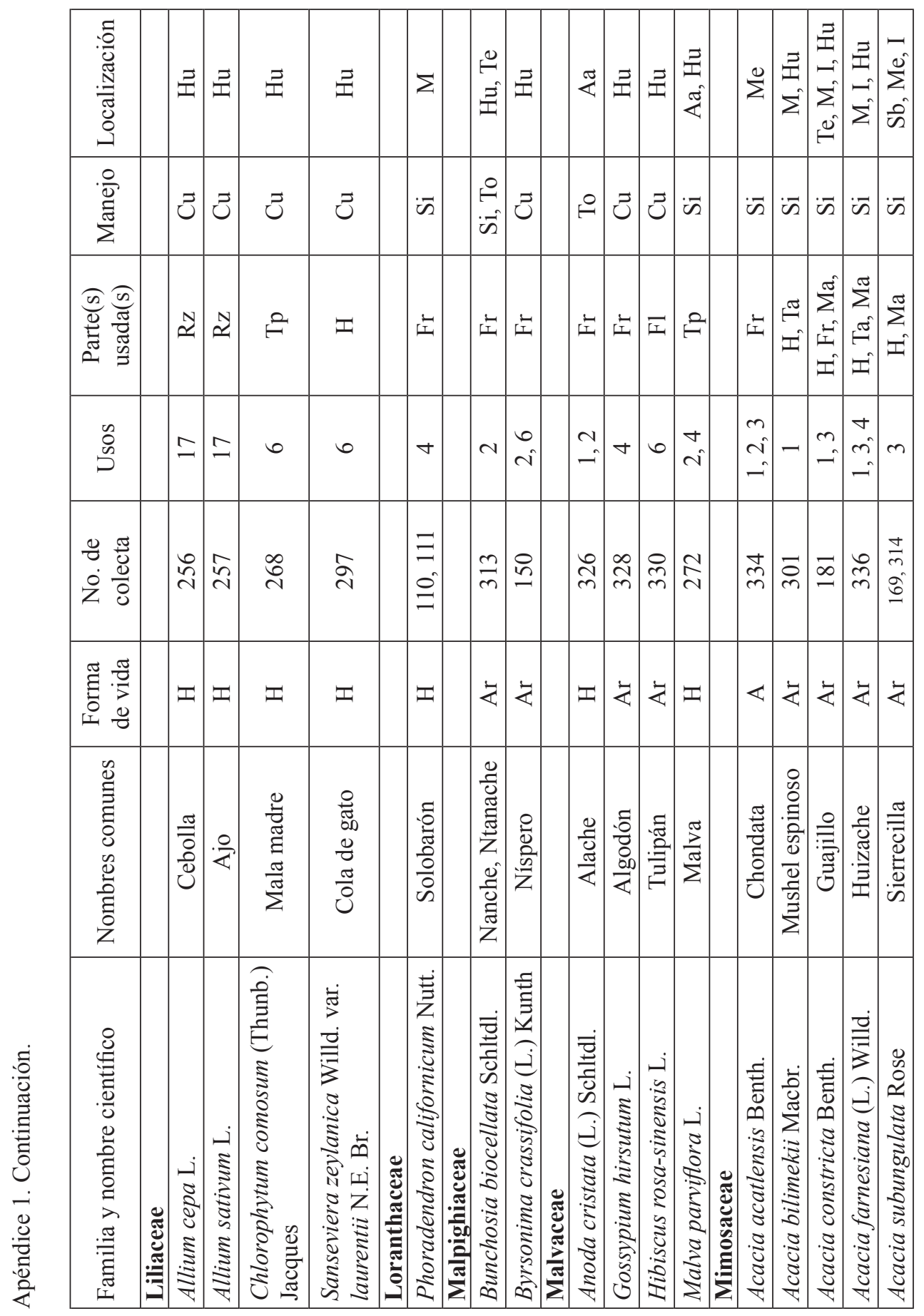


Paredes-Flores et al.: Estudio etnobotánico de Zapotitlán Salinas, Puebla

\begin{tabular}{|c|c|c|c|c|c|c|c|c|c|c|c|c|c|c|c|}
\hline 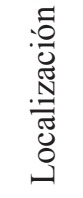 & $\Xi$ & 至 & ஜึ & $\sum_{i}^{e}$ & 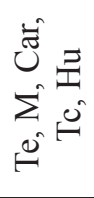 & $\stackrel{\oplus}{\tilde{E}}$ & & $\Xi$ & 忢 & $\vec{\Xi}$ & 焉 & & $\underset{\Xi}{\Xi}$ & & 茎 \\
\hline$\frac{\stackrel{\circ}{\mathscr{E}}}{\sum_{\Sigma}^{ \pm}}$ & $\vec{~}$ & $\vec{U}$ & $\bar{\sim}$ & $\ddot{\sim}$ & $\begin{array}{l}\stackrel{0}{1} \\
i \tilde{n}\end{array}$ & $\bar{\sim}$ & & $\vec{~}$ & $\vec{U}$ & $\vec{U}$ & $\vec{~}$ & & $\Xi$ & & ت \\
\hline 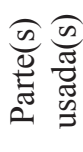 & $\begin{array}{l}\text { 宅 } \\
\stackrel{\tilde{E}}{E} \\
\tilde{I}\end{array}$ & 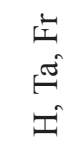 & $\stackrel{\pi}{0}$ & $\begin{array}{l}\stackrel{\pi}{I} \\
\tilde{I}\end{array}$ & 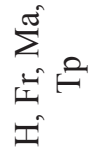 & $\bar{I}$ & & 它 & 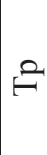 & 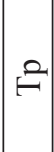 & 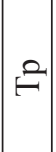 & & 空 & & 空 \\
\hline $\begin{array}{l}n \\
0 \\
\infty\end{array}$ & $\begin{array}{l}\text { ri } \\
\therefore i n \\
\therefore i\end{array}$ & $\stackrel{n}{\stackrel{n}{i}}$ & $\begin{array}{l}\nabla \\
m\end{array}$ & $\stackrel{n}{\sim}$ & $\stackrel{n}{n}$ & 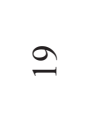 & & $\sim$ & $\frac{\Xi}{\sigma}$ & $\begin{array}{l} \pm \\
0\end{array}$ & $\frac{\nabla}{0}$ & & $\sim$ & & $\begin{array}{l}+ \\
\text { d }\end{array}$ \\
\hline $\begin{array}{l}0 \\
0 \\
\dot{0} \\
\dot{0}\end{array}$ & $\stackrel{8}{ }$ & $\hat{m}$ & $\overrightarrow{\widetilde{N}}$ & 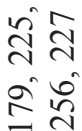 & $\stackrel{\circ}{\circ}$ & $\underline{b}$ & & $\frac{\Xi}{\nabla}$ & $\frac{n}{\gamma}$ & $\begin{array}{l}0 \\
\gamma\end{array}$ & $\frac{\gamma}{\gamma}$ & & $\stackrel{\sim}{\underset{工}{\sim}}$ & & $\frac{\infty}{\gamma}$ \\
\hline 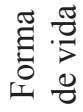 & $\varangle$ & $\varangle$ & $\varangle$ & 文 & 这 & 完 & & 文 & $\varangle$ & $\varangle$ & $\ll$ & & $I$ & & 这 \\
\hline 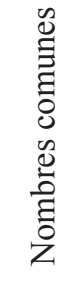 & 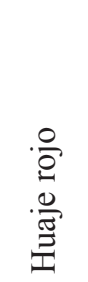 & 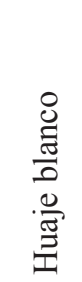 & 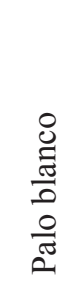 & 已ُ & 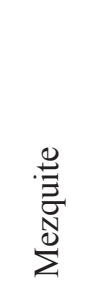 & 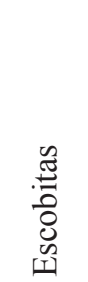 & & .0 & $\frac{8}{0}$ & 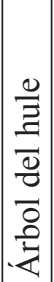 & 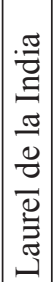 & & 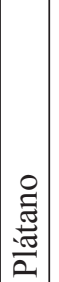 & & 疍 \\
\hline 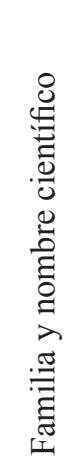 & 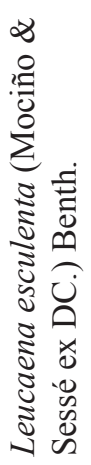 & 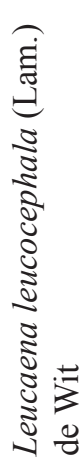 & 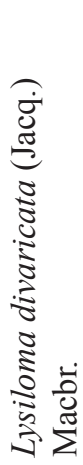 & 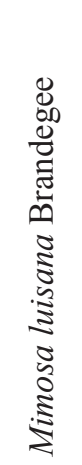 & 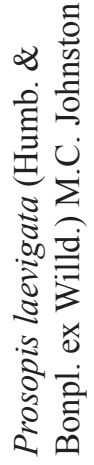 & 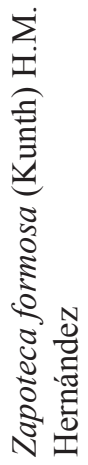 & 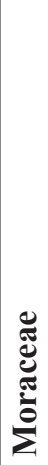 & 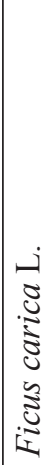 & 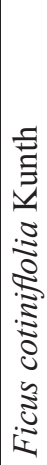 & 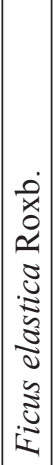 & 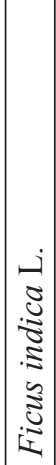 & 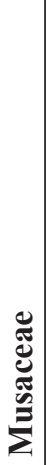 & 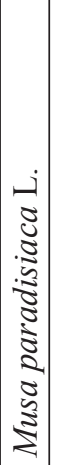 & 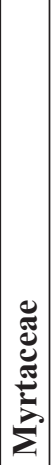 & 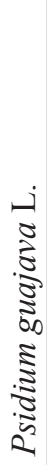 \\
\hline
\end{tabular}


Acta Botanica Mexicana 79: 13-61 (2007)

\begin{tabular}{|c|c|c|c|c|c|c|c|c|c|c|c|c|c|c|c|c|}
\hline 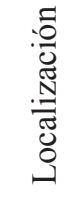 & & $\begin{array}{l}\overrightarrow{\mid \vec{y}} \\
\stackrel{\oplus}{\oplus}\end{array}$ & - & & 竎 & 寻 & & $\underset{\Xi}{\Xi}$ & & $\begin{array}{l}\vec{\Xi} \\
\tilde{\Xi}\end{array}$ & & 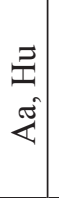 & & $\underset{\psi}{\mathbb{2}}$ & & $\Sigma$ \\
\hline 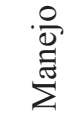 & & $\begin{array}{l}\vec{U} \\
\dot{\omega}\end{array}$ & $\bar{\sim}$ & & $\vec{z}$ & $\vec{~}$ & & $\vec{U}$ & & $\begin{array}{l}\dot{H} \\
\dot{\omega}\end{array}$ & & $\bar{\omega}$ & & $\begin{array}{l}0 \\
0 \\
0\end{array}$ & & $\bar{\sim}$ \\
\hline 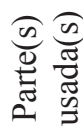 & & $\stackrel{2}{E}$ & $\begin{array}{l}\vec{I} \\
\stackrel{\tilde{I}}{=} \\
\tilde{I}\end{array}$ & & $\mid \begin{array}{l}\hat{f} \\
\vec{I} \\
\vec{I}\end{array}$ & $\vec{E}$ & & $\Leftrightarrow$ & & $\begin{array}{l}\bar{I} \\
\bar{I}\end{array}$ & & $\stackrel{2}{E}$ & & 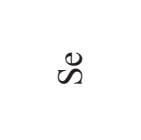 & & e \\
\hline $\begin{array}{l}\mathscr{D} \\
\mathscr{D} \\
\emptyset\end{array}$ & & 0 & n & & $\begin{array}{l}0 \\
+\end{array}$ & $\begin{array}{l}0 \\
+\end{array}$ & & 6 & & 6 & & $\nabla$ & & $\sim$ & & $\nabla$ \\
\hline $\begin{array}{l}\frac{\pi}{0} \\
\dot{0} \\
\dot{0} \\
\end{array}$ & & రి & $\frac{\curvearrowright}{\gamma}$ & & 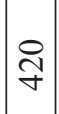 & $\stackrel{n}{\Xi}$ & & $\bar{n}$ & & $\overrightarrow{\mathcal{\gamma}}$ & & $\stackrel{\infty}{\sim}$ & & よ & & $\stackrel{\Im}{\vartheta}$ \\
\hline 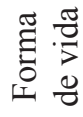 & & $\varangle$ & $I$ & & $I$ & 文 & & $\dot{z}$ & & $I$ & & $I$ & & $I$ & & $I$ \\
\hline 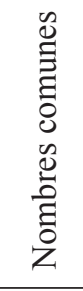 & & 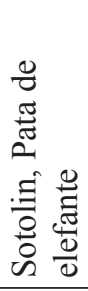 & 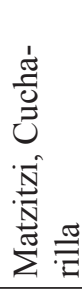 & & 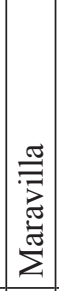 & 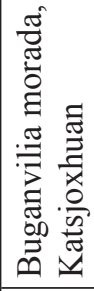 & & 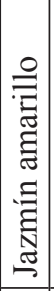 & & 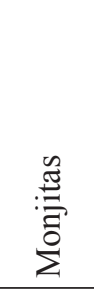 & & 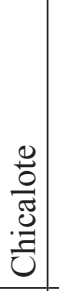 & & 量 & & 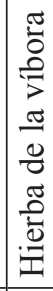 \\
\hline 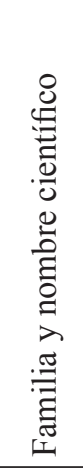 & 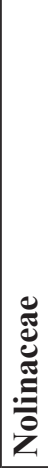 & 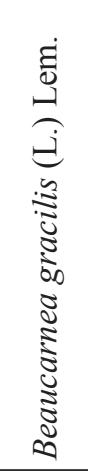 & 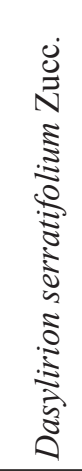 & 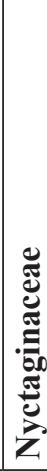 & 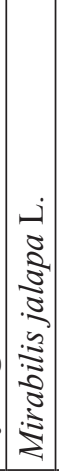 & 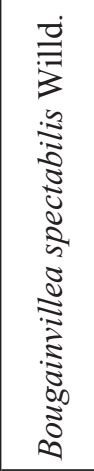 & 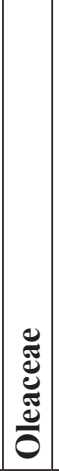 & 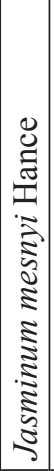 & 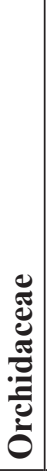 & 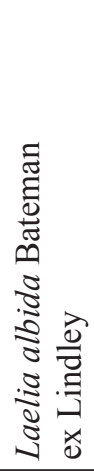 & 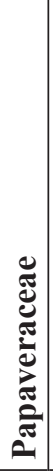 & 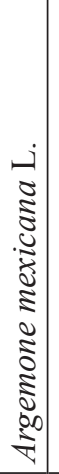 & 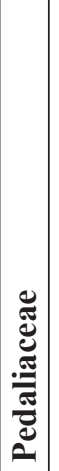 & 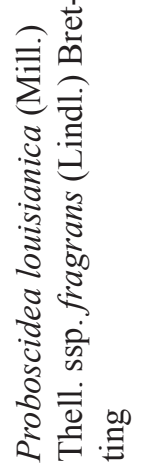 & 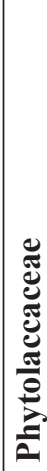 & 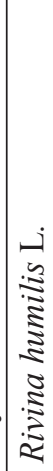 \\
\hline
\end{tabular}


Paredes-Flores et al.: Estudio etnobotánico de Zapotitlán Salinas, Puebla

\begin{tabular}{|c|c|c|c|c|c|c|c|c|c|c|c|c|c|c|c|c|c|}
\hline 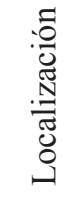 & & $\stackrel{\Xi}{\Xi}$ & & 吾 & & $\stackrel{0}{\Sigma}$ & 要 & $\stackrel{\pi}{\gtrless}$ & 变 & 茎 & $\sum_{\stackrel{0}{\oplus}}$ & $\underset{\psi}{\pi}$ & $\sum_{\Sigma}^{0}$ & 孚 & 党 & 胥 & $\underset{\pi}{\pi}$ \\
\hline $\begin{array}{l}\stackrel{\circ}{\stackrel{\Xi}{\Xi}} \\
\sum\end{array}$ & & $\vec{U}$ & & $\vec{~}$ & & $\bar{\sim}$ & $\bar{\omega}$ & $\bar{n}$ & $\overline{i s}$ & $\stackrel{0}{1}$ & $\bar{\sim}$ & $\vec{U}$ & $\bar{\sim}$ & is & $\bar{\sim}$ & $\bar{\omega}$ & $\bar{\omega}$ \\
\hline 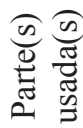 & & $I$ & & $\underline{n}$ & & $I$ & $I$ & $I$ & $I$ & 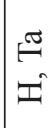 & $I$ & $I$ & $I$ & $I$ & $\Psi$ & $\Psi$ & $I$ \\
\hline $\begin{array}{l}n \\
0 \\
\infty \\
\wp\end{array}$ & & $\begin{array}{l}0 \\
\text { i }\end{array}$ & & 6 & & - & -1 & - & - & $\stackrel{\nabla}{-}$ & - & - & - & - & - & - & - \\
\hline $\begin{array}{l}0 \\
\text { D } \\
\dot{0} \\
\dot{0}\end{array}$ & & $\begin{array}{l}\exists \\
\Xi \\
\stackrel{\ominus}{\Xi}\end{array}$ & & $\begin{array}{l}\infty \\
\infty \\
\sim \\
\tilde{n}\end{array}$ & & 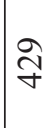 & $\begin{array}{c}\stackrel{0}{\sim} \\
\stackrel{m}{\forall}\end{array}$ & $\overrightarrow{\tilde{F}}$ & $\underset{\sim}{\mathscr{\gamma}}$ & $\ddot{\tilde{\sigma}}$ & $\stackrel{+}{+}$ & $\stackrel{n}{\dddot{m}}$ & 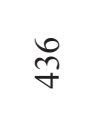 & $\stackrel{\tilde{m}}{+}$ & $\stackrel{\infty}{\stackrel{\infty}{\forall}}$ & $\underset{\sim}{\stackrel{ \pm}{N}}$ & $\frac{r}{n}$ \\
\hline 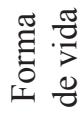 & & $I$ & & 完 & & $I$ & $\Psi$ & $I$ & $I$ & $I$ & $I$ & $I$ & $I$ & $I$ & $I$ & $I$ & $I$ \\
\hline 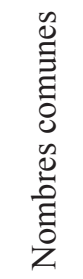 & & 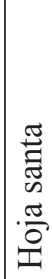 & & 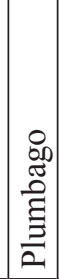 & & 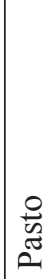 & $\mid \begin{array}{c}0 \\
\frac{0}{0} \\
\tilde{U} \\
0\end{array}$ & 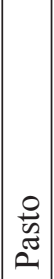 & $\begin{array}{c}0 \\
\text { 营 } \\
\text { 20 }\end{array}$ & Ů & 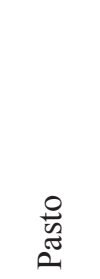 & $\begin{array}{l}0 \\
0 \\
\text { a } \\
2 \\
2\end{array}$ & 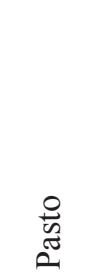 & 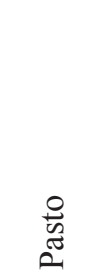 & 营 & 兽 & 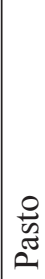 \\
\hline 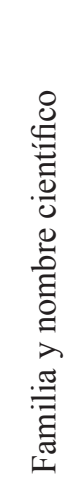 & 苞 & 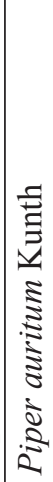 & 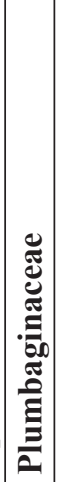 & 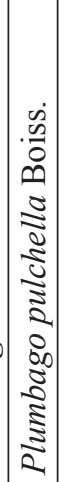 & 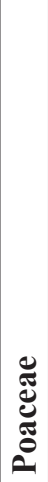 & 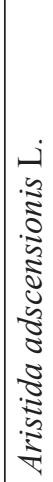 & 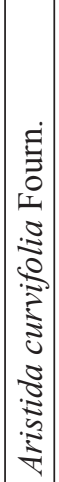 & 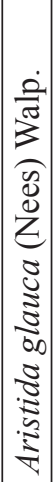 & 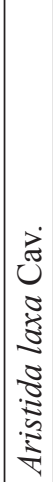 & 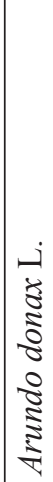 & 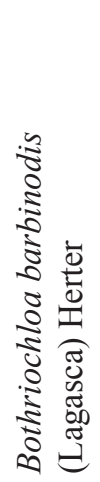 & 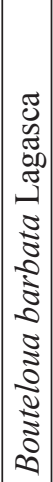 & 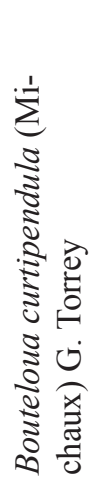 & 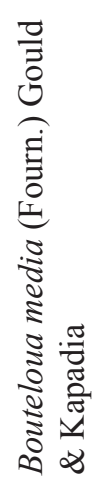 & 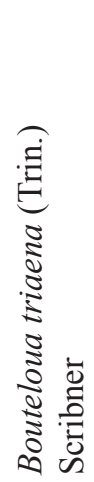 & 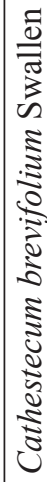 & 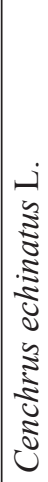 \\
\hline
\end{tabular}


Acta Botanica Mexicana 79: 13-61 (2007)

\begin{tabular}{|c|c|c|c|c|c|c|c|c|c|c|c|c|c|c|c|}
\hline 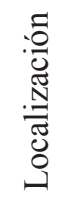 & $\Sigma$ & 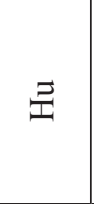 & $\stackrel{\pi}{4}$ & 胥 & $\stackrel{\pi}{4}$ & $\Sigma$ & $\Sigma$ & $\Sigma$ & $\Sigma$ & $\Xi$ & $\Sigma$ & $\sum$ & $\Sigma$ & $\Sigma$ & $\Sigma$ \\
\hline$\frac{.}{\stackrel{0}{\Xi}}$ & $\bar{n}$ & $\vec{U}$ & $\bar{\omega}$ & $\ddot{\omega}$ & $\bar{\omega}$ & $\bar{\omega}$ & $\bar{\omega}$ & $\bar{\omega}$ & $\bar{\omega}$ & $\bar{\omega}$ & $\bar{n}$ & $\bar{\sim}$ & $\bar{\omega}$ & $\bar{\sim}$ & $\bar{\sim}$ \\
\hline 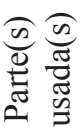 & $I$ & $I$ & $I$ & $I$ & $I$ & $I$ & $I$ & $I$ & $I$ & $I$ & $I$ & $I$ & $I$ & $I$ & $I$ \\
\hline $\begin{array}{l}\mathscr{n} \\
0 \\
\infty \\
\infty\end{array}$ & - & $\begin{array}{l}0 \\
\nabla^{\prime}\end{array}$ & - & - & - & - & - & - & - & - & - & - & -1 & - & - \\
\hline 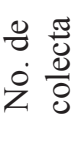 & $\frac{\infty}{m}$ & શે & 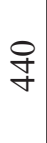 & 尹 & 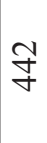 & $\begin{array}{l}\stackrel{\circ}{N} \\
\stackrel{n}{N} \\
\frac{n}{n}\end{array}$ & $\begin{array}{l}\tilde{N} \\
\tilde{n} \\
\tilde{N} \\
\text { ñ }\end{array}$ & $\stackrel{\Re}{\&}$ & $\stackrel{\circ}{m}$ & $\underset{\forall}{\&}$ & $\stackrel{n}{f}$ & $\begin{array}{l}0 \\
\dot{y}\end{array}$ & $\underset{f}{f}$ & $\stackrel{\infty}{+}$ & $\stackrel{g}{g}$ \\
\hline 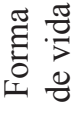 & $I$ & $I$ & $I$ & $I$ & $I$ & $I$ & $I$ & $I$ & $I$ & $I$ & $I$ & $I$ & $I$ & $\Psi$ & $I$ \\
\hline 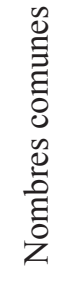 & 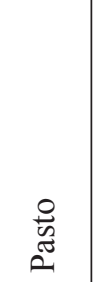 & 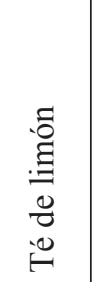 & \begin{tabular}{c}
0 \\
\multirow{2}{*}{} \\
$\stackrel{\mathbb{Z}}{0 .}$
\end{tabular} & 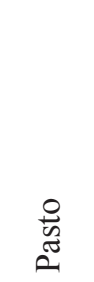 & 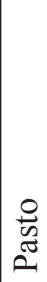 & 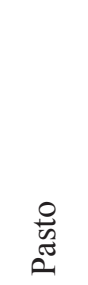 & 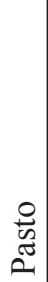 & 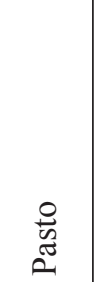 & 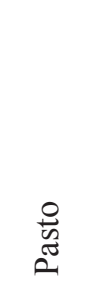 & 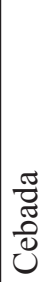 & 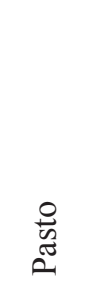 & 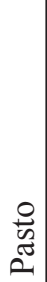 & $\mid \begin{array}{c}0 \\
0 \\
0 \\
0 \\
0\end{array}$ & 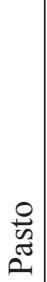 & 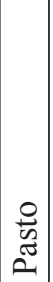 \\
\hline 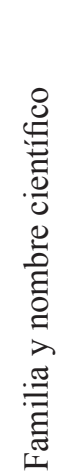 & 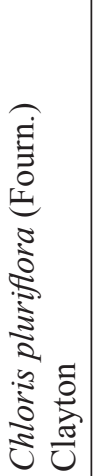 & 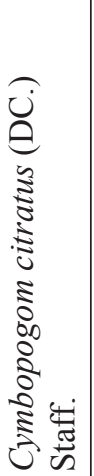 & 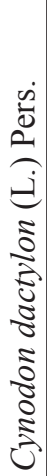 & 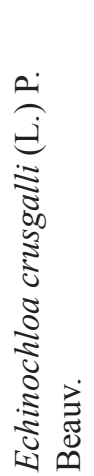 & 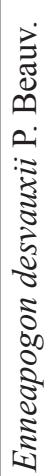 & 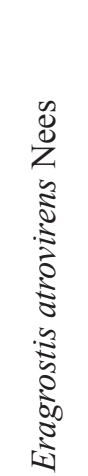 & 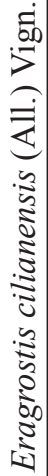 & 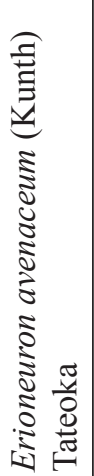 & 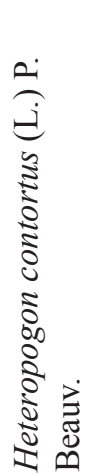 & 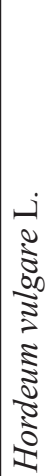 & 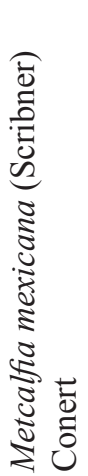 & 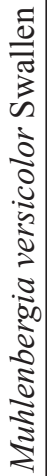 & 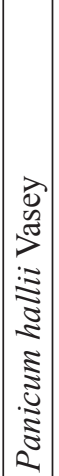 & 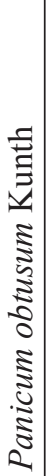 & 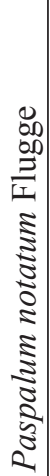 \\
\hline
\end{tabular}


Paredes-Flores et al.: Estudio etnobotánico de Zapotitlán Salinas, Puebla

\begin{tabular}{|c|c|c|c|c|c|c|c|c|c|c|c|c|c|}
\hline 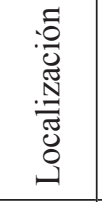 & $\Sigma$ & $\Sigma$ & $\Sigma$ & $\Sigma$ & 寻 & $\Sigma$ & 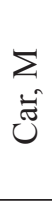 & $\mid \begin{array}{l}\vec{y} \\
\stackrel{\vec{\psi}}{\alpha}\end{array}$ & $\sum_{\oplus}^{0}$ & 寻 & $\begin{array}{l}\Xi \\
\Xi \\
\text { 离 }\end{array}$ & 可 & 现 \\
\hline 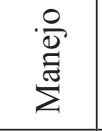 & $\bar{n}$ & $\bar{n}$ & $\bar{n}$ & $\bar{n}$ & $\vec{J}$ & $\bar{\omega}$ & $\bar{n}$ & $\vec{\Xi}$ & $\bar{n}$ & $\vec{U}$ & $\bar{\omega}$ & $\vec{U}$ & $\vec{\Xi}$ \\
\hline 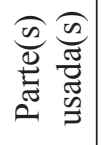 & $\Psi$ & $\Psi$ & $\Psi$ & $\Psi$ & 空 & $\Psi$ & I & $\begin{array}{l}0 \\
\tilde{n} \\
\tilde{\Theta} \\
-1\end{array}$ & $\Leftrightarrow$ & 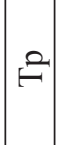 & $\begin{array}{l}\tilde{F} \\
\tilde{I}\end{array}$ & $I$ & $\mathbf{I}$ \\
\hline $\begin{array}{l}\text { b } \\
\text { b } \\
0\end{array}$ & - & - & - & - & - & - & - & $\stackrel{N}{-}$ & $\nabla$ & 0 & & 6 & 6 \\
\hline $\begin{array}{ll}0 & \pi \\
0 \\
\dot{z} & \frac{0}{0} \\
\dot{\delta}\end{array}$ & $\stackrel{\circ}{r}$ & ᄅे & $\bar{n}$ & $\stackrel{\approx}{\approx}$ & 吕 & ṽ & $\stackrel{n}{q}$ & 苞 & 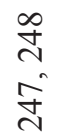 & 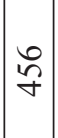 & 啻 & $\stackrel{\infty}{\sim}$ & $\hat{n}$ \\
\hline
\end{tabular}

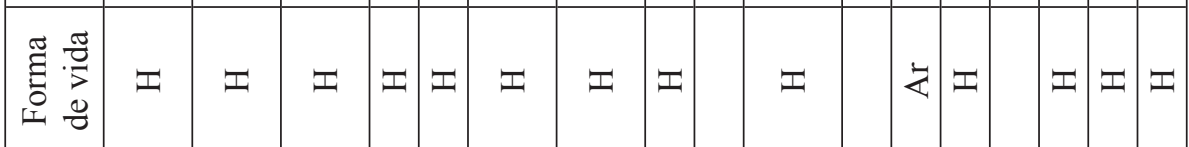

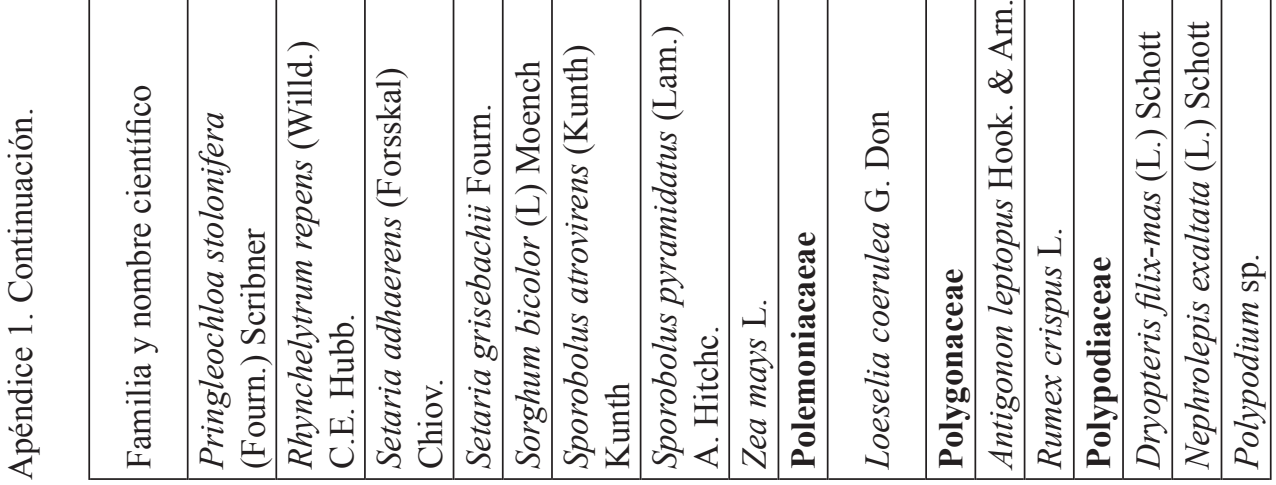




\begin{tabular}{|c|c|c|c|c|c|c|c|c|c|c|c|c|c|c|c|c|c|c|}
\hline 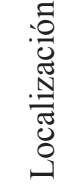 & & $\underset{\Xi}{\Xi}$ & $\begin{array}{l}\vec{\Xi} \\
\text { 芦 }\end{array}$ & $\Sigma$ & & $\stackrel{\Xi}{\Xi}$ & & $\underset{\Xi}{\Xi}$ & & $\vec{E}$ & $\oplus$ & & $\tilde{U}$ & $\underset{\Xi}{\Xi}$ & $\Xi$ & 竎 & & 吾 \\
\hline $\begin{array}{l}\frac{0}{\mathbb{E}} \\
\stackrel{\Xi}{\Xi}\end{array}$ & & $\vec{U}$ & $\ominus$ & $\bar{s}$ & & $\mid \begin{array}{l}0 \\
\hat{\omega} \\
\hat{s}\end{array}$ & & $\vec{U}$ & & $\ominus$ & $\ddot{\omega}$ & & $\bar{s}$ & $\vec{U}$ & $\vec{U}$ & $\vec{U}$ & & ت \\
\hline 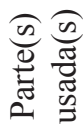 & & $\begin{array}{l}\vec{I} \\
\pm \\
I\end{array}$ & 牙 & $I$ & & 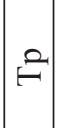 & & 它 & & $\stackrel{2}{F}$ & 它 & & $\Psi$ & 它 & 它 & $\bar{I}$ & & $\stackrel{\overrightarrow{1}}{\overrightarrow{1}}$ \\
\hline $\begin{array}{l}\mathscr{n} \\
\stackrel{\infty}{p} \\
\mathscr{p}\end{array}$ & & 6 & $\stackrel{+}{\sim}$ & $\stackrel{\sim}{\curvearrowleft}$ & & $\nabla$ & & $\begin{array}{l}0 \\
\text { i }\end{array}$ & & 0 & $\nabla$ & & - & $N$ & $\sim$ & $\begin{array}{l}0 \\
\nabla^{2}\end{array}$ & & r \\
\hline $\begin{array}{l}0 \\
0 \\
\dot{0} \\
\dot{0}\end{array}$ & & 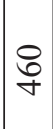 & $\begin{array}{l}0 \\
\text { ปे } \\
\text { ᄋे } \\
\text { ปे }\end{array}$ & $\vec{\sim}$ & & $\hat{n}$ & & $\stackrel{\infty}{ \pm}$ & & $\bar{\sigma}$ & $\begin{array}{l}\stackrel{n}{0} \\
\substack{0 \\
0}\end{array}$ & & $\frac{0}{m}$ & ర్ & $\begin{array}{l}\hat{\delta} \\
+\end{array}$ & ठ্ণ & & શิ \\
\hline 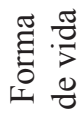 & & $I$ & $I$ & $I$ & & $I$ & & 交 & & 安 & 完 & & 文 & $\varangle$ & $\varangle$ & 安 & & $\ll$ \\
\hline 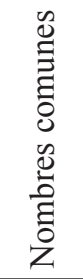 & & 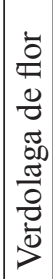 & 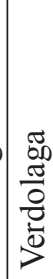 & 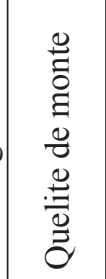 & & 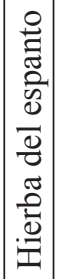 & & 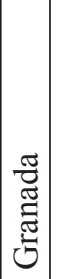 & & $\frac{.0}{.07}$ & 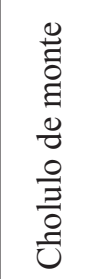 & & & 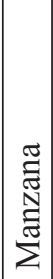 & 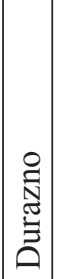 & 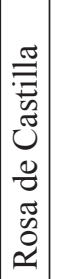 & & 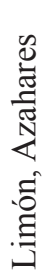 \\
\hline 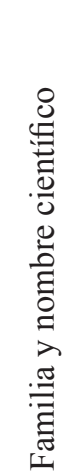 & 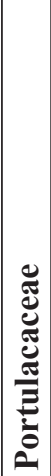 & 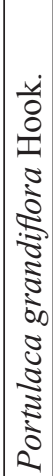 & \begin{tabular}{|l}
0 \\
0 \\
0 \\
0 \\
0 \\
0 \\
0 \\
0 \\
0 \\
0 \\
0 \\
0 \\
3 \\
0 \\
0 \\
0 \\
0
\end{tabular} & 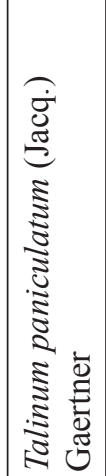 & 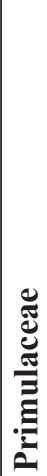 & 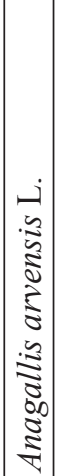 & 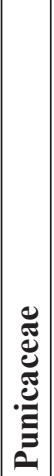 & 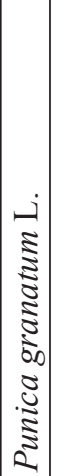 & 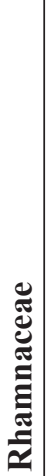 & 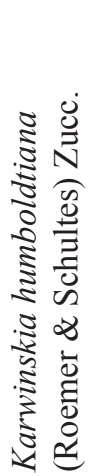 & 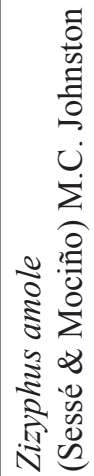 & 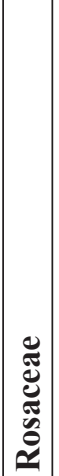 & 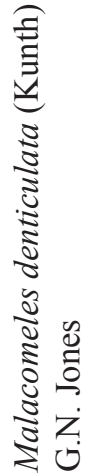 & $\begin{array}{c}\dot{2} \\
\frac{2}{2} \\
\frac{3}{3} \\
\frac{3}{z}\end{array}$ & $\left|\begin{array}{c}0 \\
0 \\
0 \\
0 \\
0 \\
0 \\
0 \\
\vdots \\
\vdots \\
\vdots \\
\vdots \\
\vdots \\
0\end{array}\right|$ & 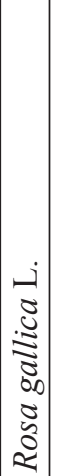 & 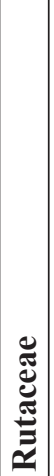 & 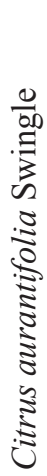 \\
\hline
\end{tabular}


Paredes-Flores et al.: Estudio etnobotánico de Zapotitlán Salinas, Puebla

\begin{tabular}{|c|c|c|c|c|c|c|c|c|c|c|c|c|c|c|c|c|c|c|}
\hline 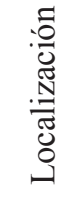 & 至 & $\Xi$ & $\Xi$ & 夛 & 寻 & 寻 & & $\Xi$ & $\Xi$ & & 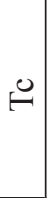 & & 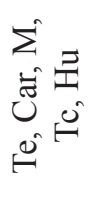 & & $\begin{array}{l}\sum^{0} \\
\Xi\end{array}$ & 忢 & $\stackrel{\mathbb{Z}}{4}$ & 学 \\
\hline$\frac{. ㅇ}{\stackrel{\Xi}{\Xi}}$ & $\vec{U}$ & $\vec{U}$ & $\vec{U}$ & $\vec{J}$ & $\vec{U}$ & $\vec{U}$ & & 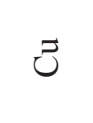 & $\vec{J}$ & & $\bar{\sim}$ & & $\bar{\sim}$ & & $\overrightarrow{\tilde{s}}$ & $\vec{U}$ & $\bar{\omega}$ & $\bar{\omega}$ \\
\hline 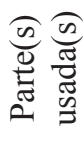 & 它 & 它 & 它 & 空 & 空 & $\stackrel{N}{2}$ & & $\stackrel{0}{H}$ & 离 & & $I$ & & 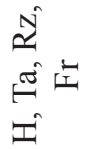 & & 㝎 & 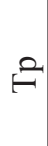 & 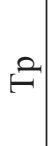 & $\stackrel{2}{E}$ \\
\hline $\begin{array}{l}\infty \\
0 \\
\infty \\
b\end{array}$ & $\sim$ & $\sim \mid$ & $\sim \mid$ & $\sim$ & $\sim$ & $\left|\begin{array}{l}0 \\
f\end{array}\right|$ & & $\sim$ & $\begin{array}{l} \pm \\
\text { v }\end{array}$ & & $\nabla$ & & in & & 드 & 0 & $\nabla$ & $\nabla$ \\
\hline $\begin{array}{l}\text { D } \\
\dot{0} \\
\dot{0} \\
\dot{0}\end{array}$ & $\begin{array}{l}n \\
\sigma \\
\dot{f}\end{array}$ & 잉 & $\hat{\sigma}$ & $\underset{⿱}{\infty}$ & જे & 守 & & $\vec{F}$ & $\hat{n}$ & & $\hat{n}$ & & 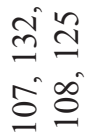 & & $\frac{\curvearrowright}{\sim}$ & $\stackrel{ }{\stackrel{\gamma}{\sigma}}$ & $\stackrel{ }{ }$ & $\begin{array}{l}\infty \\
0 \\
m \\
m \\
n\end{array}$ \\
\hline 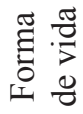 & $\varangle$ & $\varangle$ & $\ll$ & $\varangle$ & $\varangle$ & 文 & & $\varangle$ & $\varangle$ & & $I$ & & 完 & & 宗 & 宗 & $I$ & $I$ \\
\hline 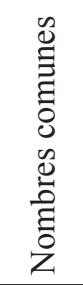 & 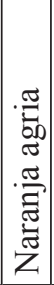 & : & 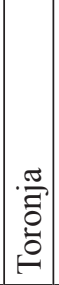 & 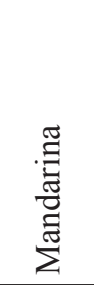 & 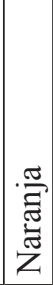 & 胥 & & 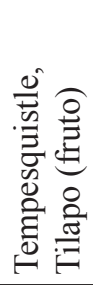 & 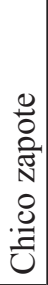 & & 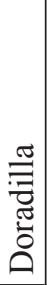 & & $\begin{array}{l}\stackrel{0}{\Xi} \\
\stackrel{0}{0} \\
\stackrel{0}{>}\end{array}$ & & 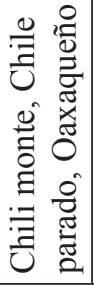 & 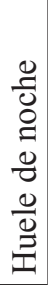 & $\begin{array}{c} \\
\frac{\pi}{\vec{E}} \\
\frac{\vec{a}}{F}\end{array}$ & 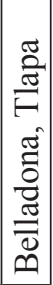 \\
\hline 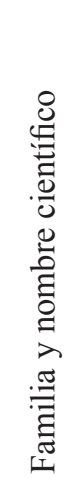 & 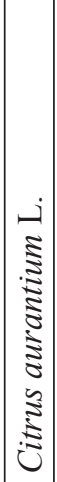 & 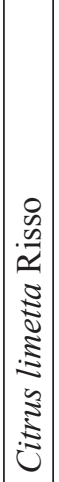 & 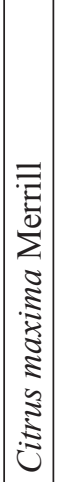 & 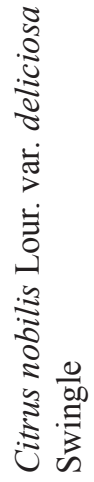 & 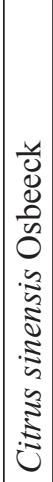 & 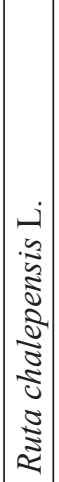 & 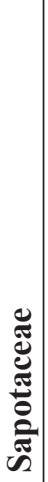 & 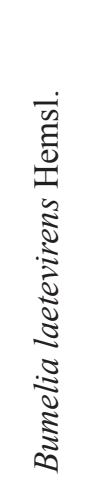 & 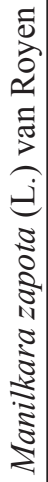 & 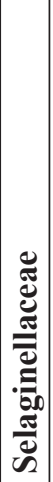 & 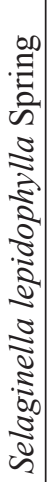 & 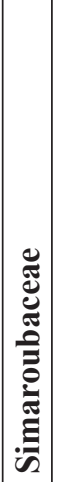 & 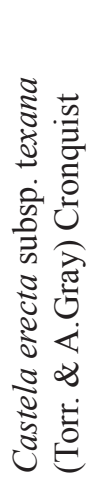 & 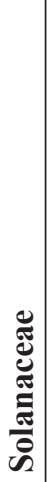 & 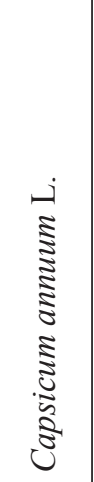 & 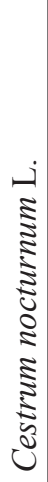 & 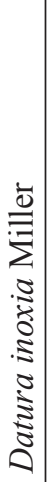 & 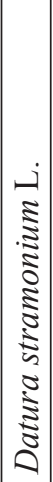 \\
\hline
\end{tabular}




\begin{tabular}{|c|c|c|c|c|c|c|c|c|c|c|c|c|c|c|c|}
\hline 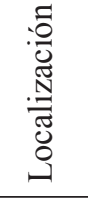 & 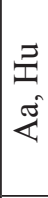 & ஜ & $\stackrel{\pi}{4}$ & 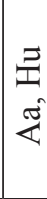 & ت્1 & $\Xi$ & 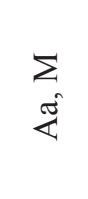 & $\begin{array}{l}\sum \\
\tilde{\pi}^{\tilde{\psi}}\end{array}$ & & $\sum^{0}$ & & $\begin{array}{l}\vec{\Xi} \\
\Sigma\end{array}$ & & $\underbrace{\tilde{E}}$ & $\begin{array}{l}\vec{I} \\
\stackrel{\tilde{U}}{\tilde{E}} \\
\ddot{\tilde{U}}\end{array}$ \\
\hline 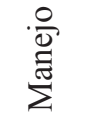 & $\vec{~}$ & $\bar{\sim}$ & $\bar{\sim}$ & $\vec{~}$ & $\vec{U}$ & $\bar{\sim}$ & $\ddot{\sim}$ & $\bar{\sim}$ & & $\ddot{\sim}$ & & $\ddot{\sim}$ & & $\bar{\sim}$ & $\ddot{\sim}$ \\
\hline 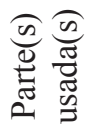 & 空 & $\stackrel{2}{1}$ & $I$ & 它 & $\begin{array}{l}\vec{I} \\
\stackrel{\tilde{E}}{F}\end{array}$ & 袁 & $\vec{I}$ & $\bar{I}$ & & 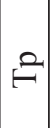 & & 空 & & $\underline{E}$ & $\begin{array}{l}\dot{1} \\
\dot{I}\end{array}$ \\
\hline $\begin{array}{l}\infty \\
0 \\
\emptyset\end{array}$ & $\sim$ & $\nabla$ & $\nabla$ & $\begin{array}{l}\nabla \\
\text { vi }\end{array}$ & 6 & v & $\nabla$ & $\nabla$ & & $\nabla$ & & $\begin{array}{l}\nabla \\
m\end{array}$ & & $\nabla$ & $\begin{array}{l}\nabla \\
\text { i }\end{array}$ \\
\hline $\begin{array}{ll}0 & \frac{\pi}{0} \\
\dot{0} & \frac{0}{0} \\
Z & 0\end{array}$ & $\stackrel{\Re}{\stackrel{\Upsilon}{\gamma}}$ & $\stackrel{\curvearrowright}{\Delta}$ & 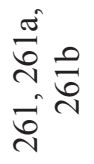 & $\stackrel{9}{=}$ & $\underset{\forall}{\stackrel{大}{\forall}}$ & ○ & 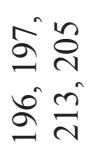 & 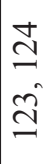 & & 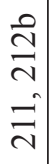 & & 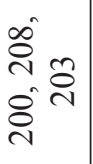 & & $\frac{a}{\sqrt{N}}$ & 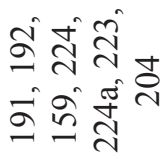 \\
\hline 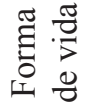 & $I$ & $I$ & 文 & $\Psi$ & 文 & $I$ & $I$ & $I$ & & $I$ & & z & & 文 & 完 \\
\hline 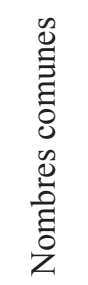 & 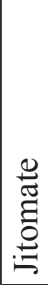 & 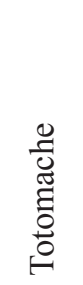 & 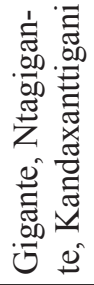 & 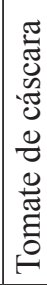 & $\begin{array}{l}0 \\
0 \\
0 \\
0 \\
0 \\
\tilde{0} \\
0 \\
0\end{array}$ & 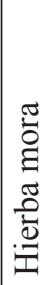 & $\begin{array}{l}\stackrel{0}{0} \\
0 \\
0 \\
0 \\
0 \\
\stackrel{0}{0} \\
.0\end{array}$ & \begin{tabular}{c}
0 \\
0 \\
0 \\
0 \\
0 \\
0 \\
0 \\
$\stackrel{0}{0}$ \\
.0 \\
\hdashline 0
\end{tabular} & & 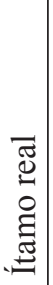 & & 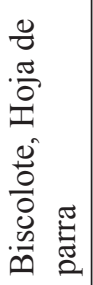 & & & 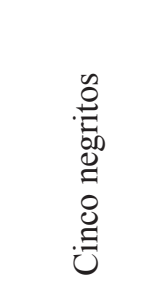 \\
\hline 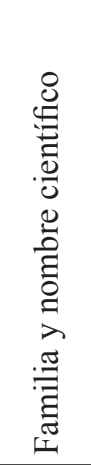 & 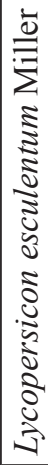 & 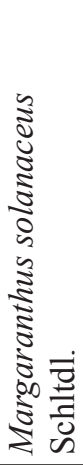 & 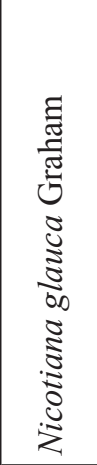 & 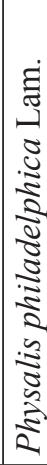 & 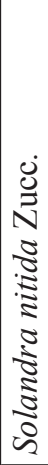 & 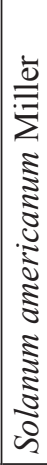 & 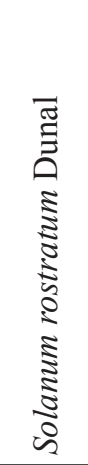 & 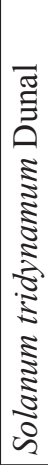 & 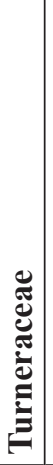 & 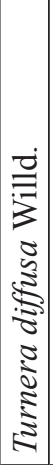 & 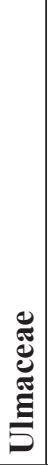 & 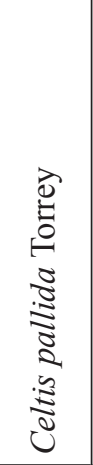 & 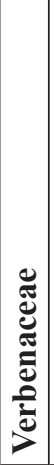 & 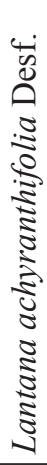 & 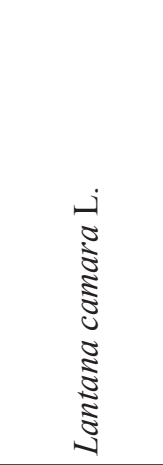 \\
\hline
\end{tabular}


Paredes-Flores et al.: Estudio etnobotánico de Zapotitlán Salinas, Puebla

\begin{tabular}{|c|c|c|c|c|c|c|}
\hline 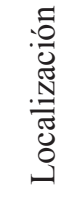 & $\sum^{0}$ & & $\underset{\Xi}{\Xi}$ & & $\underset{\pi}{\pi}$ & $\stackrel{\Xi}{\Sigma}$ \\
\hline $\begin{array}{l}\frac{.}{0} \\
\stackrel{\Xi}{E}\end{array}$ & $\bar{\sim}$ & & $\vec{U}$ & & $\bar{\sim}$ & $\begin{array}{l}0 \\
\hat{\omega}\end{array}$ \\
\hline 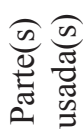 & $\begin{array}{l}\stackrel{\pi}{I} \\
I\end{array}$ & & 宝 & & 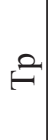 & 届 \\
\hline $\begin{array}{l}\mathscr{n} \\
\stackrel{0}{\emptyset} \\
\stackrel{0}{n}\end{array}$ & $\begin{array}{l}\stackrel{f}{r}= \\
\stackrel{-}{n}=\end{array}$ & & $N$ & & $\nabla$ & 6 \\
\hline $\begin{array}{l}\frac{\pi}{0} \\
\dot{0} \\
\dot{0}\end{array}$ & 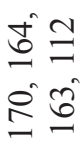 & & & & 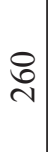 & 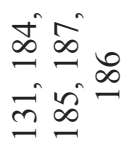 \\
\hline 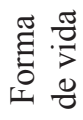 & $\dot{z}$ & & $\dot{z}$ & & $I$ & $\dot{z}$ \\
\hline 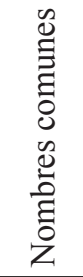 & 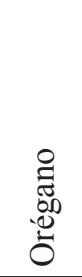 & & $\stackrel{\pi}{\square}$ & & 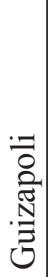 & 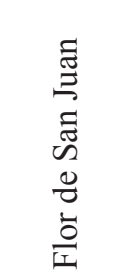 \\
\hline 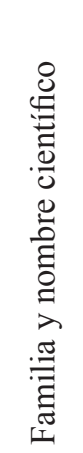 & 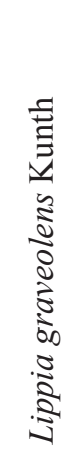 & 总 & 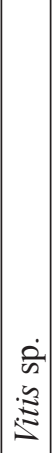 & 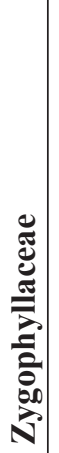 & 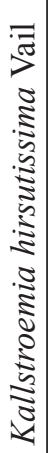 & 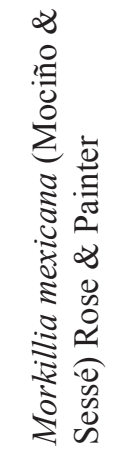 \\
\hline
\end{tabular}


Apéndice 2. Muestreos de las especies de árboles y arbustos en los tipos de vegetación del entorno de Zapotitlán Salinas, Puebla. Área $(\mathrm{A})=500 \mathrm{~m}^{2}, \mathrm{M}=$ Número de cuadros de muestreo; $\mathrm{mi}=$ número de veces que aparece en los cuadros, $\mathrm{ni}=$ Abundancia, $\mathrm{Fi}=$ Frecuencia $=\mathrm{mi} / \mathrm{M} \times 100, \mathrm{fi}=\mathrm{Fi} / \sum \mathrm{Fi}, \mathrm{Di}=$ Densidad = ni/A; Usos: Ar $=$ Artesania, $\mathrm{F}=$ Forraje, $\mathrm{Cr}=$ Cera, $\mathrm{S}=$ Sombra, $\mathrm{To}=$ Tóxico, $\mathrm{Cv}=$ Cerca viva, $\mathrm{Ba}=$ Bebida alcohólica, $\mathrm{C}=$ Combustible, $\mathrm{Co}=$ Construcción, $\mathrm{Or}=$ Ornamental, $\mathrm{A}=$ Comestible, $\mathrm{T}=$ Tinta, $\mathrm{J}=$ Juguete, $\mathrm{I}=$ Industrial, $\mathrm{M}=$ Medicinal, $\mathrm{S} / \mathrm{U}=$ Sin usos aparentes (las especies señalas con *, no están dentro del catálogo etnobotánico, porque no tienen usos).

\begin{tabular}{|c|c|c|c|c|c|c|c|c|}
\hline \multicolumn{9}{|c|}{ Mezquital } \\
\hline ESPECIE & $\mathrm{mi}$ & M & $\mathrm{Fi}$ & fi & \# usos & ni & Di & Usos \\
\hline \multicolumn{9}{|l|}{ Agavaceae } \\
\hline Agave karwinskii & 4 & 5 & 80 & 0.078 & 1 & 75 & 0.15 & $\mathrm{~A}$ \\
\hline Agave marmorata & 3 & 5 & 60 & 0.059 & 4 & 4 & 0.008 & $\mathrm{M}, \mathrm{A}, \mathrm{Ba}, \mathrm{I}$ \\
\hline \multicolumn{9}{|l|}{ Burseraceae } \\
\hline Bursera schlechtendalii & 1 & 5 & 20 & 0.020 & 1 & 4 & 0.008 & M \\
\hline \multicolumn{9}{|l|}{ Cactaceae } \\
\hline Ferocactus robustus & 1 & 5 & 20 & 0.020 & 1 & 5 & 0.01 & $\mathrm{~F}$ \\
\hline $\begin{array}{l}\text { Myrtillocactus } \\
\text { geometrizans }\end{array}$ & 4 & 5 & 80 & 0.078 & 5 & 24 & 0.048 & $\mathrm{~A}, \mathrm{~F}, \mathrm{Cv}, \mathrm{C}, \mathrm{Ba}$ \\
\hline Opuntia pilifera & 4 & 5 & 80 & 0.078 & 1 & 5 & 0.01 & A \\
\hline *Opuntia pubescens & 3 & 5 & 60 & 0.059 & 0 & 2 & 0.004 & $\mathrm{~S} / \mathrm{U}$ \\
\hline Pachycereus hollianus & 5 & 5 & 100 & 0.098 & 3 & 30 & 0.06 & $\mathrm{~A}, \mathrm{Co}, \mathrm{Cv}, \mathrm{C}$ \\
\hline Stenocereus stellatus & 2 & 5 & 40 & 0.039 & 4 & 12 & 0.024 & $\mathrm{~A}, \mathrm{~F}, \mathrm{Cv}, \mathrm{C}$ \\
\hline \multicolumn{9}{|l|}{ Caesalpiniaceae } \\
\hline Parkinsonia praecox & 3 & 5 & 60 & 0.059 & 3 & 7 & 0.014 & $\mathrm{~F}, \mathrm{C}, \mathrm{M}$ \\
\hline \multicolumn{9}{|l|}{ Mimosaceae } \\
\hline Prosopis laevigata & 5 & 5 & 100 & 0.098 & 3 & 30 & 0.06 & $\mathrm{~F}, \mathrm{C}, \mathrm{Co}$ \\
\hline \multicolumn{9}{|l|}{ Simaroubaceae } \\
\hline $\begin{array}{l}\text { Castela erecta subsp. } \\
\quad \text { texana }\end{array}$ & 5 & 5 & 100 & 0.098 & 1 & 29 & 0.058 & $\mathrm{M}$ \\
\hline \multicolumn{9}{|l|}{ Rutaceae } \\
\hline $\begin{array}{l}\text { *Zanthoxylum } \\
\quad \text { liebmannianum }\end{array}$ & 2 & 5 & 40 & 0.039 & 0 & 10 & 0.02 & $\mathrm{~S} / \mathrm{U}$ \\
\hline \multicolumn{9}{|l|}{ Ulmaceae } \\
\hline Celtis pallida & 5 & 5 & 100 & 0.098 & 1 & 22 & 0.044 & $\mathrm{M}$ \\
\hline
\end{tabular}


Apéndice 2. Continuación.

\begin{tabular}{|c|c|c|c|c|c|c|c|c|}
\hline \multicolumn{9}{|c|}{ Tetechera-Candelillar } \\
\hline ESPECIE & $\mathrm{mi}$ & M & $\mathrm{Fi}$ & fi & \# usos & ni & Di & Usos \\
\hline \multicolumn{9}{|l|}{ Cactaceae } \\
\hline $\begin{array}{l}\text { Cephalocereus } \\
\text { columna-trajani }\end{array}$ & 2 & 5 & 40 & 0.063 & 1 & 8 & 0.016 & $\mathrm{C}$ \\
\hline $\begin{array}{l}\text { Ferocactus latispinus } \\
\quad \text { var. spiralis }\end{array}$ & 1 & 5 & 20 & 0.031 & 2 & 6 & 0.012 & $\mathrm{~F}, \mathrm{~A}, \mathrm{Or}$ \\
\hline Neobuxbaumia tetetzo & 2 & 5 & 40 & 0.063 & 4 & 32 & 0.064 & $\mathrm{C}, \mathrm{F}, \mathrm{A}$ \\
\hline Pachycereus hollianus & 5 & 5 & 100 & 0.156 & 3 & 34 & 0.068 & $\mathrm{~A}, \mathrm{Co}, \mathrm{Cv}, \mathrm{C}$ \\
\hline \multicolumn{9}{|l|}{ Caesalpiniaceae } \\
\hline Parkinsonia praecox & 5 & 5 & 100 & 0.156 & 3 & 10 & 0.02 & $\mathrm{~F}, \mathrm{C}, \mathrm{M}$ \\
\hline \multicolumn{9}{|l|}{ Mimosaceae } \\
\hline Mimosa luisana & 4 & 5 & 80 & 0.125 & 2 & 20 & 0.04 & $\mathrm{C}, \mathrm{F}$ \\
\hline Prosopis laevigata & 5 & 5 & 100 & 0.156 & 3 & 8 & 0.016 & $\mathrm{~F}, \mathrm{C}, \mathrm{Co}$ \\
\hline \multicolumn{9}{|l|}{ Nolinaceae } \\
\hline Beaucarnea gracilis & 1 & 5 & 20 & 0.031 & 1 & 9 & 0.018 & Or \\
\hline \multicolumn{9}{|l|}{ Simaroubaceae } \\
\hline $\begin{array}{l}\text { Castela erecta subsp. } \\
\text { texana }\end{array}$ & 5 & 5 & 100 & 0.156 & 1 & 24 & 0.048 & $\mathrm{M}$ \\
\hline \multicolumn{9}{|l|}{ Rhamnaceae } \\
\hline Ziziphus amole & 1 & 5 & 20 & 0.031 & 1 & 1 & 0.002 & M \\
\hline \multicolumn{9}{|c|}{ Cardonal } \\
\hline ESPECIE & $\mathrm{mi}$ & $\mathrm{M}$ & $\mathrm{Fi}$ & fi & \# usos & ni & $\mathrm{Di}$ & Usos \\
\hline \multicolumn{9}{|l|}{ Burseraceae } \\
\hline $\begin{array}{l}\text { Bursera schlechtendalii } \\
\text { Cactaceae }\end{array}$ & \multicolumn{7}{|c|}{ Cactaceae } & M \\
\hline $\begin{array}{l}\text { Cephalocereus } \\
\text { columna-trajani }\end{array}$ & 2 & 5 & 40 & 0.069 & 1 & 19 & 0.038 & $\mathrm{C}$ \\
\hline Neobuxbaumia tetetzo & 2 & 5 & 40 & 0.069 & 4 & 8 & 0.016 & $\mathrm{~A}, \mathrm{~F}, \mathrm{C}$ \\
\hline Pachycereus hollianus & 5 & 5 & 100 & 0.172 & 3 & 20 & 0.04 & $\mathrm{~A}, \mathrm{C}, \mathrm{Co}, \mathrm{Cv}$ \\
\hline Peniocereus viperinus & 1 & 5 & 20 & 0.034 & 2 & 1 & 0.002 & Or, A \\
\hline \multicolumn{9}{|l|}{ Caesalpiniaceae } \\
\hline $\begin{array}{l}\text { Caesalpinia } \\
\text { melanadenia }\end{array}$ & 1 & 5 & 20 & 0.034 & 1 & 10 & 0.02 & $\mathrm{~F}$ \\
\hline Parkinsonia praecox & 5 & 5 & 100 & 0.172 & 3 & 23 & 0.046 & $\mathrm{~F}, \mathrm{C}, \mathrm{M}$ \\
\hline
\end{tabular}


Apéndice 2. Continuación.

Mimosaceae

Prosopis laevigata

$$
\begin{array}{llllllll}
5 & 5 & 100 & 0.172 & 3 & 24 & 0.048 & \text { F, C, Co }
\end{array}
$$

Zapoteca formosa

$\begin{array}{llllllll}1 & 5 & 20 & 0.034 & 2 & 3 & 0.006 & \text { F,J }\end{array}$

Simaroubaceae

Castela erecta subsp. texana

$$
\begin{array}{llllllll}
5 & 5 & 100 & 0.172 & 1 & 21 & 0.042 & \mathrm{~A}, \mathrm{Co}
\end{array}
$$

Matorral Espinoso

ESPECIE

mi M Fi fi \# usos ni Di Usos

Boraginaceae

Cordia curassavica

$\begin{array}{llllllll}2 & 5 & 40 & 0.040 & 1 & 2 & 0.004 & \mathrm{M}\end{array}$

Burseraceae

$\begin{array}{lllllllll}\text { Bursera schlechtendalii } & 3 & 5 & 60 & 0.060 & 1 & 3 & 0.006 & \mathrm{M}\end{array}$

Cactaceae

Myrtillocactus

geometrizans

Stenocereus stellatus

Opuntia pilifera

Pachycereus hollianus

2

54

$40 \quad 0.040$

5

$4 \quad 0.008$

$\mathrm{A}, \mathrm{F}, \mathrm{Cv}, \mathrm{C}$,

5

$0.020+4$

$\mathrm{Ba}$

Pachycereus marginatus

$\begin{array}{cccccccc}1 & 5 & 20 & 0.020 & 4 & 3 & 0.006 & \mathrm{~A}, \mathrm{~F}, \mathrm{Cv}, \mathrm{C} \\ 4 & 5 & 80 & 0.080 & 1 & 3 & 0.006 & \mathrm{~A} \\ 5 & 5 & 100 & 0.100 & 3 & 5 & 0.01 & \mathrm{~A}, \mathrm{Cv}, \mathrm{C}, \mathrm{Co} \\ 3 & 5 & 60 & 0.060 & 2 & 4 & 0.008 & \mathrm{~F}, \mathrm{C}, \mathrm{Cv}, \mathrm{M}\end{array}$

Caesalpiniaceae

Parkinsonia praecox

$$
\begin{array}{llllllll}
5 & 5 & 100 & 0.100 & 3 & 5 & 0.01 & \text { F, C, M }
\end{array}
$$

Convolvulaceae

Ipomoea pauciflora

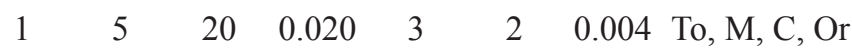

Fabaceae

*Dalea carthagenensis

1

$5 \quad 20$

$20 \quad 0.020 \quad 0$

$49 \quad 0.098$

$\mathrm{S} / \mathrm{U}$

Mimosaceae

Acacia farnesiana

Acacia constricta

Prosopis laevigata

254

40

$$
0.0402
$$

30.006

F, C, M

25

40

4

80

0.040

$5 \quad 0.01$

$\mathrm{F}$

Simaroubaceae

Castela erecta subsp. texana

2

5

$$
40
$$

$40 \quad 0.040 \quad 2$

30.006

M

Ulmaceae

Celtis pallida

4

$80 \quad 0.0$

$\begin{array}{llll}0.080 & 1 & 8 & 0.016\end{array}$

M 
Apéndice 2. Continuación.

\begin{tabular}{|c|c|c|c|c|c|c|c|c|c|}
\hline \multicolumn{10}{|l|}{ Verbenaceae } \\
\hline Lantana achyranthifolia & 1 & 5 & 20 & 0.020 & \multicolumn{2}{|l|}{0} & \multicolumn{2}{|c|}{0.014} & M \\
\hline Lantana camara & 3 & 5 & 60 & \multicolumn{2}{|c|}{0.060} & & \multicolumn{2}{|c|}{0.008} & $\mathrm{M}, \mathrm{A}$ \\
\hline \multicolumn{10}{|l|}{ Zygophyllaceae } \\
\hline Morkillia mexicana & 2 & 5 & 40 & \multicolumn{2}{|c|}{0.040} & & \multicolumn{2}{|c|}{0.004} & Or \\
\hline \multicolumn{10}{|c|}{ Izotal } \\
\hline ESPECIE & $\mathrm{mi}$ & M & $\mathrm{Fi}$ & fi & \# usos & ni & A & Di & Usos \\
\hline \multicolumn{10}{|l|}{ Agavaceae } \\
\hline Yucca periculosa & 3 & 5 & 60 & 0.097 & 1 & 16 & 500 & 0.032 & A \\
\hline \multicolumn{10}{|l|}{ Cactaceae } \\
\hline Neouxbaumia tetetzo & 1 & 5 & 20 & 0.032 & 4 & 1 & 500 & 0.002 & $\mathrm{~A}, \mathrm{~F}, \mathrm{C}$ \\
\hline Opuntia pilifera & 4 & 5 & 80 & 0.129 & 1 & 4 & 500 & 0.008 & A \\
\hline \multicolumn{10}{|l|}{ Caesalpiniaceae } \\
\hline Parkinsonia praecox & 5 & 5 & 100 & 0.161 & 3 & 5 & 500 & 0.01 & $\mathrm{~F}, \mathrm{C}, \mathrm{M}$ \\
\hline \multicolumn{10}{|l|}{ Convolvulaceae } \\
\hline Ipomoea pauciflora & 3 & 5 & 60 & 0.097 & 3 & 3 & 500 & 0.006 & $\begin{array}{c}\text { To, } \mathrm{M}, \mathrm{C}, \\
\text { Or }\end{array}$ \\
\hline \multicolumn{10}{|l|}{ Lamiaceae } \\
\hline *No determinada 1 & 5 & 5 & 100 & 0.161 & 0 & 50 & 500 & 0.1 & $\mathrm{~S} / \mathrm{U}$ \\
\hline \multicolumn{10}{|l|}{ Mimosaceae } \\
\hline Acacia constricta & 3 & 5 & 60 & 0.097 & 1 & 3 & 500 & 0.006 & $\mathrm{~F}$ \\
\hline Acacia farnesiana & 2 & 5 & 40 & 0.065 & 2 & 3 & 500 & 0.006 & $\mathrm{~F}, \mathrm{C}, \mathrm{M}$ \\
\hline Acacia subungulata & 3 & 5 & 60 & 0.097 & 1 & 7 & 500 & 0.014 & $\mathrm{~F}$ \\
\hline \multicolumn{10}{|l|}{ Rhamnaceae } \\
\hline $\begin{array}{l}\text { Karwinskia } \\
\quad \text { humboldtiana }\end{array}$ & 2 & 5 & 40 & 0.065 & 1 & 4 & 500 & 0.008 & Or \\
\hline
\end{tabular}

ESPECIE $\quad \mathrm{mi} M \quad \mathrm{Fi}$ fi \# usos ni Di Usos

\section{Anacardiaceae}

*Rhus sp.

$\begin{array}{llllllll}2 & 5 & 40 & 0.083 & 0 & 2 & 0.004 & \mathrm{~S} / \mathrm{U}\end{array}$

\section{Berberidaceae}

*Berberis sp.

$\begin{array}{llllllll}2 & 5 & 40 & 0.083 & 0 & 5 & 0.01 & \mathrm{~S} / \mathrm{U}\end{array}$

Cactaceae

Opuntia sp.

$\begin{array}{lllllll}2 & 5 & 40 & 0.083 & 1 & 2 & 0.004\end{array}$


Apéndice 2. Continuación.

\section{Fagaceae}

Quercus sp.

Nolinaceae

$\begin{array}{llllllllll}\text { Dasylirion serratifolium } & 4 & 5 & 40 & 0.083 & 2 & 3 & 0.006 & \text { C, A }\end{array}$

\section{Agavaceae}

Yucca periculosa

$\begin{array}{lllllll}3 & 5 & 60 & 0.125 & 1 & 4 & 0.008\end{array}$

A

Arecaceae

Brahea dulcis

$\begin{array}{lllll}3 & 5 & 60 & 0.125 & 2\end{array}$

49

$0.098 \mathrm{M}, \mathrm{Or}, \mathrm{Ar}$

\section{Rosaceae}

$\begin{array}{llllllllll}\text { Malacomeles denticulata } & 5 & 5 & 100 & 0.208 & 1 & 40 & 0.08 & \text { F }\end{array}$

Familia No Det.

\begin{tabular}{|c|c|c|c|c|c|c|c|c|}
\hline *Especie no det. & 3 & 5 & 60 & 0.125 & 1 & 9 & 0.018 & $\mathrm{~S} / \mathrm{U}$ \\
\hline \multicolumn{9}{|c|}{ Tetechera-Candelillar } \\
\hline ESPECIE & $\mathrm{mi}$ & M & $\mathrm{Fi}$ & fi & \# usos & ni & $\mathrm{Di}$ & Usos \\
\hline \multicolumn{9}{|l|}{ Agavaceae } \\
\hline Agave karwinskii & 1 & 5 & 20 & 0.036 & 1 & 8 & 0.016 & $\mathrm{~A}$ \\
\hline \multicolumn{9}{|l|}{ Apocynaceae } \\
\hline Plumeria rubra & 1 & 5 & 20 & 0.036 & 1 & 18 & 0.036 & Or \\
\hline \multicolumn{9}{|l|}{ Cactaceae } \\
\hline *Coryphantha pallida & 1 & 5 & 20 & 0.036 & 0 & 11 & 0.022 & $\mathrm{~S} / \mathrm{U}$ \\
\hline $\begin{array}{l}\text { Echinocactus } \\
\text { platyacanthus }\end{array}$ & 1 & 5 & 20 & 0.036 & 2 & 9 & 0.018 & $\mathrm{~F}$ \\
\hline Mammillaria sphacelata & 2 & 5 & 40 & 0.071 & 1 & 19 & 0.038 & Or \\
\hline Neobuxbaumia tetetzo & 1 & 5 & 20 & 0.036 & 4 & 32 & 0.064 & $\mathrm{C}, \mathrm{F}, \mathrm{A}$ \\
\hline Opuntia pilifera & 4 & 5 & 80 & 0.143 & 1 & 10 & 0.02 & $\mathrm{~A}$ \\
\hline \multicolumn{9}{|l|}{ Caesalpiniaceae } \\
\hline Parkinsonia praecox & 3 & 5 & 60 & 0.107 & 2 & 18 & 0.036 & $\mathrm{~F}, \mathrm{C}, \mathrm{M}$ \\
\hline \multicolumn{9}{|l|}{ Celastraceae } \\
\hline *Maytenus phyllantoides & 2 & 5 & 40 & 0.071 & 3 & 2 & 0.004 & $\mathrm{~S} / \mathrm{U}$ \\
\hline \multicolumn{9}{|l|}{ Euphorbiaceae } \\
\hline Euphorbia antisyphilitica & 1 & 5 & 20 & 0.036 & 0 & 35 & 0.07 & $\mathrm{Cr}$ \\
\hline Jatropha dioica & 1 & 5 & 20 & 0.036 & 1 & 2 & 0.004 & M \\
\hline *Manihotoides pauciflora & 1 & 5 & 20 & 0.036 & 1 & 2 & 0.004 & $\mathrm{~S} / \mathrm{U}$ \\
\hline
\end{tabular}


Apéndice 2. Continuación.

Mimosaceae

Mimosa luisana

2

540

$\begin{array}{lll}40 & 0.071 \quad 2\end{array}$

$15 \quad 0.03$

$\mathrm{C}, \mathrm{F}$

Prosopis laevigata

4

5

$\begin{array}{lll}80 & 0.143 \quad 3\end{array}$

$7 \quad 0.014$

F, C, Co

Simaroubaceae

Castela erecta subsp. texana

Zygophyllaceae

Morkillia mexicana

15

$20 \quad 0.036$

$9 \quad 0.018$

Or 